\title{
1 List of Figures
}

Figure A1: $\mathrm{O}_{3}$ trends of monthly mean characterised by loess regression...................3

Figure A2: Seasonal cycles of $\mathrm{O}_{3}$ (ppbv) for the period 1996-2005 inclusive...............11

Figure B1: Loess trends of CHIMERE simulated monthly mean $\mathrm{O}_{3} \ldots \ldots \ldots \ldots \ldots \ldots \ldots \ldots . . . \ldots \ldots$

\section{List of Tables}

Table A1: Characterisation and categorisation of $\mathrm{O}_{3}$ time series by loess................19

Table A2: Quantification of trends in $\mathrm{O}_{3}$ monthly mean 1996-2005......................20

Table A3: Quantification of trends in $\mathrm{O}_{3}$ monthly $5^{\text {th }}$ percentiles $1996-2005 \ldots \ldots \ldots \ldots . .24$

Table A4: Quantification of trends in $\mathrm{O}_{3}$ monthly $95^{\text {th }}$ percentiles $1996-2005 \ldots \ldots \ldots . .28$

Table A5: Quantification of trends in seasonal mean $\mathrm{O}_{3}$ 1996-2005......................32

Table A6: Quantification of trends in seasonal $\mathrm{O}_{3} 5^{\text {th }}$ percentiles 1996-2005............36

Table A7: Quantification of trends in seasonal $\mathrm{O}_{3}$ 95 ${ }^{\text {th }}$ percentiles $1996-2005 \ldots \ldots \ldots . . .40$

Table B1: Quantification of annual CHIMERE model trends in mean $\mathrm{O}_{3}$ 1996-2005..53

Table B2: Quantification of annual CHIMERE model trends in $\mathrm{O}_{3} 5^{\text {th }}$ percentiles 1996-

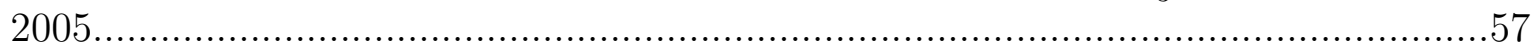

Table B3: Quantification of annual CHIMERE model trends in $\mathrm{O}_{3} 95^{\text {th }}$ percentiles

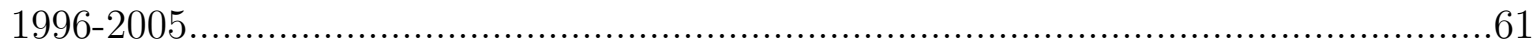

Table C1: Sites used in this study and their categorisation...............................66 


\section{Observed annual and seasonal trends}

A compilation of loess trends of monthly mean $\mathrm{O}_{3}$ for all stations in this study are given in Figure ?? and Table ?? in addition to annual $5^{\text {th }}$ and $95^{\text {th }}$ percentiles of $\mathrm{O}_{3}$. $75 \%$ coverage of monthly data was required to calculate statistics.

Trend tables summarising trends in annual mean, $5^{\text {th }}$ and $95^{\text {th }}$ percentiles for 158 European measurement sites are give in Tables ?? - ??, respectively. A compilation of seasonal cycles from daily mean, $5^{\text {th }}$, and $95^{\text {th }}$ percentiles of $\mathrm{O}_{3}$ for all stations in this study are given in Figure ??. Trend tables summarising trends in seasonal (winter $[\mathrm{DJF}]$, spring $[\mathrm{MAM}]$, summer [JJA] and autumn $[\mathrm{SON}])$ mean, $5^{\text {th }}$ and $95^{\text {th }}$ percentiles for 158 European measurement sites are give in Tables ?? - ??, respectively. 

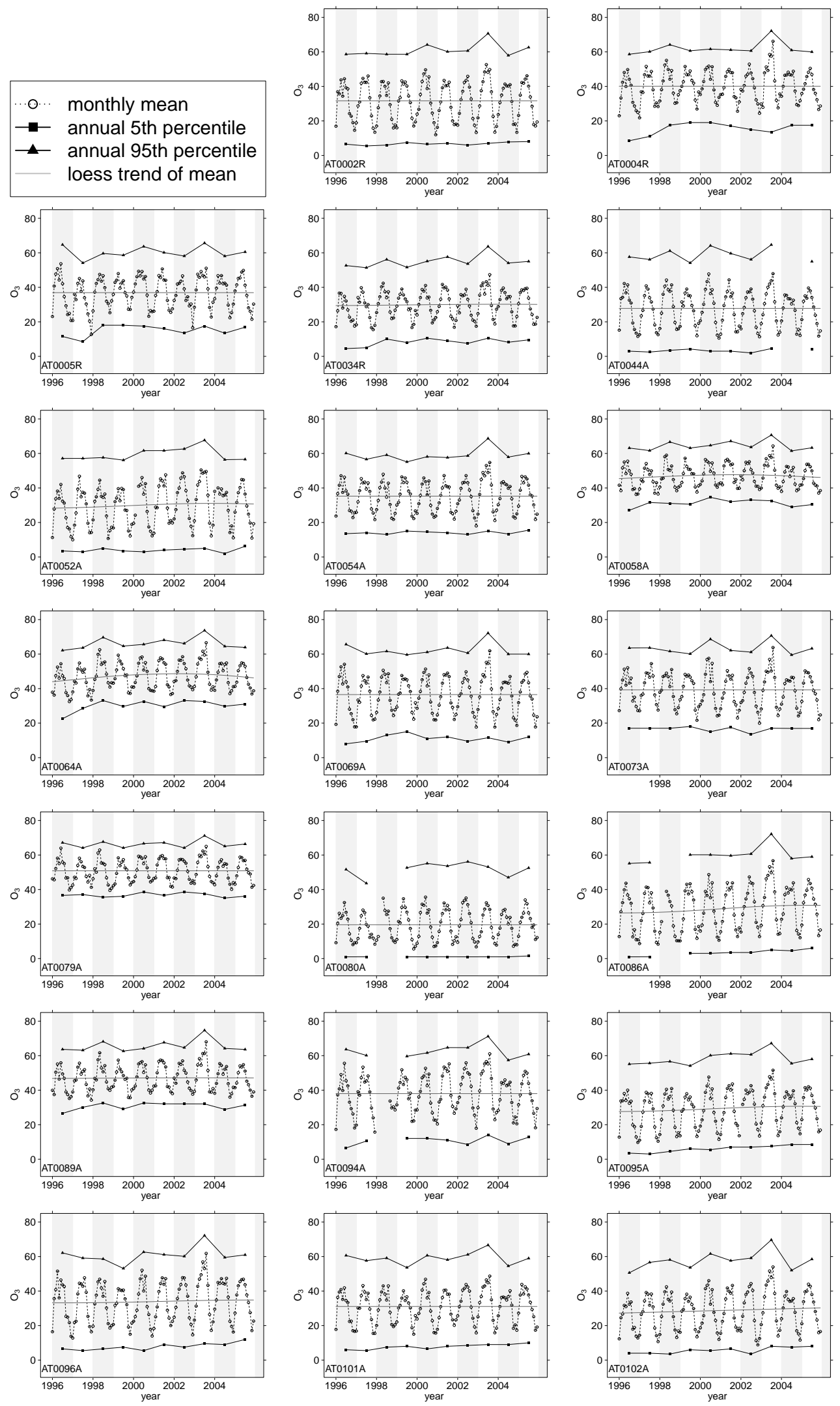

Figure $\mathrm{A} 1: \mathrm{O}_{3}$ trends of monthly mean characterised by loess regression 

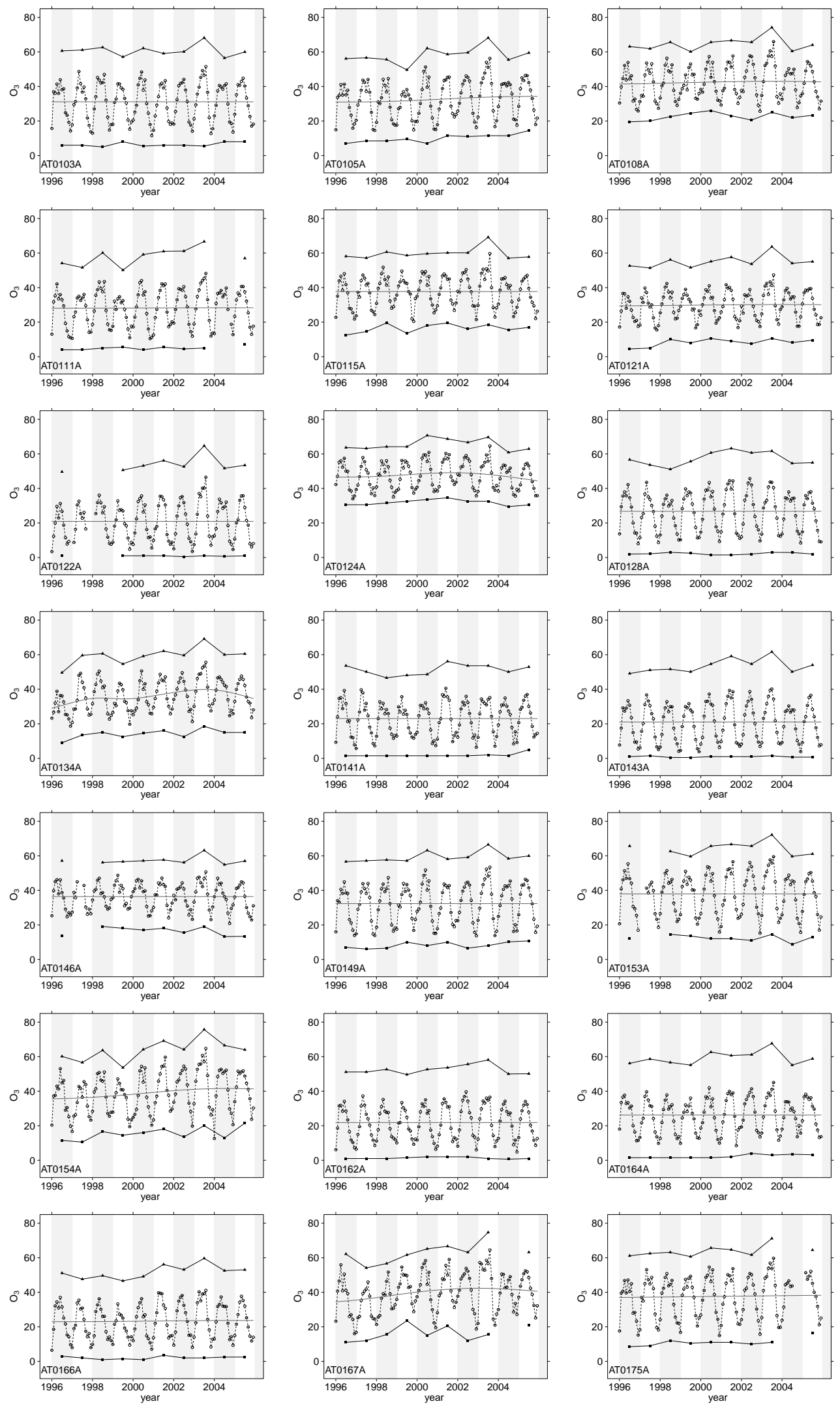

Figure A1: continued. $\mathrm{O}_{3}$ trends of monthly mean characterised by loess regression 

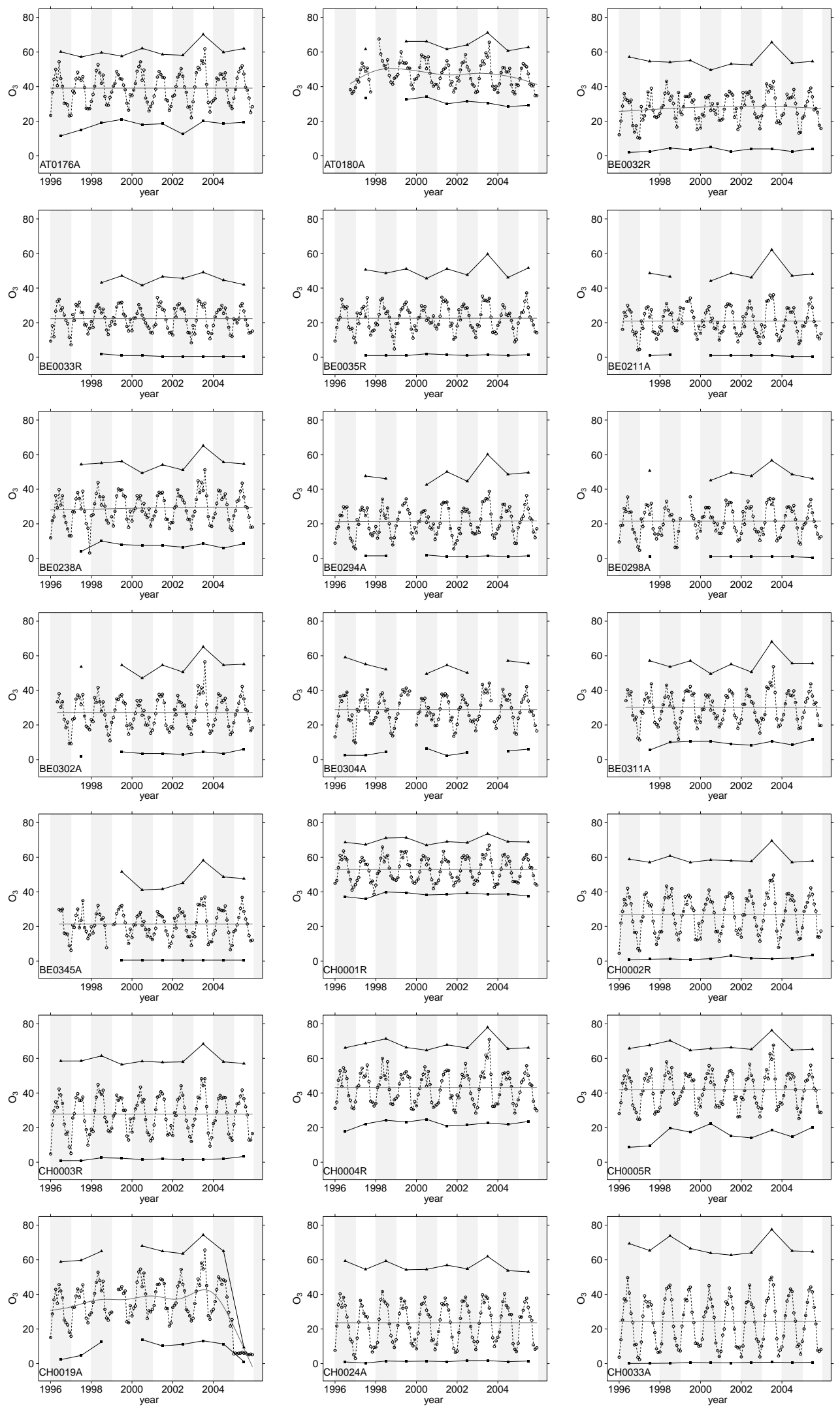

Figure A1: continued. $\mathrm{O}_{3}$ trends of monthly mean characterised by loess regression 

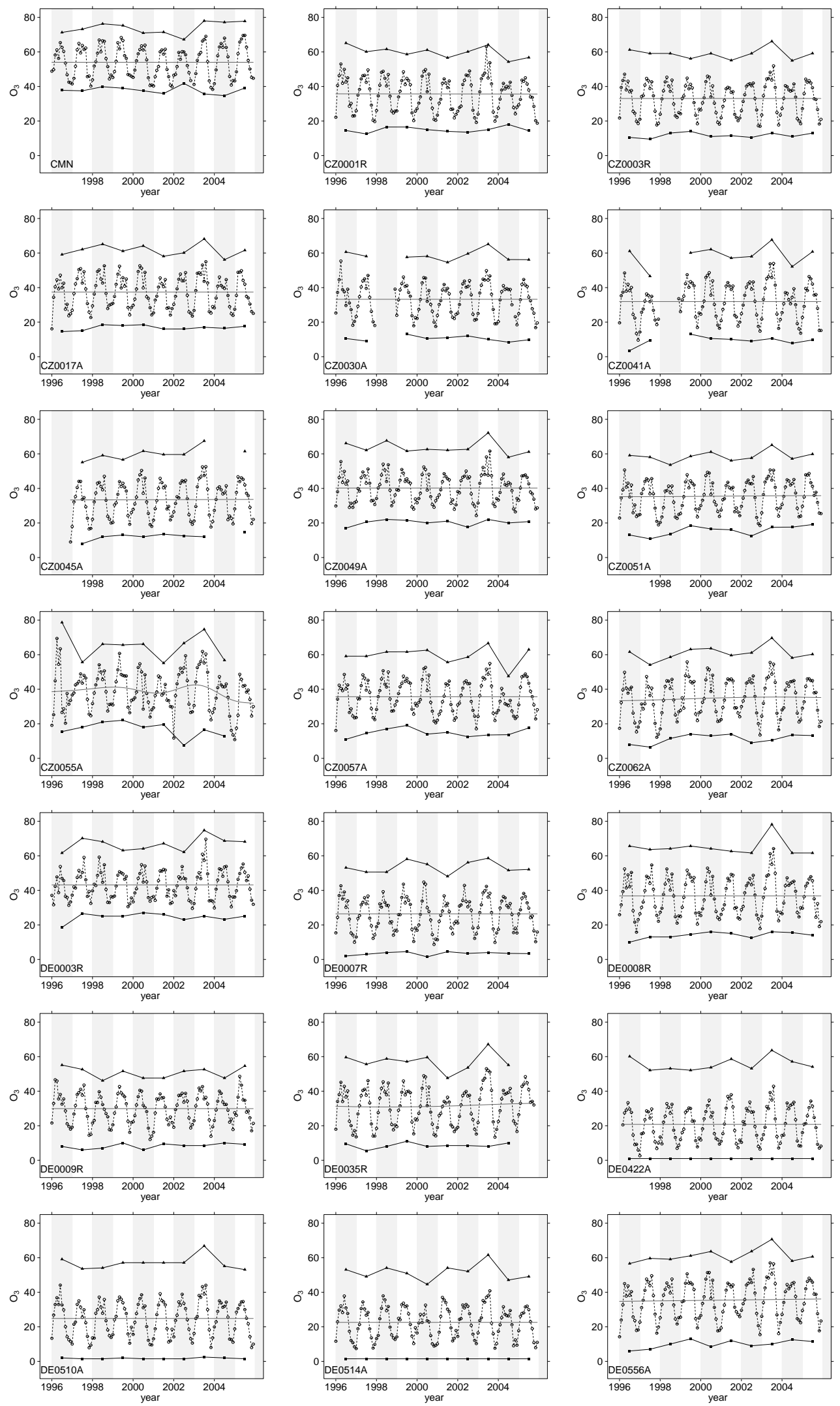

Figure A1: continued. $\mathrm{O}_{3}$ trends of monthly mean characterised by loess regression 

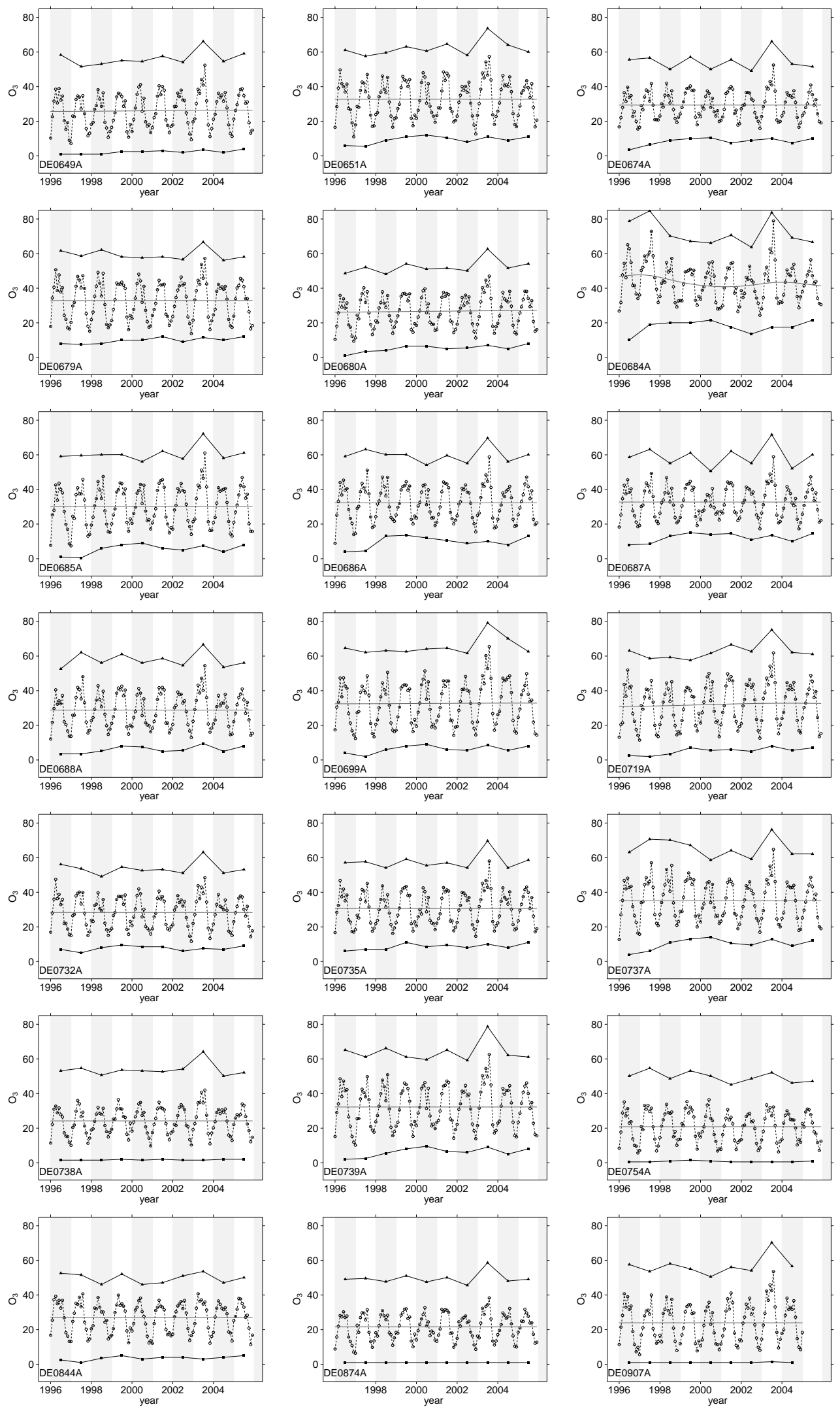

Figure A1: continued. $\mathrm{O}_{3}$ trends of monthly mean characterised by loess regression 

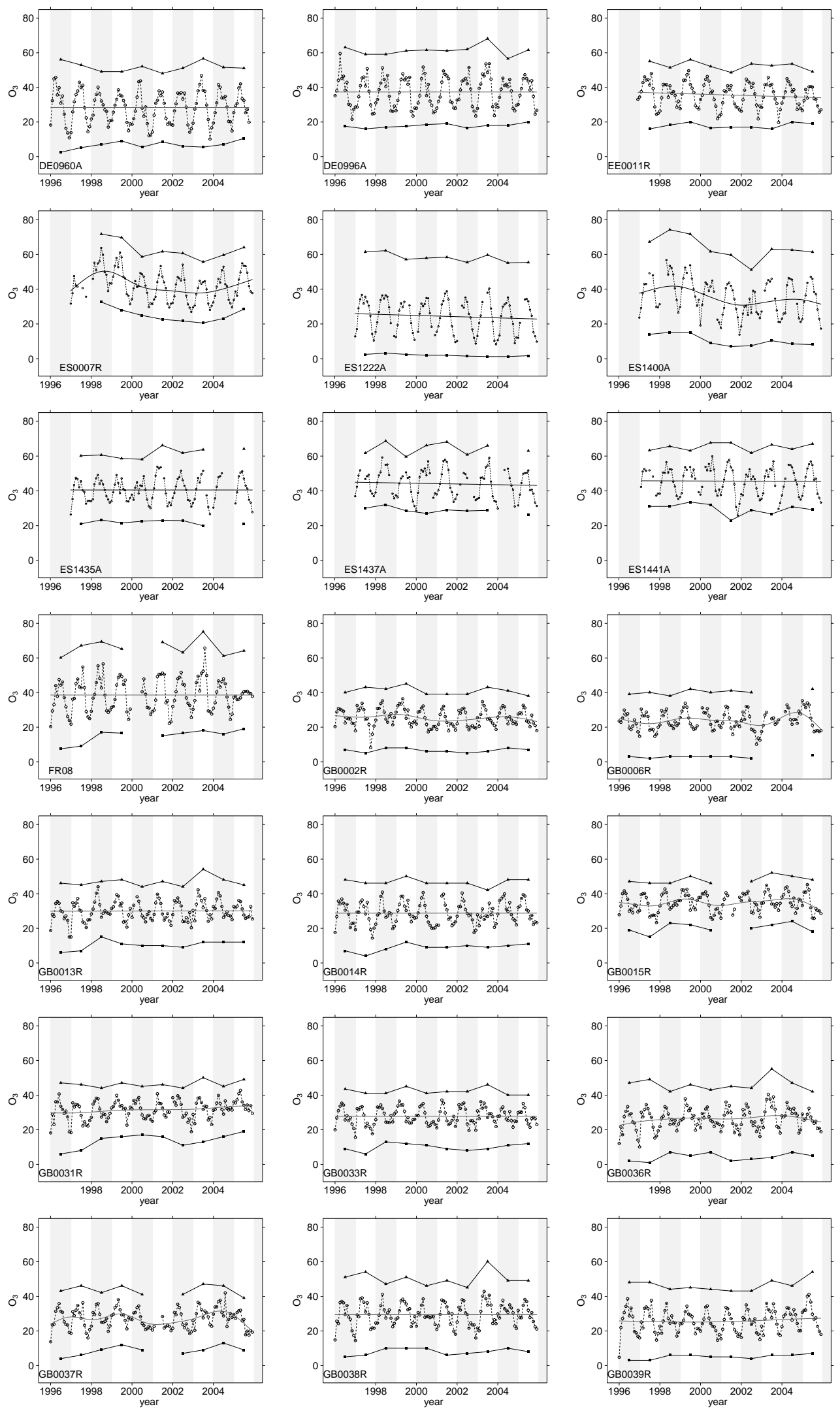

Figure A1: continued. $\mathrm{O}_{3}$ trends of monthly mean characterised by loess regression 

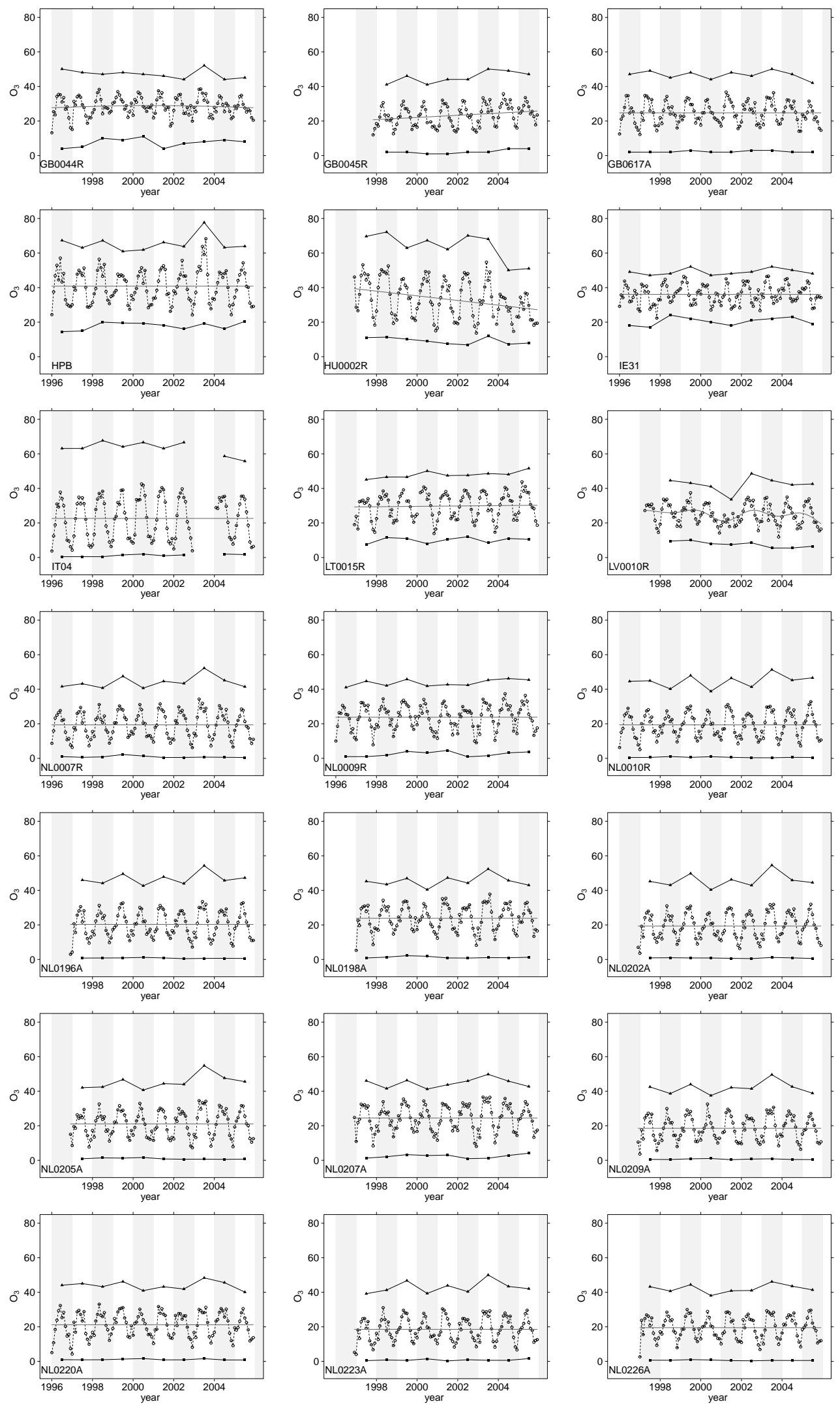

Figure A1: continued. $\mathrm{O}_{3}$ trends of monthly mean characterised by loess regression 

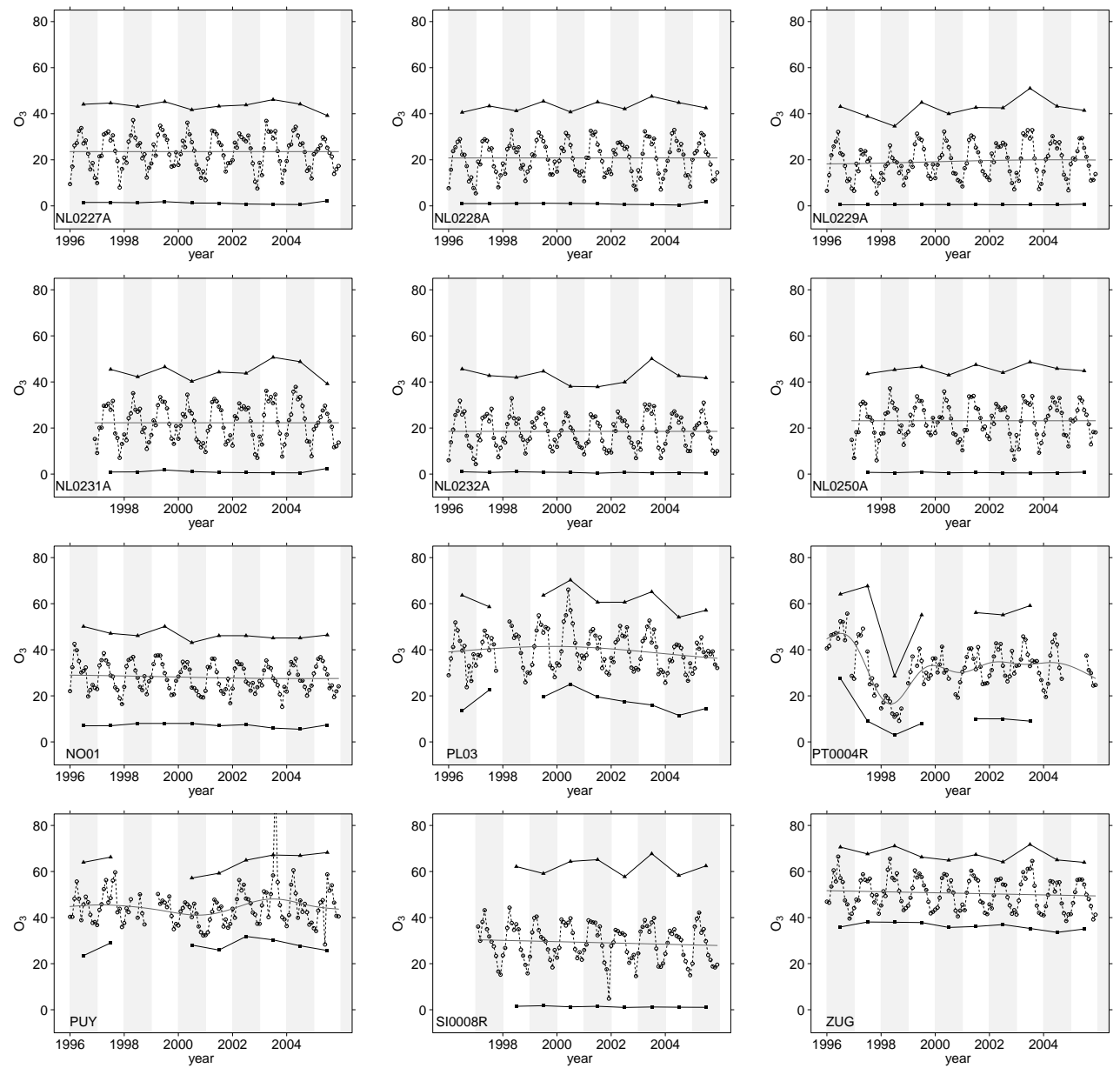

Figure A1: continued. $\mathrm{O}_{3}$ trends of monthly mean characterised by loess regression 

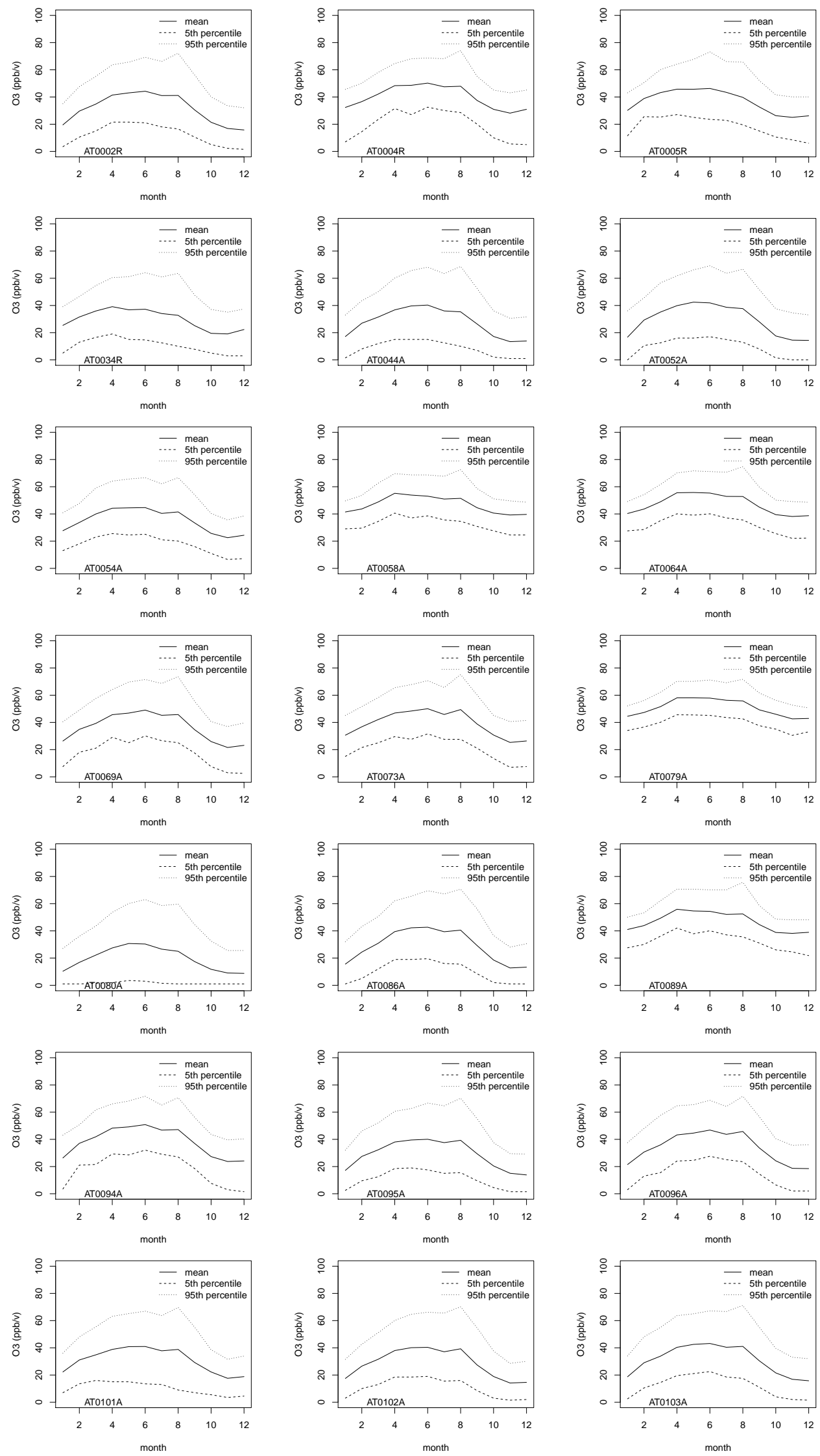

Figure A2: Seasonal cycles of $\mathrm{O}_{3}$ (ppbv) for the period 1996-2005 inclusive 

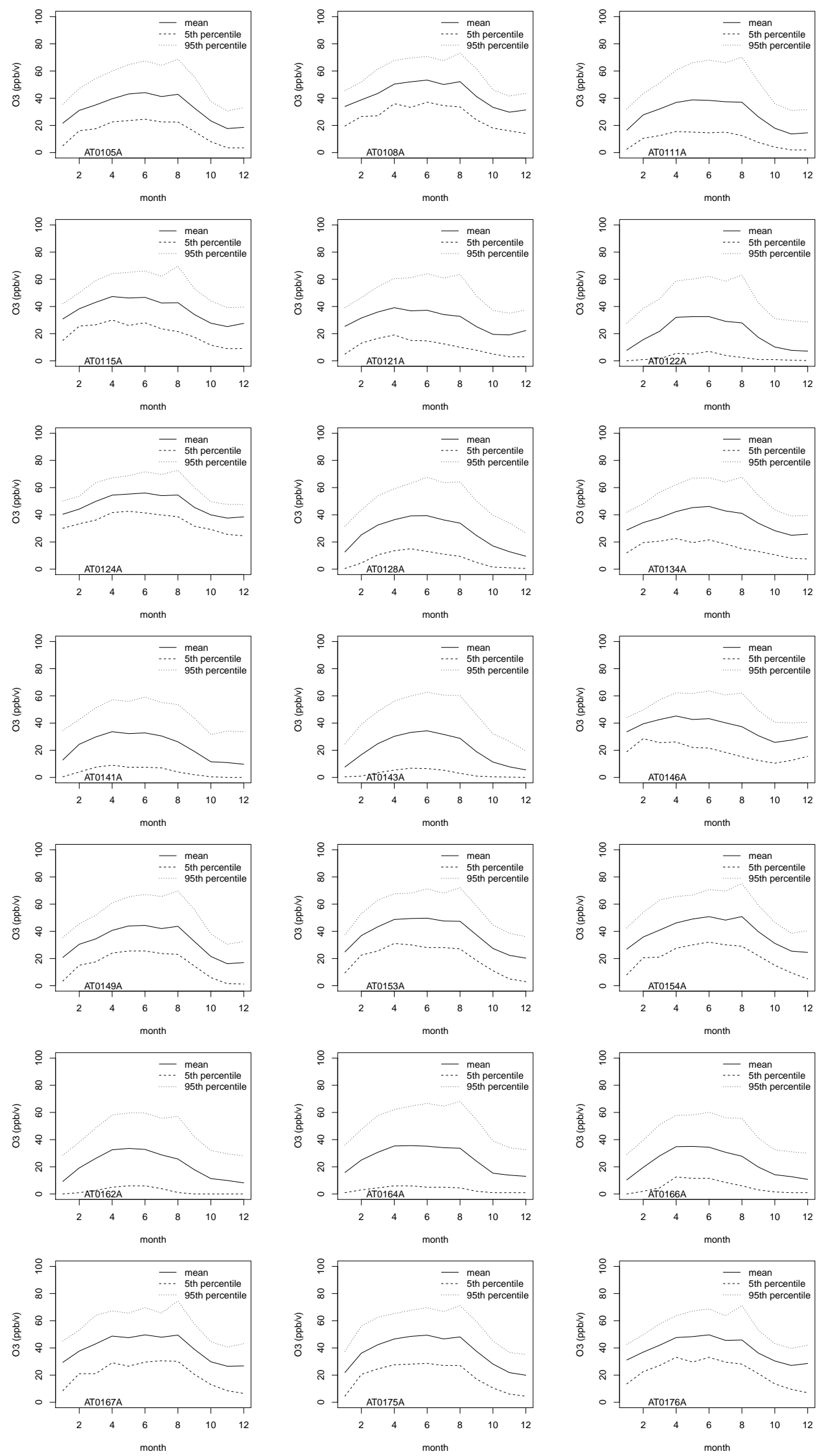

Figure A2: continued. Seasonal cycles of $\mathrm{O}_{3}$ (ppbv) for the period 1996-2005 inclusive 

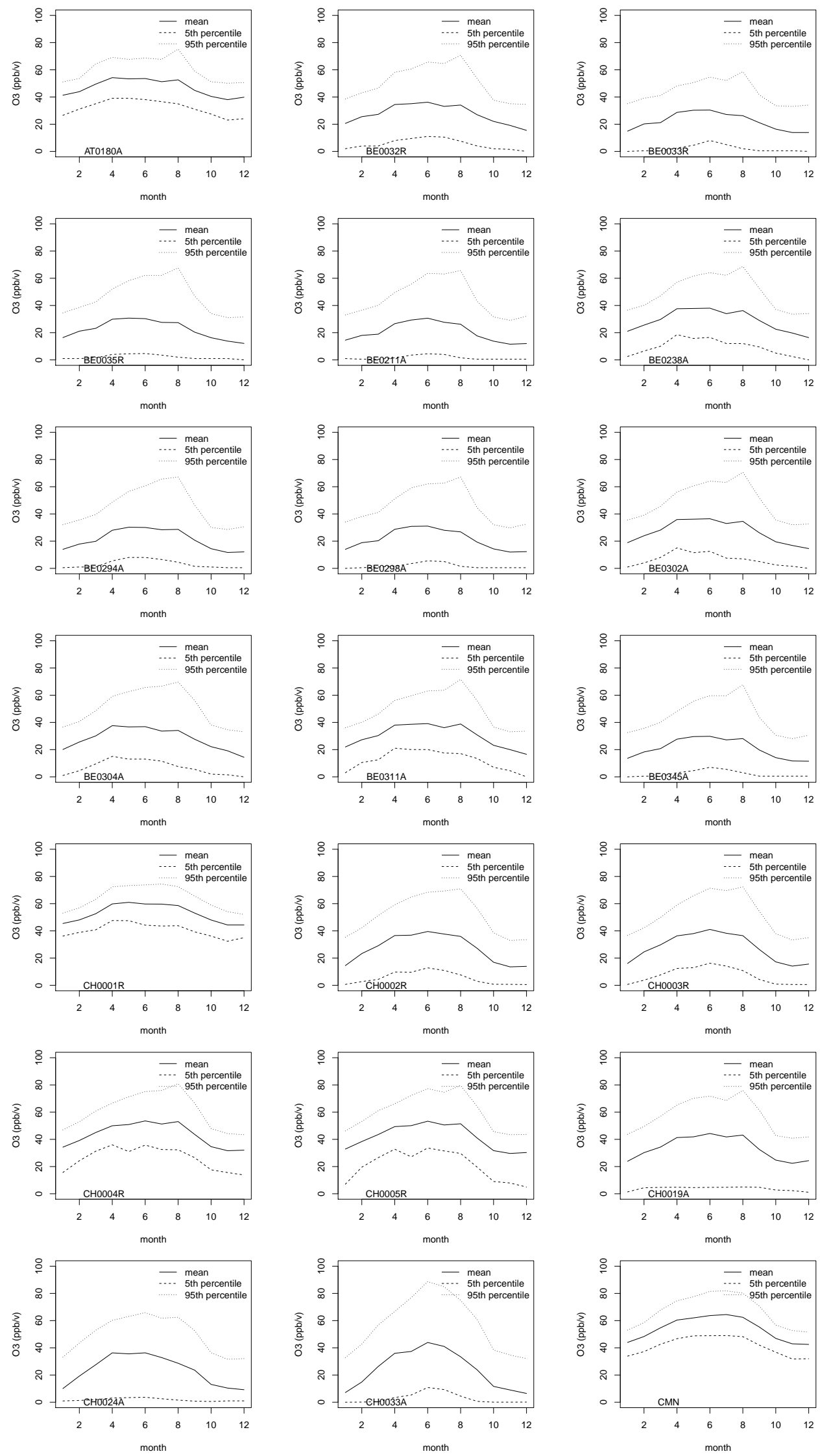

Figure A2: continued. Seasonal cycles of $\mathrm{O}_{3}$ (ppbv) for the period 1996-2005 inclusive 

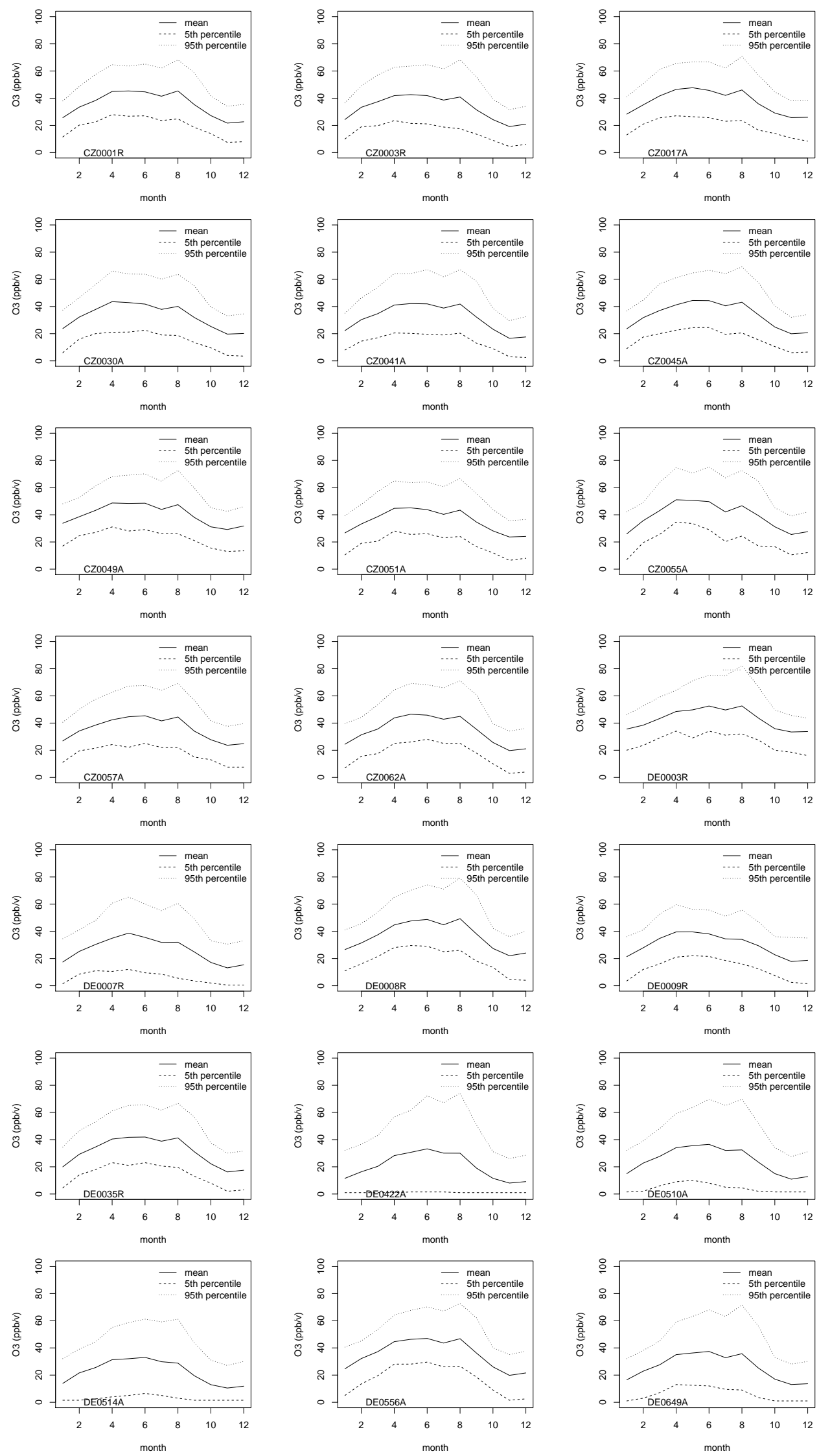

Figure A2: continued. Seasonal cycles of $\mathrm{O}_{3}$ (ppbv) for the period 1996-2005 inclusive 

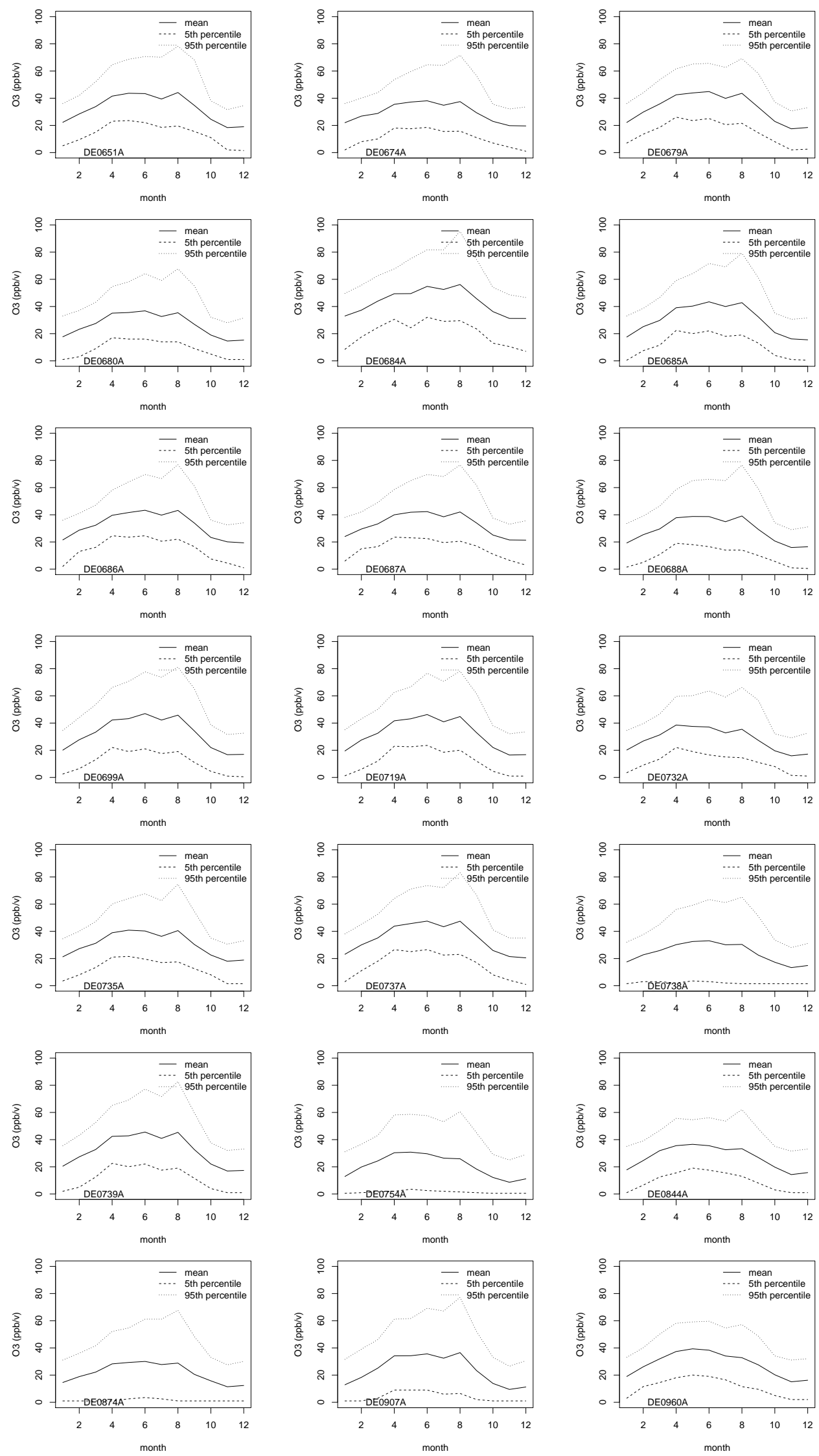

Figure A2: continued. Seasonal cycles of $\mathrm{O}_{3}$ (ppbv) for the period 1996-2005 inclusive 

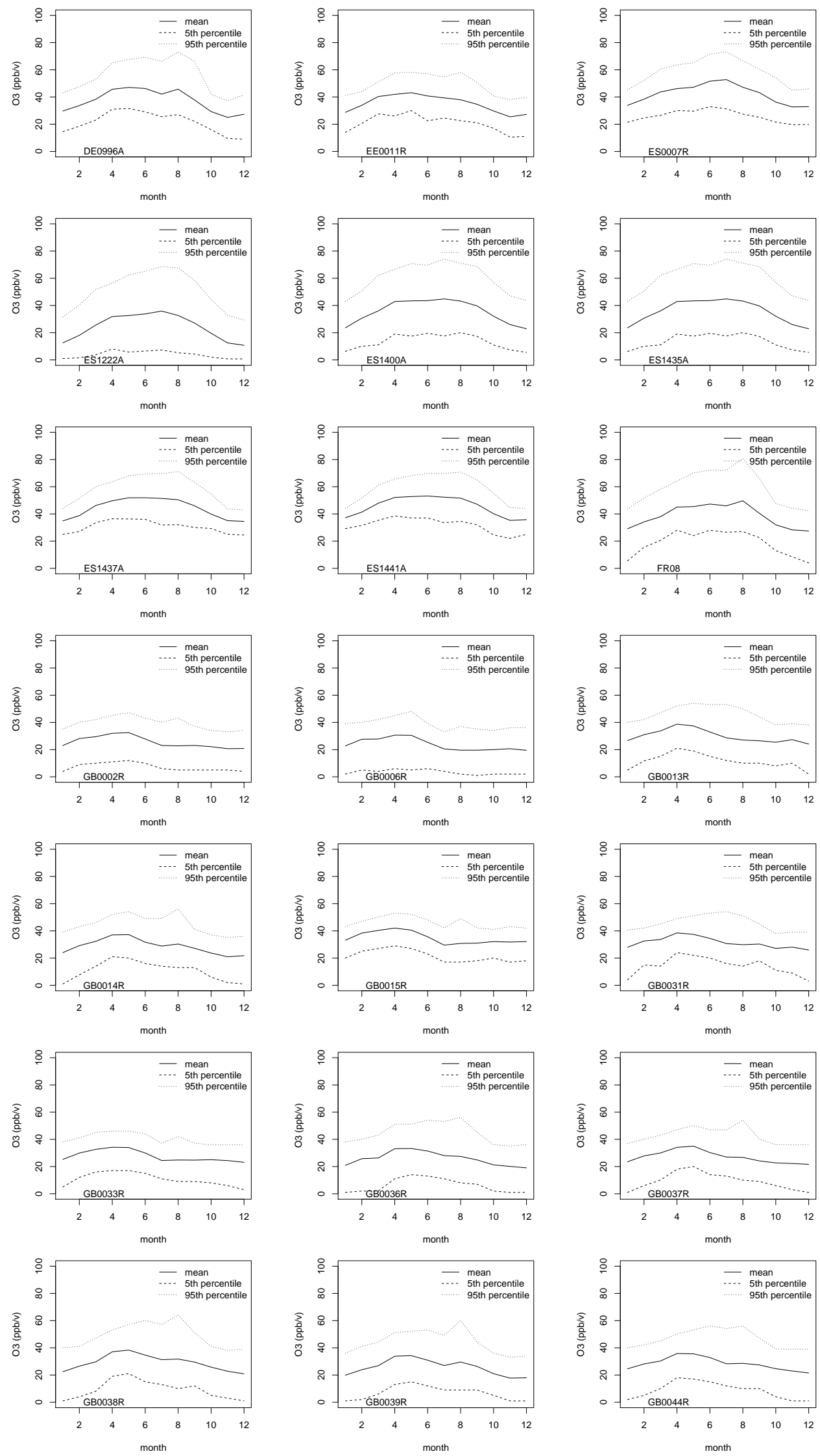

Figure A2: continued. Seasonal cycles of $\mathrm{O}_{3}$ (ppbv) for the period 1996-2005 inclusive 

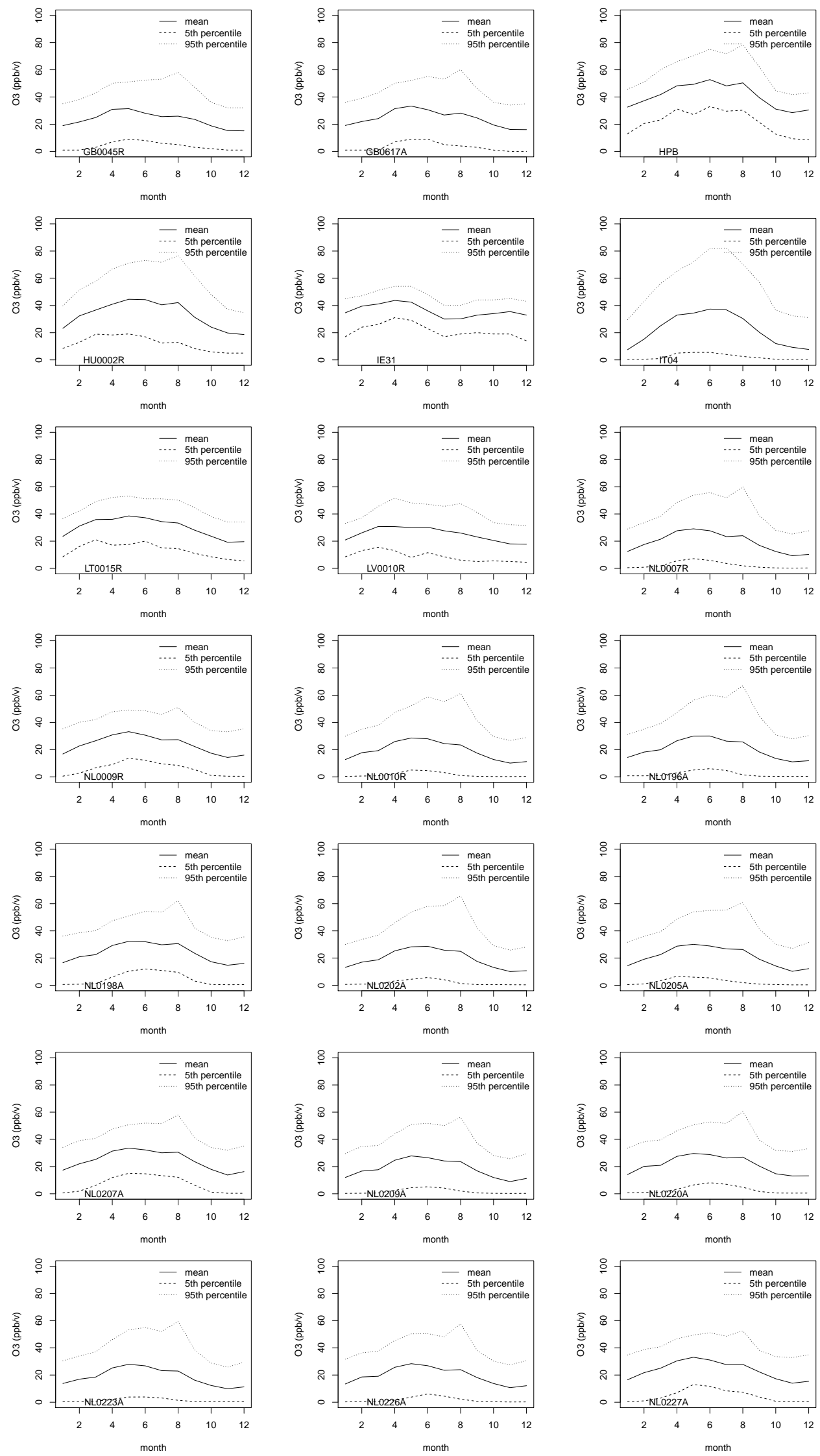

Figure A2: continued. Seasonal cycles of $\mathrm{O}_{3}$ (ppbv) for the period 1996-2005 inclusive 

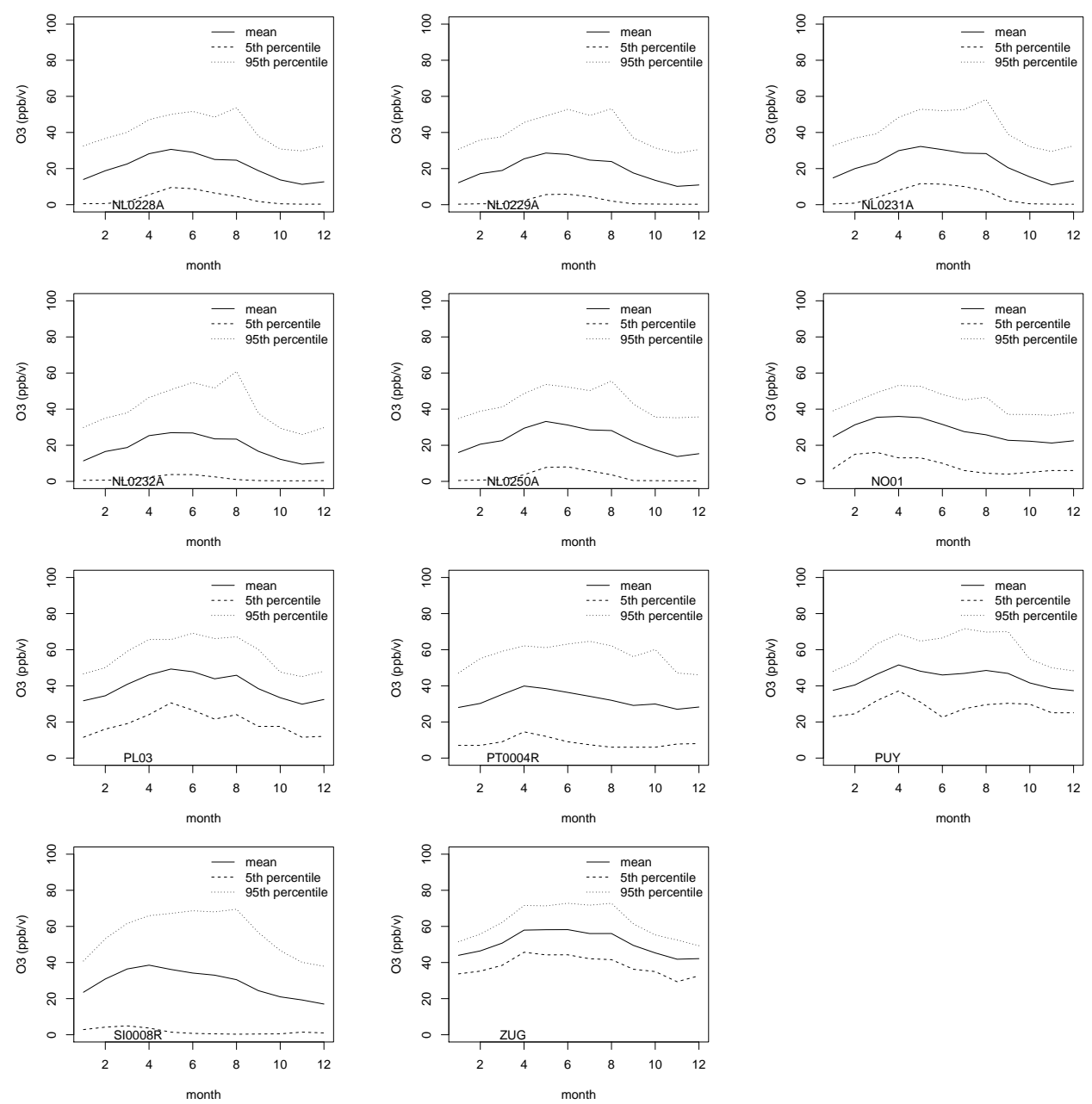

Figure A2: continued. Seasonal cycles of $\mathrm{O}_{3}$ (ppbv) for the period 1996-2005 inclusive 
Table A1: Characterisation and categorisation of monthly mean $\mathrm{O}_{3}$ time series by loess smoothing. NT-no trend, P-positive trend, $\mathrm{N}$-negative trend, I + D increasing then decreasing levels, D + I - decreasing then increasing levels, C - complex behaviour

\begin{tabular}{l|l|l|l|l|l} 
NT & P & N & I + D & D + I & C \\
\hline AT0002R BE0302A DE0674A NL0009R & AT0034R GB0045R & EE0011R & AT0058A & GB0039R & AT0134A \\
AT0004R BE0304A DE0679A NL0010R & AT0052A LT0015R & ES1222A & AT0064A & & AT0180A \\
AT0005R BE0311A DE0685A NL0196A & AT0086A NL0229A & ES1437A & AT0124A & & CH0019A \\
AT0044A BE0345A DE0686A NL0198A & AT0089A & ES1441A & AT0167A & & CZ0055A \\
AT0054A CH0001R DE0687A NL0202A & AT0095A & HU0002R & BE0032R & & DE0684A \\
AT0069A CH0002R DE0688A NL0205A & AT0096A & NO01 & GB0036R & & ES0007R \\
AT0073A CH0003R DE0732A NL0207A & AT0102A & SI0008R & GB0044R & & ES1400A \\
AT0079A CH0004R DE0735A NL0209A & AT0105A & ZUG & PL03 & & GB0002R \\
AT0080A CH0005R DE0737A NL0220A & AT0108A & & & GB0006R \\
AT0094A CH0024A DE0738A NL0223A & AT0111A & & & GB0037R \\
AT0101A CH0033A DE0739A NL0226A & AT0121A & & & LV0010R \\
AT0103A CMN DE0754A NL0227A & AT0154A & & & PT0004R \\
AT0115A CZ0001R DE0844A NL0228A & AT0166A & & & PUY \\
AT0122A CZ0003R DE0874A NL0231A & AT0175A & & & \\
AT0128A CZ0017A DE0907A NL0232A & BE0238A & & & \\
AT0141A CZ0030A DE0960A NL0250A & BE0294A & & & \\
AT0143A CZ0041A DE0996A & CZ0045A & & & \\
AT0146A CZ0049A ES14356 & CZ0051A & & & \\
AT0149A CZ0057A FR08 & CZ0062A & & & \\
AT0153A DE0003R GB0014R & DE0035R & DE0556A & & & \\
AT0162A DE0007R GB0033R & DE0649A & DE0680A & & & \\
AT0164A DE0008R GB0038R & DE0699A & DE0719A & & \\
AT0176A DE0009R GB0617A & GB0013R & & \\
BE0033R DE0422A HPB & GB0031R & & \\
BE0035R DE0510A IE31 & & & \\
BE0211A DE0514A IT04 & & & \\
BE0298A DE0651A NL0007R & & & \\
\hline
\end{tabular}


Table A2: Quantification of trends (ppbv/yr and \%/yr) in $\mathrm{O}_{3}$ monthly mean 19962005. $95 \%$ confidence interval given in brackets. *** significant at $99.9 \%$ level, ** significant at $99.0 \%$ level, ${ }^{*}$ significant at $95.0 \%$ level, + significant at $90.0 \%$ level. ${ }^{\dagger}$ high altitude sites $(>1000 \mathrm{~m}) .{ }^{\ddagger} 2 \sigma$ error. ${ }^{\diamond}$ significant trends only

\begin{tabular}{|c|c|c|c|c|c|c|}
\hline ID & Trend (ppbv/yr) & Sig. & $\mathrm{p}$ value & Trend (\%/yr) & Sig & $\mathrm{p}$ value \\
\hline AT0002R & $0.16(-0.05-0.38)$ & & 0.125 & $0.73(-0.06-1.60)$ & + & 0.073 \\
\hline${ }^{\dagger} \mathrm{AT} 0004 \mathrm{R}$ & $0.19(-0.03-0.42)$ & + & 0.075 & $0.56(-0.11-1.21)$ & + & 0.074 \\
\hline${ }^{\dagger} \mathrm{AT} 0005 \mathrm{R}$ & $0.28(0.00-0.54)$ & $*$ & 0.044 & $0.78(0.03-1.66)$ & $*$ & 0.040 \\
\hline${ }^{\dagger} \mathrm{AT} 0034 \mathrm{R}$ & $0.38(0.18-0.61)$ & $* * *$ & $<0.001$ & $1.42(0.57-2.47)$ & $* *$ & 0.001 \\
\hline AT0044A & $-0.03(-0.27-0.18)$ & & 0.768 & $0.04(-1.01-1.18)$ & & 0.949 \\
\hline AT0052A & $0.36(0.13-0.62)$ & $* *$ & 0.004 & $1.74(0.67-2.71)$ & $* *$ & 0.002 \\
\hline AT0054A & $0.19(0.00-0.41)$ & $*$ & 0.035 & $0.50(-0.11-1.37)$ & & 0.101 \\
\hline${ }^{\dagger}$ AT0058A & $0.15(-0.03-0.33)$ & + & 0.076 & $0.29(-0.12-0.74)$ & & 0.110 \\
\hline${ }^{\dagger} \mathrm{AT} 0064 \mathrm{~A}$ & $0.32(0.10-0.57)$ & $* *$ & 0.002 & $0.73(0.21-1.28)$ & $* *$ & 0.003 \\
\hline AT0069A & $0.07(-0.16-0.32)$ & & 0.496 & $0.34(-0.38-1.18)$ & & 0.312 \\
\hline AT0073A & $-0.02(-0.26-0.19)$ & & 0.852 & $-0.11(-0.81-0.58)$ & & 0.737 \\
\hline${ }^{\dagger} \mathrm{AT} 0079 \mathrm{~A}$ & $0.16(0.02-0.32)$ & $*$ & 0.026 & $0.34(0.04-0.69)$ & $*$ & 0.033 \\
\hline AT0080A & $0.23(0.06-0.44)$ & $*$ & 0.011 & $2.61(1.16-4.04)$ & $* * *$ & $<0.001$ \\
\hline AT0086A & $0.89(0.64-1.16)$ & $* * *$ & $<0.001$ & $4.83(3.56-6.21)$ & $* * *$ & $<0.001$ \\
\hline${ }^{\dagger}$ AT0089A & $0.17(0.01-0.36)$ & $*$ & 0.042 & $0.34(-0.06-0.84)$ & + & 0.087 \\
\hline AT0094A & $0.28(-0.01-0.71)$ & + & 0.072 & $0.74(-0.21-2.01)$ & + & 0.100 \\
\hline AT0095A & $0.70(0.51-0.92)$ & $* * *$ & $<0.001$ & $3.51(2.58-4.55)$ & $* * *$ & $<0.001$ \\
\hline AT0096A & $0.53(0.23-0.84)$ & $* * *$ & $<0.001$ & $2.04(0.92-3.46)$ & $* * *$ & $<0.001$ \\
\hline AT0101A & $0.37(0.17-0.58)$ & $* * *$ & $<0.001$ & $2.04(1.23-2.87)$ & $* * *$ & $<0.001$ \\
\hline AT0102A & $0.65(0.44-0.85)$ & $* * *$ & $<0.001$ & $2.90(2.05-3.80)$ & $* * *$ & $<0.001$ \\
\hline AT0103A & $0.09(-0.11-0.28)$ & & 0.404 & $0.39(-0.38-1.14)$ & & 0.316 \\
\hline AT0105A & $0.69(0.44-0.92)$ & $* * *$ & $<0.001$ & $2.68(1.81-3.55)$ & $* * *$ & $<0.001$ \\
\hline${ }^{\dagger} \mathrm{AT} 0108 \mathrm{~A}$ & $0.37(0.10-0.64)$ & $* *$ & 0.004 & $0.96(0.32-1.73)$ & $* *$ & 0.002 \\
\hline AT0111A & $0.41(0.19-0.64)$ & $* *$ & 0.001 & $1.54(0.51-2.69)$ & $* *$ & 0.003 \\
\hline AT0115A & $0.10(-0.11-0.33)$ & & 0.297 & $0.26(-0.36-0.93)$ & & 0.414 \\
\hline AT0121A & $0.38(0.18-0.61)$ & $* * *$ & $<0.001$ & $1.42(0.57-2.47)$ & $* *$ & 0.001 \\
\hline AT0122A & $0.31(0.06-0.54)$ & $* *$ & 0.006 & $2.62(0.69-4.39)$ & $* *$ & 0.002 \\
\hline${ }^{\dagger} \mathrm{AT} 0124 \mathrm{~A}$ & $0.04(-0.18-0.27)$ & & 0.670 & $0.08(-0.42-0.60)$ & & 0.754 \\
\hline AT0128A & $0.13(-0.09-0.39)$ & & 0.204 & $0.50(-0.66-1.65)$ & & 0.320 \\
\hline AT0134A & $0.82(0.50-1.09)$ & $* * *$ & $<0.001$ & $2.65(1.72-3.60)$ & $* * *$ & $<0.001$ \\
\hline AT0141A & $0.32(0.06-0.55)$ & $* *$ & 0.007 & $2.03(0.65-3.58)$ & $* *$ & 0.002 \\
\hline AT0143A & $0.28(0.12-0.47)$ & $* * *$ & $<0.001$ & $1.18(-0.25-2.81)$ & + & 0.095 \\
\hline${ }^{\dagger}$ AT0146A & $0.02(-0.16-0.25)$ & & 0.810 & $0.06(-0.60-0.74)$ & & 0.828 \\
\hline AT0149A & $0.43(0.26-0.62)$ & $* * *$ & $<0.001$ & $1.75(0.95-2.57)$ & $* * *$ & $<0.001$ \\
\hline AT0153A & $0.18(-0.09-0.51)$ & & 0.181 & $0.65(-0.26-1.68)$ & & 0.126 \\
\hline AT0154A & $1.05(0.73-1.36)$ & $* * *$ & $<0.001$ & $2.88(2.04-3.66)$ & $* * *$ & $<0.001$ \\
\hline AT0162A & $0.16(-0.03-0.37)$ & & 0.123 & $1.54(-0.29-2.96)$ & + & 0.070 \\
\hline AT0164A & $0.39(0.16-0.62)$ & $* * *$ & $<0.001$ & $3.35(2.04-4.79)$ & $* * *$ & $<0.001$ \\
\hline AT0166A & $0.42(0.25-0.63)$ & $* * *$ & $<0.001$ & $2.55(1.29-3.90)$ & $* * *$ & $<0.001$ \\
\hline AT0167A & $0.98(0.62-1.38)$ & $* * *$ & $<0.001$ & $2.25(1.28-3.41)$ & $* * *$ & $<0.001$ \\
\hline AT0175A & $0.46(0.17-0.75)$ & $* * *$ & $<0.001$ & $1.49(0.64-2.46)$ & $* * *$ & $<0.001$ \\
\hline AT0176A & $0.31(0.11-0.53)$ & $* *$ & 0.002 & $0.88(0.36-1.46)$ & $* *$ & 0.001 \\
\hline
\end{tabular}


Table A2: continued. Quantification of trends (ppbv/yr and \%/yr) in $\mathrm{O}_{3}$ monthly mean 1996-2005. 95\% confidence interval given in brackets. *** significant at $99.9 \%$ level, ${ }^{* *}$ significant at $99.0 \%$ level, ${ }^{*}$ significant at $95.0 \%$ level, + significant at $90.0 \%$ level. ${ }^{\dagger}$ high altitude sites $(>1000 \mathrm{~m}) .{ }^{\ddagger} 2 \sigma$ error. ${ }^{\diamond}$ significant trends only

\begin{tabular}{|c|c|c|c|c|c|c|}
\hline ID & Trend (ppbv/yr) & Sig. & $\mathrm{p}$ value & Trend (\%/yr) & Sig & $\mathrm{p}$ value \\
\hline${ }^{\dagger} \mathrm{AT} 0180 \mathrm{~A}$ & $-0.42(-0.69--0.13)$ & $* *$ & 0.001 & $-0.88(-1.42--0.19)$ & $* *$ & 0.006 \\
\hline BE0032R & $0.29(0.05-0.54)$ & $*$ & 0.017 & $1.46(0.26-2.74)$ & $*$ & 0.017 \\
\hline BE0033R & $-0.35(-0.50--0.18)$ & $* * *$ & $<0.001$ & $-3.18(-4.63--1.86)$ & $* * *$ & $<0.001$ \\
\hline BE0035R & $0.26(0.05-0.49)$ & $*$ & 0.013 & $1.55(0.21-2.80)$ & $*$ & 0.023 \\
\hline BE0211A & $0.07(-0.14-0.27)$ & & 0.544 & $-0.71(-2.31-0.71)$ & & 0.357 \\
\hline BE0238A & $0.37(0.22-0.56)$ & $* * *$ & $<0.001$ & $1.95(1.27-2.83)$ & $* * *$ & $<0.001$ \\
\hline BE0294A & $0.04(-0.18-0.30)$ & & 0.771 & $0.47(-0.88-1.72)$ & & 0.532 \\
\hline BE0298A & $0.23(0.00-0.45)$ & $*$ & 0.039 & $0.40(-1.47-1.91)$ & & 0.706 \\
\hline BE0302A & $0.28(0.11-0.47)$ & $* *$ & 0.004 & $1.46(0.62-2.37)$ & $* *$ & 0.001 \\
\hline BE0304A & $0.35(0.10-0.60)$ & $* *$ & 0.005 & $1.60(0.51-2.72)$ & $* *$ & 0.004 \\
\hline BE0311A & $0.01(-0.16-0.20)$ & & 0.898 & $0.46(-0.25-1.08)$ & & 0.249 \\
\hline BE0345A & $-0.31(-0.61--0.05)$ & $*$ & 0.016 & $-3.23(-5.11--1.41)$ & $* * *$ & $<0.001$ \\
\hline${ }^{\dagger} \mathrm{CH} 0001 \mathrm{R}$ & $0.11(-0.02-0.23)$ & + & 0.091 & $0.20(-0.06-0.45)$ & & 0.124 \\
\hline CH0002R & $0.33(0.16-0.55)$ & $* * *$ & $<0.001$ & $2.27(1.29-3.54)$ & $* * *$ & $<0.001$ \\
\hline CH0003R & $0.16(-0.03-0.38)$ & + & 0.099 & $0.94(-0.08-2.24)$ & + & 0.075 \\
\hline${ }^{\dagger} \mathrm{CH} 0004 \mathrm{R}$ & $-0.01(-0.19-0.17)$ & & 0.949 & $0.04(-0.43-0.48)$ & & 0.852 \\
\hline${ }^{\dagger} \mathrm{CH} 0005 \mathrm{R}$ & $0.24(0.04-0.46)$ & $*$ & 0.026 & $0.90(0.33-1.59)$ & $* *$ & 0.007 \\
\hline CH0019A & $0.30(-0.30-0.78)$ & & 0.165 & $1.05(-0.88-2.46)$ & & 0.109 \\
\hline CH0024A & $0.19(0.00-0.41)$ & $*$ & 0.041 & $2.15(0.84-3.96)$ & $* *$ & 0.002 \\
\hline CH0033A & $0.03(-0.12-0.19)$ & & 0.715 & $2.54(1.21-4.30)$ & $* *$ & 0.001 \\
\hline${ }^{\dagger} \mathrm{CMN}$ & $0.08(-0.15-0.28)$ & & 0.460 & $0.10(-0.31-0.47)$ & & 0.574 \\
\hline CZ0001R & $-0.25(-0.48--0.02)$ & $*$ & 0.031 & $-0.73(-1.39-0.04)$ & $*$ & 0.036 \\
\hline CZ0003R & $0.09(-0.10-0.30)$ & & 0.284 & $0.43(-0.27-1.20)$ & & 0.198 \\
\hline CZ0017A & $-0.10(-0.28-0.09)$ & & 0.271 & $-0.17(-0.73-0.50)$ & & 0.647 \\
\hline CZ0030A & $0.13(-0.13-0.38)$ & & 0.295 & $0.42(-0.63-1.39)$ & & 0.348 \\
\hline CZ0041A & $0.40(0.01-0.78)$ & $*$ & 0.034 & $1.38(0.13-2.63)$ & $*$ & 0.030 \\
\hline CZ0045A & $0.44(0.22-0.68)$ & $* * *$ & $<0.001$ & $1.60(0.82-2.46)$ & $* * *$ & $<0.001$ \\
\hline${ }^{\dagger} \mathrm{CZ0049A}$ & $-0.06(-0.27-0.13)$ & & 0.517 & $-0.12(-0.67-0.42)$ & & 0.650 \\
\hline CZ0051A & $0.37(0.17-0.60)$ & $* * *$ & $<0.001$ & $1.37(0.68-2.24)$ & $* * *$ & $<0.001$ \\
\hline${ }^{\dagger} \mathrm{CZ} 0055 \mathrm{~A}$ & $-0.58(-1.07--0.12)$ & $* *$ & 0.005 & $-1.66(-3.12--0.32)$ & $* *$ & 0.004 \\
\hline CZ0057A & $0.04(-0.22-0.30)$ & & 0.734 & $0.09(-0.76-0.86)$ & & 0.789 \\
\hline CZ0062A & $0.53(0.27-0.81)$ & $* * *$ & $<0.001$ & $1.72(0.88-2.66)$ & $* * *$ & $<0.001$ \\
\hline${ }^{\dagger} \mathrm{DE} 0003 \mathrm{R}$ & $0.25(0.01-0.44)$ & $*$ & 0.021 & $0.59(0.02-1.11)$ & $*$ & 0.017 \\
\hline DE0007R & $0.15(-0.05-0.37)$ & & 0.202 & $0.52(-0.52-1.57)$ & & 0.384 \\
\hline DE0008R & $0.23(-0.02-0.47)$ & $*$ & 0.047 & $0.88(-0.02-1.68)$ & $*$ & 0.030 \\
\hline DE0009R & $0.10(-0.09-0.29)$ & & 0.314 & $0.60(-0.26-1.34)$ & & 0.132 \\
\hline DE0035R & $0.33(0.08-0.59)$ & $*$ & 0.013 & $1.63(0.57-2.74)$ & $* *$ & 0.002 \\
\hline DE0422A & $0.31(0.14-0.51)$ & $* * *$ & $<0.001$ & $1.66(0.27-3.24)$ & $*$ & 0.013 \\
\hline DE0510A & $0.13(-0.06-0.36)$ & & 0.148 & $0.69(-0.47-2.09)$ & & 0.211 \\
\hline DE0514A & $0.10(-0.07-0.27)$ & & 0.334 & $0.91(-0.23-1.96)$ & & 0.125 \\
\hline DE0556A & $0.51(0.30-0.72)$ & $* * *$ & $<0.001$ & $1.80(1.18-2.65)$ & $* * *$ & $<0.001$ \\
\hline DE0649A & $0.43(0.24-0.63)$ & $* * *$ & $<0.001$ & $2.52(1.58-3.38)$ & *** & $<0.001$ \\
\hline
\end{tabular}


Table A2: continued. Quantification of trends (ppbv/yr and \%/yr) in $\mathrm{O}_{3}$ monthly mean 1996-2005. 95\% confidence interval given in brackets. *** significant at $99.9 \%$ level, ${ }^{* *}$ significant at $99.0 \%$ level, ${ }^{*}$ significant at $95.0 \%$ level, + significant at $90.0 \%$ level. ${ }^{\dagger}$ high altitude sites $(>1000 \mathrm{~m}) .{ }^{\ddagger} 2 \sigma$ error. ${ }^{\diamond}$ significant trends only

\begin{tabular}{|c|c|c|c|c|c|c|}
\hline ID & Trend (ppbv/yr) & Sig. & $\mathrm{p}$ value & Trend (\%/yr) & Sig & $\mathrm{p}$ value \\
\hline DE0651A & $0.29(0.09-0.53)$ & $*$ & 0.011 & $1.30(0.62-2.10)$ & $* *$ & 0.001 \\
\hline DE0674A & $0.09(-0.06-0.33)$ & & 0.244 & $0.81(0.03-1.65)$ & + & 0.051 \\
\hline DE0679A & $0.11(-0.08-0.33)$ & & 0.247 & $0.70(-0.17-1.52)$ & + & 0.075 \\
\hline DE0680A & $0.38(0.20-0.61)$ & $* * *$ & $<0.001$ & $1.96(1.10-3.05)$ & $* * *$ & $<0.001$ \\
\hline DE0684A & $-0.46(-0.84--0.06)$ & $*$ & 0.012 & $-1.03(-2.03--0.08)$ & $*$ & 0.018 \\
\hline DE0685A & $0.30(0.14-0.54)$ & $* * *$ & $<0.001$ & $1.60(0.88-2.50)$ & $* * *$ & $<0.001$ \\
\hline DE0686A & $0.04(-0.12-0.26)$ & & 0.552 & $0.27(-0.46-0.99)$ & & 0.488 \\
\hline DE0687A & $0.16(-0.02-0.39)$ & + & 0.070 & $0.74(0.09-1.53)$ & $*$ & 0.014 \\
\hline DE0688A & $0.16(-0.05-0.39)$ & & 0.140 & $1.12(0.26-2.17)$ & $*$ & 0.011 \\
\hline DE0699A & $0.44(0.19-0.71)$ & $* * *$ & $<0.001$ & $1.88(0.94-2.89)$ & $* * *$ & $<0.001$ \\
\hline DE0719A & $0.54(0.31-0.80)$ & $* * *$ & $<0.001$ & $2.44(1.58-3.42)$ & $* * *$ & $<0.001$ \\
\hline DE0732A & $0.03(-0.16-0.23)$ & & 0.806 & $0.49(-0.36-1.43)$ & & 0.261 \\
\hline DE0735A & $0.31(0.13-0.51)$ & $* * *$ & $<0.001$ & $1.45(0.70-2.11)$ & $* * *$ & $<0.001$ \\
\hline DE0737A & $-0.15(-0.32-0.05)$ & & 0.107 & $0.03(-0.66-0.75)$ & & 0.949 \\
\hline DE0738A & $0.25(0.08-0.42)$ & $* *$ & 0.003 & $1.79(0.76-2.73)$ & $* * *$ & $<0.001$ \\
\hline DE0739A & $0.22(0.01-0.43)$ & $*$ & 0.038 & $1.28(0.48-2.13)$ & $* *$ & 0.002 \\
\hline DE0754A & $-0.06(-0.25-0.16)$ & & 0.627 & $0.59(-0.94-2.10)$ & & 0.441 \\
\hline DE0844A & $0.11(-0.06-0.25)$ & & 0.226 & $0.70(-0.03-1.34)$ & + & 0.067 \\
\hline DE0874A & $0.23(0.05-0.41)$ & $* *$ & 0.009 & $2.16(1.04-3.28)$ & $* * *$ & $<0.001$ \\
\hline DE0907A & $0.44(0.16-0.72)$ & $* *$ & 0.003 & $2.49(1.07-3.89)$ & $* *$ & 0.002 \\
\hline DE0960A & $0.07(-0.14-0.29)$ & & 0.525 & $0.15(-0.77-1.09)$ & & 0.764 \\
\hline DE0996A & $0.20(0.01-0.40)$ & $*$ & 0.042 & $0.65(0.08-1.26)$ & $*$ & 0.022 \\
\hline EE0011R & $-0.38(-0.57--0.17)$ & $* * *$ & $<0.001$ & $-1.15(-1.79--0.52)$ & $* * *$ & $<0.001$ \\
\hline${ }^{\dagger} \mathrm{ES} 0007 \mathrm{R}$ & $-0.54(-1.09--0.03)$ & $*$ & 0.017 & $-1.48(-2.96--0.19)$ & $*$ & 0.010 \\
\hline ES1222A & $-0.38(-0.54--0.25)$ & $* * *$ & $<0.001$ & $-2.55(-3.85--1.58)$ & $* * *$ & $<0.001$ \\
\hline ES1400A & $-1.02(-1.36--0.63)$ & $* * *$ & $<0.001$ & $-3.27(-4.49--2.17)$ & $* * *$ & $<0.001$ \\
\hline${ }^{\dagger} \mathrm{ES} 1435 \mathrm{~A}$ & $0.10(-0.07-0.33)$ & & 0.263 & $0.22(-0.28-0.74)$ & & 0.378 \\
\hline${ }^{\dagger} \mathrm{ES} 1437 \mathrm{~A}$ & $-0.54(-0.75--0.35)$ & $* * *$ & $<0.001$ & $-1.39(-1.88--0.94)$ & $* * *$ & $<0.001$ \\
\hline${ }^{\dagger} \mathrm{ES} 1441 \mathrm{~A}$ & $-0.19(-0.37--0.03)$ & $*$ & 0.041 & $-0.45(-0.89--0.06)$ & $*$ & 0.036 \\
\hline FR08 & $0.29(-0.01-0.58)$ & $*$ & 0.036 & $0.95(0.14-1.99)$ & $*$ & 0.024 \\
\hline GB0002R & $-0.12(-0.28-0.04)$ & & 0.198 & $-0.24(-1.03-0.51)$ & & 0.540 \\
\hline GB0006R & $0.26(0.02-0.50)$ & $*$ & 0.015 & $1.72(0.43-2.87)$ & $* *$ & 0.002 \\
\hline GB0013R & $0.18(0.01-0.36)$ & + & 0.071 & $0.70(-0.06-1.43)$ & + & 0.075 \\
\hline GB0014R & $0.21(0.01-0.40)$ & $*$ & 0.039 & $1.15(0.29-1.94)$ & $* *$ & 0.006 \\
\hline GB0015R & $0.23(0.04-0.44)$ & $*$ & 0.019 & $0.60(0.02-1.27)$ & $*$ & 0.045 \\
\hline GB0031R & $0.43(0.21-0.67)$ & $* * *$ & $<0.001$ & $1.59(0.81-2.46)$ & $* * *$ & $<0.001$ \\
\hline GB0033R & $0.07(-0.09-0.24)$ & & 0.404 & $0.48(-0.19-1.24)$ & & 0.132 \\
\hline GB0036R & $0.21(-0.01-0.49)$ & + & 0.060 & $1.52(0.39-2.84)$ & $* *$ & 0.009 \\
\hline GB0037R & $0.14(-0.14-0.40)$ & & 0.314 & $0.90(-0.20-1.89)$ & & 0.101 \\
\hline GB0038R & $0.20(0.00-0.42)$ & + & 0.078 & $0.95(0.11-1.89)$ & $*$ & 0.044 \\
\hline GB0039R & $0.30(0.07-0.49)$ & $* *$ & 0.006 & $1.41(0.45-2.40)$ & $* *$ & 0.005 \\
\hline GB0044R & $-0.07(-0.23-0.14)$ & & 0.453 & $-0.07(-0.82-0.88)$ & & 0.881 \\
\hline
\end{tabular}


Table A2: continued. Quantification of trends (ppbv/yr and \%/yr) in $\mathrm{O}_{3}$ monthly mean 1996-2005. 95\% confidence interval given in brackets. *** significant at $99.9 \%$ level, $* *$ significant at $99.0 \%$ level, $*$ significant at $95.0 \%$ level, + significant at $90.0 \%$ level. ${ }^{\dagger}$ high altitude sites $(>1000 \mathrm{~m}) .{ }^{\ddagger} 2 \sigma$ error. ${ }^{\diamond}$ significant trends only

\begin{tabular}{|c|c|c|c|c|c|c|}
\hline ID & Trend (ppbv/yr) & Sig. & $\mathrm{p}$ value & Trend (\%/yr) & Sig & $\mathrm{p}$ value \\
\hline GB0045R & $0.65(0.46-0.86)$ & $* * *$ & $<0.001$ & $3.67(2.54-5.06)$ & $* * *$ & $<0.001$ \\
\hline GB0617A & $0.00(-0.18-0.18)$ & & 0.962 & $0.35(-0.69-1.28)$ & & 0.552 \\
\hline HPB & $0.16(-0.04-0.34)$ & + & 0.085 & $0.44(-0.14-1.01)$ & & 0.101 \\
\hline HU0002R & $-1.28(-1.64--0.87)$ & $* * *$ & $<0.001$ & $-4.11(-5.46--2.89)$ & $* * *$ & $<0.001$ \\
\hline IE31 & $0.09(-0.08-0.33)$ & & 0.251 & $0.32(-0.22-1.08)$ & & 0.247 \\
\hline IT04 & $0.23(-0.04-0.51)$ & + & 0.080 & $6.05(3.94-8.74)$ & $* * *$ & $<0.001$ \\
\hline LT0015R & $0.31(0.13-0.50)$ & $* *$ & 0.001 & $1.14(0.41-1.76)$ & $* *$ & 0.004 \\
\hline LV0010R & $-0.33(-0.53--0.16)$ & $* *$ & 0.003 & $-1.92(-2.84--1.04)$ & $* * *$ & $<0.001$ \\
\hline NL0007R & $0.12(-0.05-0.29)$ & & 0.209 & $0.70(-0.45-1.86)$ & & 0.244 \\
\hline NL0009R & $0.32(0.09-0.52)$ & $* *$ & 0.002 & $1.52(0.50-2.71)$ & $* *$ & 0.004 \\
\hline NL0010R & $0.20(0.03-0.37)$ & $*$ & 0.024 & $0.08(-1.35-1.43)$ & & 0.895 \\
\hline NL0196A & $0.06(-0.13-0.26)$ & & 0.534 & $-0.24(-1.52-1.28)$ & & 0.766 \\
\hline NL0198A & $0.13(-0.06-0.33)$ & & 0.172 & $0.90(-0.36-2.08)$ & & 0.120 \\
\hline NL0202A & $0.25(0.04-0.47)$ & $*$ & 0.021 & $1.90(0.23-3.46)$ & * & 0.019 \\
\hline NL0205A & $0.22(0.01-0.43)$ & $*$ & 0.033 & $0.76(-0.50-1.75)$ & & 0.217 \\
\hline NL0207A & $0.20(-0.04-0.41)$ & + & 0.074 & $1.18(0.24-2.21)$ & $*$ & 0.013 \\
\hline NL0209A & $0.21(0.01-0.44)$ & $*$ & 0.043 & $2.02(0.43-4.03)$ & $*$ & 0.015 \\
\hline NL0220A & $0.02(-0.14-0.24)$ & & 0.828 & $0.36(-0.76-1.59)$ & & 0.543 \\
\hline NL0223A & $0.08(-0.08-0.26)$ & & 0.400 & $1.33(0.29-2.70)$ & $*$ & 0.043 \\
\hline NL0226A & $0.08(-0.06-0.24)$ & & 0.250 & $0.04(-1.44-1.31)$ & & 0.924 \\
\hline NL0227A & $0.05(-0.13-0.27)$ & & 0.640 & $0.47(-0.75-1.75)$ & & 0.451 \\
\hline NL0228A & $0.22(0.05-0.42)$ & $*$ & 0.013 & $1.05(0.02-2.18)$ & + & 0.057 \\
\hline NL0229A & $0.45(0.27-0.65)$ & $* * *$ & $<0.001$ & $3.56(2.21-4.93)$ & $* * *$ & $<0.001$ \\
\hline NL0231A & $0.09(-0.18-0.35)$ & & 0.503 & $0.97(-0.42-2.22)$ & & 0.120 \\
\hline NL0232A & $0.05(-0.12-0.26)$ & & 0.608 & $0.01(-1.40-1.57)$ & & 0.993 \\
\hline NL0250A & $0.15(-0.07-0.40)$ & & 0.183 & $0.72(-0.69-2.24)$ & & 0.396 \\
\hline NO01 & $-0.16(-0.31--0.03)$ & $*$ & 0.022 & $-0.87(-1.50--0.32)$ & $* *$ & 0.001 \\
\hline${ }^{\dagger} \mathrm{PL03}$ & $-0.43(-0.78--0.12)$ & $* *$ & 0.003 & $-1.38(-2.44--0.42)$ & $* *$ & 0.003 \\
\hline PT0004R & $0.32(-0.39-0.96)$ & & 0.263 & $0.30(-2.04-2.86)$ & & 0.750 \\
\hline${ }^{\dagger} \mathrm{PUY}$ & $0.03(-0.21-0.28)$ & & 0.821 & $0.16(-0.40-0.75)$ & & 0.577 \\
\hline SI0008R & $-0.24(-0.48-0.03)$ & $*$ & 0.045 & $-2.22(-3.55--0.64)$ & $* *$ & 0.001 \\
\hline${ }^{\dagger} \mathrm{ZUG}$ & $-0.29(-0.41--0.17)$ & $* * *$ & $<0.001$ & $-0.57(-0.83--0.28)$ & $* * *$ & $<0.001$ \\
\hline $\begin{array}{c}\text { European } \\
\text { average }^{\ddagger}\end{array}$ & $0.16 \pm 0.02 \mathrm{ppbv} / \mathrm{yr}$ & & & $0.83 \pm 0.08 \% / \mathrm{yr}$ & & \\
\hline Range $^{\diamond}$ & -1.28 to $1.05 \mathrm{ppbv} / \mathrm{yr}$ & & & -4.12 to $6.05 \% / y r$ & & \\
\hline
\end{tabular}


Table A3: Quantification of trends (ppbv/yr and \%/yr) in $\mathrm{O}_{3}$ monthly $5^{\text {th }}$ percentiles 1996-2005. 95\% confidence interval given in brackets. *** significant at $99.9 \%$ level, ** significant at $99.0 \%$ level, ${ }^{*}$ significant at $95.0 \%$ level, + significant at $90.0 \%$ level. $\dagger$ high altitude sites $(>1000 \mathrm{~m})$. ${ }^{\ddagger} 2 \sigma$ error. ${ }^{\diamond}$ significant trends only

\begin{tabular}{|c|c|c|c|c|c|c|}
\hline ID & Trend (ppbv/yr) & Sig. & $\mathrm{p}$ value & Trend (\%/yr) & Sig & $\mathrm{p}$ value \\
\hline AT0002R & $0.09(-0.05-0.25)$ & & 0.212 & $1.75(0.16-3.67)$ & $*$ & 0.017 \\
\hline${ }^{\dagger} \mathrm{AT} 0004 \mathrm{R}$ & $0.33(0.03-0.65)$ & * & 0.038 & $1.44(-0.26-3.25)$ & + & 0.090 \\
\hline${ }^{\dagger} \mathrm{AT} 0005 \mathrm{R}$ & $0.31(0.06-0.60)$ & $*$ & 0.019 & $1.58(0.38-3.09)$ & $*$ & 0.019 \\
\hline${ }^{\dagger} \mathrm{AT} 0034 \mathrm{R}$ & $0.47(0.22-0.72)$ & $* * *$ & $<0.001$ & $3.88(1.49-6.16)$ & $* * *$ & $<0.001$ \\
\hline AT0044A & $0.02(-0.11-0.13)$ & & 0.779 & $1.10(-1.25-3.50)$ & & 0.381 \\
\hline AT0052A & $0.43(0.13-0.69)$ & $* * *$ & $<0.001$ & $4.87(2.43-7.40)$ & $* * *$ & $<0.001$ \\
\hline AT0054A & $0.20(-0.05-0.46)$ & + & 0.088 & $0.93(-0.49-2.29)$ & & 0.162 \\
\hline${ }^{\dagger} \mathrm{AT} 0058 \mathrm{~A}$ & $0.03(-0.22-0.27)$ & & 0.782 & $0.06(-0.64-0.86)$ & & 0.852 \\
\hline${ }^{\dagger} \mathrm{AT} 0064 \mathrm{~A}$ & $0.33(0.02-0.63)$ & $*$ & 0.029 & $0.74(-0.11-1.74)$ & + & 0.082 \\
\hline AT0069A & $0.17(-0.03-0.38)$ & + & 0.095 & $1.01(-0.44-2.89)$ & & 0.206 \\
\hline AT0073A & $-0.06(-0.32-0.25)$ & & 0.666 & $-0.48(-1.88-0.96)$ & & 0.493 \\
\hline${ }^{\dagger} \mathrm{AT} 0079 \mathrm{~A}$ & $0.06(-0.12-0.25)$ & & 0.490 & $0.15(-0.33-0.61)$ & & 0.528 \\
\hline AT0080A & $0.03(0.00-0.07)$ & $* * *$ & $<0.001$ & $0.79(0.14-2.79)$ & $* * *$ & $<0.001$ \\
\hline AT0086A & $0.59(0.40-0.83)$ & $* * *$ & $<0.001$ & $7.05(5.01-9.09)$ & $* * *$ & $<0.001$ \\
\hline${ }^{\dagger} \mathrm{AT} 0089 \mathrm{~A}$ & $0.14(-0.14-0.45)$ & & 0.341 & $0.41(-0.44-1.34)$ & & 0.369 \\
\hline AT0094A & $0.34(0.01-0.74)$ & $*$ & 0.044 & $2.40(-0.04-5.35)$ & $*$ & 0.033 \\
\hline AT0095A & $0.45(0.29-0.64)$ & $* * *$ & $<0.001$ & $6.08(4.03-8.12)$ & $* * *$ & $<0.001$ \\
\hline AT0096A & $0.54(0.34-0.73)$ & $* * *$ & $<0.001$ & $4.44(2.63-6.78)$ & $* * *$ & $<0.001$ \\
\hline AT0101A & $0.50(0.30-0.70)$ & $* * *$ & $<0.001$ & $5.49(3.58-7.73)$ & ** & $<0.001$ \\
\hline AT0102A & $0.49(0.33-0.71)$ & $* * *$ & $<0.001$ & $5.01(3.20-7.39)$ & $* * *$ & $<0.001$ \\
\hline AT0103A & $0.18(0.01-0.34)$ & $*$ & 0.023 & $1.82(-0.07-3.75)$ & + & 0.053 \\
\hline AT0105A & $0.73(0.53-0.94)$ & $* * *$ & $<0.001$ & $4.89(3.34-6.80)$ & $* * *$ & $<0.001$ \\
\hline${ }^{\dagger} \mathrm{AT} 0108 \mathrm{~A}$ & $0.50(0.19-0.80)$ & $* * *$ & $<0.001$ & $1.76(0.64-2.90)$ & $* * *$ & $<0.001$ \\
\hline AT0111A & $0.29(0.16-0.44)$ & $* * *$ & $<0.001$ & $2.36(0.62-3.94)$ & $* *$ & 0.007 \\
\hline AT0115A & $0.25(-0.05-0.54)$ & + & 0.057 & $0.86(-0.68-2.36)$ & & 0.211 \\
\hline AT0121A & $0.47(0.22-0.72)$ & & $<0.001$ & $3.88(1.49-6.16)$ & $* * *$ & $<0.001$ \\
\hline AT0122A & $0.16(0.06-0.28)$ & $* * *$ & $<0.001$ & $4.16(0.73-7.36)$ & $*$ & 0.011 \\
\hline${ }^{\dagger} \mathrm{AT} 0124 \mathrm{~A}$ & $-0.04(-0.30-0.23)$ & & 0.775 & $-0.04(-0.75-0.69)$ & & 0.931 \\
\hline AT0128A & $0.02(-0.11-0.14)$ & & 0.765 & $1.81(-0.49-4.44)$ & & 0.121 \\
\hline AT0134A & $0.67(0.40-0.98)$ & $* * *$ & $<0.001$ & $3.72(2.08-5.47)$ & $* * *$ & $<0.001$ \\
\hline AT0141A & $0.14(0.02-0.26)$ & $*$ & 0.013 & $2.93(-0.63-6.27)$ & + & 0.054 \\
\hline AT0143A & $0.00(-0.06-0.09)$ & & 0.764 & $-0.01(-4.36-4.03)$ & & 0.987 \\
\hline${ }^{\dagger} \mathrm{AT} 0146 \mathrm{~A}$ & $-0.05(-0.32-0.29)$ & & 0.751 & $-0.57(-2.14-1.00)$ & & 0.396 \\
\hline AT0149A & $0.44(0.27-0.60)$ & $* * *$ & $<0.001$ & $3.40(1.86-4.95)$ & $* * *$ & $<0.001$ \\
\hline AT0153A & $0.28(0.00-0.57)$ & $*$ & 0.040 & $1.14(-0.10-2.74)$ & + & 0.065 \\
\hline AT0154A & $0.98(0.69-1.26)$ & $* * *$ & $<0.001$ & $4.31(3.15-5.68)$ & $* * *$ & $<0.001$ \\
\hline AT0162A & $0.13(0.03-0.21)$ & $* *$ & 0.002 & $3.39(-0.58-6.97)$ & + & 0.070 \\
\hline AT0164A & $0.26(0.19-0.37)$ & $* * *$ & $<0.001$ & $9.07(6.59-11.83)$ & $* * *$ & $<0.001$ \\
\hline AT0166A & $0.27(0.12-0.44)$ & $* * *$ & $<0.001$ & $4.94(1.82-8.40)$ & $* *$ & 0.002 \\
\hline AT0167A & $0.98(0.57-1.35)$ & $* * *$ & $<0.001$ & $3.50(1.68-5.22)$ & $* * *$ & $<0.001$ \\
\hline AT0175A & $0.46(0.20-0.77)$ & $* * *$ & $<0.001$ & $2.61(1.22-4.09)$ & $* * *$ & $<0.001$ \\
\hline AT0176A & $0.41(0.18-0.63)$ & $* * *$ & $<0.001$ & $1.76(0.86-3.06)$ & $* * *$ & $<0.001$ \\
\hline
\end{tabular}


Table A3: continued. Quantification of trends (ppbv/yr and $\% / \mathrm{yr}$ ) in $\mathrm{O}_{3}$ monthly $5^{\text {th }}$ percentiles 1996-2005. 95\% confidence interval given in brackets. *** significant at 99.9\% level, ${ }^{* *}$ significant at $99.0 \%$ level, ${ }^{*}$ significant at $95.0 \%$ level, + significant at $90.0 \%$ level. ${ }^{\dagger}$ high altitude sites $(>1000 \mathrm{~m}) . \stackrel{\ddagger}{ } 2 \sigma$ error. ${ }^{\diamond}$ significant trends only

\begin{tabular}{|c|c|c|c|c|c|c|}
\hline ID & Trend (ppbv/yr) & Sig. & $\mathrm{p}$ value & Trend $(\% / y r)$ & Sig & $\mathrm{p}$ value \\
\hline${ }^{\dagger} \mathrm{AT} 0180 \mathrm{~A}$ & $-0.53(-0.79--0.22)$ & $* *$ & 0.002 & $-1.53(-2.31--0.64)$ & $* *$ & 0.004 \\
\hline BE0032R & $0.12(-0.01-0.25)$ & + & 0.085 & $2.50(-0.10-5.14)$ & + & 0.061 \\
\hline BE0033R & $-0.09(-0.19-0.00)$ & $*$ & 0.037 & $-6.32(-10.53--2.54)$ & $* * *$ & $<0.001$ \\
\hline BE0035R & $0.00(-0.05-0.05)$ & & 0.892 & $0.30(-1.99-3.06)$ & & 0.522 \\
\hline BE0211A & $-0.05(-0.10-0.00)$ & $*$ & 0.040 & $-4.51(-7.24--1.87)$ & $* *$ & 0.003 \\
\hline BE0238A & $0.41(0.27-0.57)$ & $* * *$ & $<0.001$ & $5.05(2.98-6.97)$ & $* * *$ & $<0.001$ \\
\hline BE0294A & $-0.01(-0.10-0.08)$ & & 0.854 & $-1.84(-5.20-0.96)$ & & 0.279 \\
\hline BE0298A & $-0.04(-0.08--0.01)$ & $*$ & 0.012 & $-2.90(-6.02--0.57)$ & $*$ & 0.010 \\
\hline BE0302A & $0.12(0.01-0.24)$ & * & 0.027 & $3.18(0.84-5.67)$ & $* *$ & 0.005 \\
\hline BE0304A & $0.19(0.04-0.35)$ & * & 0.011 & $2.43(-0.28-4.83)$ & + & 0.054 \\
\hline BE0311A & $0.06(-0.1$ & & 0.637 & $0.61(-0.68-2.08)$ & & 0.479 \\
\hline BE0345A & $-0.06(-0.15-0.00)$ & & 0.110 & $-5.74(-10.18--1.09)$ & $* *$ & 0.004 \\
\hline${ }^{\dagger} \mathrm{CH} 0001 \mathrm{R}$ & $-0.01(-0.20-0.18)$ & & & $-0.01(-0.52-0.48)$ & & 0.960 \\
\hline CH0002R & $0.37(0.23-0.49)$ & $* * *$ & $<0.001$ & $7.21(5.11-9.56)$ & $* * *$ & $<0.001$ \\
\hline CH0003R & $0.16(0.06-0.26)$ & $* *$ & & $2.98(0.78-5.06)$ & $* *$ & 0.006 \\
\hline${ }^{\dagger} \mathrm{CH} 0004 \mathrm{R}$ & $0.12(-0.08-0.35)$ & & 0.280 & $0.58(-0.27-1.63)$ & & 0.212 \\
\hline${ }^{\dagger} \mathrm{CH} 0005 \mathrm{R}$ & $0.53(0.25-0.87)$ & $* * *$ & & $2.69(1.30-4.61)$ & $* * *$ & $<0.001$ \\
\hline CH0019A & $0.44(0.01-0.92)$ & $*$ & 21 & $2.73(-1.31-5.89)$ & + & 0.068 \\
\hline CH0024A & $0.09(0.04-0.15)$ & $* * *$ & $<0.001$ & $4.88(2.42-8.05)$ & $* * *$ & $<0.001$ \\
\hline CH0033A & $0.07(0.04-0.12)$ & $* * *$ & $<0.001$ & $8.45(5.16-12.38)$ & $* * *$ & $<0.001$ \\
\hline${ }^{\dagger} \mathrm{CMN}$ & $0.02(-0.18-0.22)$ & & 849 & $0.00(-0.55-0.48)$ & & 0.975 \\
\hline CZ0001R & -0.09( & & & $-0.34(-1.55-0.88)$ & & 0.561 \\
\hline CZ0003R & $0.18(-0.01-0.38)$ & + & 0.060 & $1.56(0.08-2.92)$ & * & 0.024 \\
\hline CZ0017A & $-0.04(-0.31-0.24)$ & & 0.7 & $-0.22(-1.58-1.18)$ & & 0.736 \\
\hline CZ0030A & $-0.09(-0.36-0.17)$ & & 0.406 & $-0.03(-2.11-2.37)$ & & 0.967 \\
\hline CZ0041A & $0.18(-0.11-0.49)$ & & 0.193 & $2.06(0.14-3.97)$ & $*$ & 0.033 \\
\hline CZ0045A & $0.43(0.21-0.70)$ & $* * *$ & $<0.001$ & $2.76(1.23-4.52)$ & $* * *$ & $<0.001$ \\
\hline${ }^{\dagger} \mathrm{CZ} 0049 \mathrm{~A}$ & $0.07(-0.16-0.33)$ & & & $0.17(-0.93-1.48)$ & & 0.700 \\
\hline CZ0051A & $0.57(0.3$ & $* * *$ & & $2.74(1.54-4.14)$ & & $<0.001$ \\
\hline${ }^{\dagger} \mathrm{CZ} 0055 \mathrm{~A}$ & $-0.64(-1.06--0.22)$ & $* *$ & 0.001 & $-3.06(-4.97--1.18)$ & $* * *$ & 0.001 \\
\hline CZ0057A & $0.10(-0.16-0.38)$ & & 0.401 & $0.82(-0.72-2.32)$ & & 0.257 \\
\hline CZ0062A & $0.39(0.16-0.62)$ & $* * *$ & $<0.001$ & $2.59(1.06-4.22)$ & $* * *$ & $<0.001$ \\
\hline${ }^{\dagger} \mathrm{DE} 0003 \mathrm{R}$ & $0.15(-0.12-0.37)$ & & 0.264 & $0.30(-0.72-1.26)$ & & 0.565 \\
\hline DE0007R & $-0.06(-0.20-0.08)$ & & 0.348 & $-0.37(-3.35-2.56)$ & & 0.835 \\
\hline DE0008R & $0.24(-0.01-0.48)$ & $*$ & 0.033 & $1.32(-0.02-2.85)$ & $*$ & 0.033 \\
\hline DE0009R & $0.11(-0.0$ & & & $1.10(-0.18-3.06)$ & & 0.101 \\
\hline DE0035R & $0.27(0.03-0.50)$ & * & 0.027 & $2.31(0.42-4.78)$ & $*$ & 0.016 \\
\hline DE0422A & $0.00(0.00-0.00)$ & & 0.445 & $-0.67(-0.72--0.42)$ & $* * *$ & $<0.001$ \\
\hline DE0510A & $0.01(-0.01-0.08)$ & & & $0.10(-0.70-1.61)$ & & 0.796 \\
\hline & $0.00(-0.03-0.02)$ & & 0.391 & $-0.03(-0.59-0.64)$ & & 0.608 \\
\hline DE0556A & $0.38(0.19-0.60)$ & $* * *$ & $<0.001$ & $2.78(1.17-5.19)$ & $* * *$ & $<0.001$ \\
\hline DE0649A & $0.18(0.10-0.31)$ & $* *$ & $<0.001$ & $4.40(2.77-6.02)$ & $* * *$ & $<0.001$ \\
\hline
\end{tabular}


Table A3: continued. Quantification of trends (ppbv/yr and $\% / \mathrm{yr}$ ) in $\mathrm{O}_{3}$ monthly $5^{\text {th }}$ percentiles 1996-2005. 95\% confidence interval given in brackets. *** significant at 99.9\% level, ${ }^{* *}$ significant at $99.0 \%$ level, ${ }^{*}$ significant at $95.0 \%$ level, + significant at $90.0 \%$ level. ${ }^{\dagger}$ high altitude sites $(>1000 \mathrm{~m}) .{ }^{\ddagger} 2 \sigma$ error. ${ }^{\diamond}$ significant trends only

\begin{tabular}{|c|c|c|c|c|c|c|}
\hline ID & Trend (ppbv/yr) & Sig. & $\mathrm{p}$ value & Trend $(\% / y r)$ & Sig & $\mathrm{p}$ value \\
\hline DE0651A & $0.25(0.07-0.44)$ & $* *$ & 0.009 & $2.43(0.74-4.21)$ & $* *$ & 0.004 \\
\hline DE0674A & $0.31(0.09-0.51)$ & $* *$ & 0.002 & $2.70(0.98-4.64)$ & $* *$ & 0.002 \\
\hline DE0679A & $0.18(-0.03-0.45)$ & + & 0.091 & $1.94(0.16-3.90)$ & * & 0.022 \\
\hline DE0680A & $0.41(0.27-0.56)$ & $* * *$ & $<0.001$ & $3.70(2.05-5.54)$ & $* * *$ & $<0.001$ \\
\hline DE0684A & $-0.34(-0.73-0.05)$ & + & 0.053 & $-1.50(-2.94-0.36)$ & + & 0.072 \\
\hline DE0685A & $0.34(0.19-0.53)$ & $* * *$ & $<0.001$ & $4.20(2.59-6.33)$ & $* * *$ & $<0.001$ \\
\hline DE0686A & $0.11(-0.10-0.30)$ & & 0.235 & $0.46(-0.96-2.49)$ & & 0.474 \\
\hline DE0687A & $0.25(0.04-0.48)$ & $*$ & 0.013 & $1.91(0.51-3.82)$ & $* *$ & 0.007 \\
\hline DE0688A & $0.34(0.15-0.52)$ & $* * *$ & $<0.001$ & $4.48(2.41-6.82)$ & $* * *$ & $<0.001$ \\
\hline DE0699A & $0.35(0.15-0.57)$ & $* * *$ & $<0.001$ & $6.02(3.66-8.51)$ & $* * *$ & $<0.001$ \\
\hline DE0719A & $0.45(0.29-0.66)$ & $* * *$ & $<0.001$ & $4.05(2.39-6.18)$ & $* * *$ & $<0.001$ \\
\hline DE0732A & $-0.02(-0.21-0.12)$ & & 0.703 & $0.45(-0.90-2.19)$ & & 0.531 \\
\hline DE0735A & $0.33(0.15-0.51)$ & $* * *$ & $<0.001$ & $3.62(1.98-5.51)$ & $* * *$ & $<0.001$ \\
\hline DE0737A & $0.19(-0.05-0.42)$ & + & 0.078 & $1.43(-0.15-3.28)$ & * & 0.049 \\
\hline DE0738A & $0.10(0.04-0.15)$ & $* * *$ & $<0.001$ & $3.67(1.64-5.62)$ & $* * *$ & $<0.001$ \\
\hline DE0739A & $0.32(0.11-0.53)$ & $* *$ & 0.001 & $2.32(0.29-4.58)$ & $*$ & 0.016 \\
\hline DE0754A & $0.00(-0.06-0.03)$ & & 0.799 & $-0.43(-5.03-1.81)$ & & 0.543 \\
\hline DE0844A & $0.07(0.00-0.17)$ & $*$ & 0.047 & $0.48(0.02-1.47)$ & $*$ & 0.026 \\
\hline DE0874A & $0.00(0.00-0.01)$ & $* * *$ & $<0.001$ & $0.00(0.00-0.00)$ & $*$ & 0.017 \\
\hline DE0907A & $0.01(0.00-0.09)$ & & 0.193 & $0.67(0.01-2.28)$ & $*$ & 0.013 \\
\hline DE0960A & $0.00(-0.18-0.19)$ & & 0.943 & $-0.04(-1.68-1.88)$ & & 0.965 \\
\hline DE0996A & $0.26(0.12-0.42)$ & $* *$ & 0.004 & $1.51(0.75-2.40)$ & $* * *$ & $<0.001$ \\
\hline EE0011R & $-0.38(-0.62--0.10)$ & $* *$ & 0.004 & $-1.54(-2.69--0.10)$ & $*$ & 0.014 \\
\hline${ }^{\dagger} \mathrm{ES} 0007 \mathrm{R}$ & $-0.62(-1.16--0.14)$ & $* *$ & 0.007 & $-2.39(-4.47--0.54)$ & $* *$ & 0.005 \\
\hline ES1222A & $-0.11(-0.18--0.05)$ & $* *$ & 0.007 & $-4.88(-7.44--2.83)$ & $* * *$ & $<0.001$ \\
\hline ES1400A & $-0.69(-0.98--0.38)$ & $* * *$ & $<0.001$ & $-5.14(-7.61--2.94)$ & $* * *$ & $<0.001$ \\
\hline${ }^{\dagger} \mathrm{ES} 1435 \mathrm{~A}$ & $0.11(-0.14-0.32)$ & & 0.345 & $0.43(-0.67-1.27)$ & & 0.370 \\
\hline${ }^{\dagger} \mathrm{ES} 1437 \mathrm{~A}$ & $-0.49(-0.72--0.20)$ & $* * *$ & 0.001 & $-1.70(-2.51--0.76)$ & $* * *$ & $<0.001$ \\
\hline${ }^{\dagger} \mathrm{ES} 1441 \mathrm{~A}$ & $-0.11(-0.37-0.14)$ & & 0.421 & $-0.37(-1.25-0.30)$ & & 0.310 \\
\hline FR08 & $0.27(-0.07-0.62)$ & + & 0.085 & $1.45(-0.44-3.73)$ & + & 0.099 \\
\hline GB0002R & $0.04(-0.07-0.19)$ & & 0.407 & $0.87(-1.04-2.98)$ & & 0.329 \\
\hline GB0006R & $0.26(0.11-0.40)$ & $* * *$ & $<0.001$ & $6.92(3.19-10.08)$ & $* * *$ & $<0.001$ \\
\hline GB0013R & $0.22(-0.01-0.45)$ & + & 0.060 & $1.54(-0.16-3.45)$ & & 0.102 \\
\hline GB0014R & $0.32(0.17-0.50)$ & $* * *$ & $<0.001$ & $3.34(1.74-5.55)$ & $* * *$ & $<0.001$ \\
\hline GB0015R & $0.08(-0.13-0.36)$ & & 0.427 & $0.38(-0.68-1.62)$ & & 0.451 \\
\hline GB0031R & $0.53(0.16-0.86)$ & $* *$ & 0.003 & $3.27(1.04-5.55)$ & $* *$ & 0.002 \\
\hline GB0033R & $0.17(-0.05-0.43)$ & & 0.174 & $1.86(0.00-3.89)$ & + & 0.082 \\
\hline GB0036R & $0.24(0.07-0.44)$ & $* *$ & 0.003 & $2.86(0.30-5.54)$ & $*$ & 0.024 \\
\hline GB0037R & $0.26(0.03-0.46)$ & $*$ & 0.024 & $2.50(0.62-4.89)$ & $*$ & 0.010 \\
\hline GB0038R & $0.13(-0.03-0.30)$ & + & 0.100 & $1.54(-0.27-3.36)$ & & 0.105 \\
\hline GB0039R & $0.25(0.10-0.40)$ & $* *$ & 0.002 & $4.35(2.21-7.14)$ & $* * *$ & $<0.001$ \\
\hline GB0044R & $0.15(-0.04-0.33)$ & & 0.106 & $1.30(-0.57-3.22)$ & & 0.187 \\
\hline
\end{tabular}


Table A3: continued. Quantification of trends (ppbv/yr and \%/yr) in $\mathrm{O}_{3}$ monthly $5^{\text {th }}$ percentiles 1996-2005. 95\% confidence interval given in brackets. *** significant at $99.9 \%$ level, ${ }^{* *}$ significant at $99.0 \%$ level, $*$ significant at $95.0 \%$ level, + significant at $90.0 \%$ level. ${ }^{\dagger}$ high altitude sites $(>1000 \mathrm{~m}) .{ }^{\ddagger} 2 \sigma$ error. ${ }^{\diamond}$ significant trends only

\begin{tabular}{|c|c|c|c|c|c|c|}
\hline ID & Trend (ppbv/yr) & Sig. & $\mathrm{p}$ value & Trend $(\% / y r)$ & Sig & $\mathrm{p}$ value \\
\hline GB0045R & $0.38(0.26-0.50)$ & $* * *$ & $<0.001$ & $10.64(7.67-13.97)$ & $* * *$ & $<0.001$ \\
\hline GB0617A & $0.04(-0.02-0.14)$ & & 0.206 & $1.63(0.04-4.53)$ & + & 0.053 \\
\hline HPB & $0.16(-0.09-0.41)$ & & 0.201 & $1.01(-0.21-2.28)$ & + & 0.077 \\
\hline HU0002R & $-0.70(-1.00--0.39)$ & $* * *$ & $<0.001$ & $-4.87(-6.93--2.74)$ & $* * *$ & $<0.001$ \\
\hline IE31 & $0.19(-0.16-0.56)$ & & 0.228 & $0.48(-0.93-2.35)$ & & 0.457 \\
\hline IT04 & $0.67(0.52-0.80)$ & $* * *$ & $<0.001$ & $20.02(16.91-23.46)$ & $* * *$ & $<0.001$ \\
\hline LT0015R & $0.36(0.05-0.65)$ & $*$ & 0.023 & $2.40(-0.08-4.57)$ & + & 0.053 \\
\hline LV0010R & $-0.45(-0.59--0.29)$ & $* * *$ & $<0.001$ & $-5.31(-7.71--3.29)$ & $* * *$ & $<0.001$ \\
\hline NL0007R & $0.02(-0.04-0.09)$ & & 0.571 & $-0.68(-4.44-2.93)$ & & 0.660 \\
\hline NL0009R & $0.08(0.02-0.19)$ & $*$ & 0.022 & $2.92(1.08-4.90)$ & $* *$ & 0.004 \\
\hline NL0010R & $-0.01(-0.05-0.04)$ & & 0.468 & $-2.39(-5.94-0.63)$ & + & 0.086 \\
\hline NL0196A & $-0.03(-0.08-0.00)$ & + & 0.062 & $-4.83(-8.63--0.63)$ & $*$ & 0.017 \\
\hline NL0198A & $0.00(-0.06-0.08)$ & & 0.894 & $-0.27(-3.19-2.81)$ & & 0.848 \\
\hline NL0202A & $0.04(-0.01-0.09)$ & & 0.117 & $1.85(-2.20-5.83)$ & & 0.347 \\
\hline NL0205A & $-0.05(-0.13-0.03)$ & & 0.223 & $-2.76(-6.51-0.74)$ & & 0.108 \\
\hline NL0207A & $0.15(0.03-0.29)$ & $* *$ & 0.008 & $2.19(0.18-4.83)$ & + & 0.052 \\
\hline NL0209A & $0.03(-0.03-0.11)$ & & 0.345 & $3.13(-2.44-8.79)$ & & 0.197 \\
\hline NL0220A & $0.03(-0.02-0.11)$ & & 0.198 & $0.98(-1.80-4.14)$ & & 0.508 \\
\hline NL0223A & $0.09(0.04-0.15)$ & $* *$ & 0.001 & $6.16(2.87-9.35)$ & $* *$ & 0.002 \\
\hline NL0226A & $-0.03(-0.07-0.01)$ & & 0.197 & $-3.94(-8.38-0.25)$ & + & 0.093 \\
\hline NL0227A & $0.00(-0.06-0.11)$ & & 0.895 & $-2.50(-5.76-1.06)$ & & 0.165 \\
\hline NL0228A & $0.03(-0.02-0.09)$ & & 0.249 & $0.92(-2.04-4.24)$ & & 0.465 \\
\hline NL0229A & $0.04(0.01-0.10)$ & $*$ & 0.013 & $4.28(0.73-7.71)$ & $*$ & 0.012 \\
\hline NL0231A & $0.03(-0.05-0.15)$ & & 0.432 & $0.85(-3.03-4.48)$ & & 0.610 \\
\hline NL0232A & $-0.02(-0.04-0.01)$ & & 0.199 & $-2.79(-5.26-0.10)$ & + & 0.066 \\
\hline NL0250A & $-0.06(-0.12--0.01)$ & $*$ & 0.023 & $-3.65(-6.48-0.28)$ & + & 0.059 \\
\hline NO01 & $-0.26(-0.42--0.07)$ & $* *$ & 0.003 & $-3.33(-5.42--1.04)$ & $* *$ & 0.002 \\
\hline${ }^{\dagger} \mathrm{PLO} 03$ & $-0.88(-1.51--0.37)$ & $* * *$ & $<0.001$ & $-4.11(-7.53--1.40)$ & $* *$ & 0.002 \\
\hline PT0004R & $-0.39(-0.93-0.08)$ & $*$ & 0.042 & $-3.82(-9.33-1.70)$ & + & 0.084 \\
\hline${ }^{\dagger} \mathrm{PUY}$ & $0.13(-0.18-0.46)$ & & 0.435 & $0.43(-0.51-1.51)$ & & 0.374 \\
\hline SI0008R & $-0.05(-0.09-0.02)$ & & 0.116 & $-4.90(-8.75--0.75)$ & $*$ & 0.011 \\
\hline †ZUG & $-0.34(-0.55--0.15)$ & $* *$ & 0.003 & $-0.90(-1.39--0.43)$ & $* *$ & 0.002 \\
\hline $\begin{array}{c}\text { European } \\
\text { average }^{\ddagger}\end{array}$ & $0.13 \pm 0.02 \mathrm{ppbv} / \mathrm{yr}$ & & & $1.40 \pm 0.19 \% / \mathrm{yr}$ & & \\
\hline Range $^{\diamond}$ & -0.88 to $0.98 \mathrm{ppbv} / \mathrm{yr}$ & & & -6.32 to $20.02 \% / y r$ & & \\
\hline
\end{tabular}


Table A4: Quantification of trends (ppbv/yr and \%/yr) in $\mathrm{O}_{3}$ monthly $95^{\text {th }}$ percentiles 1996-2005. 95\% confidence interval given in brackets. $* * *$ significant at $99.9 \%$ level, ** significant at $99.0 \%$ level, ${ }^{*}$ significant at $95.0 \%$ level, + significant at $90.0 \%$ level. $\dagger$ high altitude sites $(>1000 \mathrm{~m})$. ${ }^{\ddagger} 2 \sigma$ error. ${ }^{\diamond}$ significant trends only

\begin{tabular}{|c|c|c|c|c|c|c|}
\hline ID & Trend (ppbv/yr) & Sig. & $\mathrm{p}$ value & Trend $(\% / y r)$ & Sig & $\mathrm{p}$ value \\
\hline AT0002R & $0.23(-0.02-0.49)$ & + & 0.074 & $0.50(-0.06-1.00)$ & + & 0.075 \\
\hline${ }^{\dagger} \mathrm{AT} 0004 \mathrm{R}$ & $0.25(-0.01-0.55)$ & $*$ & 0.046 & $0.44(-0.04-0.99)$ & + & 0.058 \\
\hline${ }^{\dagger} \mathrm{AT} 0005 \mathrm{R}$ & $0.35(0.07-0.62)$ & $*$ & 0.021 & $0.69(0.15-1.18)$ & $*$ & 0.018 \\
\hline${ }^{\dagger} \mathrm{AT} 0034 \mathrm{R}$ & $0.45(0.20-0.69)$ & $* * *$ & $<0.001$ & $0.91(0.41-1.47)$ & $* * *$ & $<0.001$ \\
\hline AT0044A & $-0.06(-0.40-0.30)$ & & 0.810 & $-0.16(-0.92-0.75)$ & & 0.686 \\
\hline AT0052A & $0.31(-0.03-0.66)$ & + & 0.093 & $0.59(-0.04-1.25)$ & + & 0.089 \\
\hline AT0054A & $0.25(0.01-0.53)$ & $*$ & 0.046 & $0.41(-0.05-1.01)$ & + & 0.083 \\
\hline${ }^{\dagger} \mathrm{AT} 0058 \mathrm{~A}$ & $0.19(-0.04-0.48)$ & + & 0.070 & $0.37(-0.05-0.84)$ & + & 0.061 \\
\hline${ }^{\dagger} \mathrm{AT} 0064 \mathrm{~A}$ & $0.25(0.02-0.50)$ & $*$ & 0.033 & $0.44(-0.03-0.93)$ & + & 0.060 \\
\hline AT0069A & $0.22(-0.07-0.50)$ & & 0.132 & $0.40(-0.14-0.94)$ & & 0.148 \\
\hline AT0073A & $0.00(-0.29-0.29)$ & & 0.971 & $-0.12(-0.67-0.36)$ & & 0.686 \\
\hline${ }^{\dagger}$ AT0079A & $0.22(0.00-0.40)$ & $*$ & 0.031 & $0.36(-0.01-0.66)$ & $*$ & 0.031 \\
\hline AT0080A & $0.11(-0.29-0.44)$ & & 0.537 & $0.19(-0.76-0.98)$ & & 0.651 \\
\hline AT0086A & $0.79(0.39-1.17)$ & $* * *$ & $<0.001$ & $1.75(1.00-2.71)$ & $* * *$ & $<0.001$ \\
\hline${ }^{\dagger}$ AT0089A & $0.19(0.01-0.43)$ & $*$ & 0.030 & $0.49(0.12-0.88)$ & $* *$ & 0.007 \\
\hline AT0094A & $0.21(-0.15-0.68)$ & & 0.264 & $0.36(-0.35-1.19)$ & & 0.316 \\
\hline AT0095A & $0.75(0.44-1.08)$ & $* * *$ & $<0.001$ & $1.77(1.10-2.47)$ & $* * *$ & $<0.001$ \\
\hline AT0096A & $0.56(0.21-0.96)$ & $* *$ & 0.003 & $1.12(0.41-1.89)$ & $* *$ & 0.004 \\
\hline AT0101A & $0.32(0.02-0.62)$ & $*$ & 0.035 & $0.63(0.07-1.23)$ & $*$ & 0.032 \\
\hline AT0102A & $0.63(0.31-0.93)$ & $* * *$ & $<0.001$ & $1.24(0.52-2.00)$ & $* *$ & 0.002 \\
\hline AT0103A & $-0.01(-0.30-0.30)$ & & 0.935 & $-0.03(-0.59-0.62)$ & & 0.921 \\
\hline AT0105A & $0.64(0.31-1.01)$ & $* * *$ & $<0.001$ & $1.28(0.52-1.98)$ & $* * *$ & $<0.001$ \\
\hline${ }^{\dagger} \mathrm{AT} 0108 \mathrm{~A}$ & $0.33(-0.04-0.70)$ & + & 0.053 & $0.57(-0.09-1.19)$ & $*$ & 0.046 \\
\hline AT0111A & $0.68(0.32-1.02)$ & $* * *$ & $<0.001$ & $1.44(0.56-2.24)$ & $* *$ & 0.001 \\
\hline AT0115A & $0.18(-0.13-0.50)$ & & 0.198 & $0.35(-0.26-0.99)$ & & 0.196 \\
\hline AT0121A & $0.45(0.20-0.69)$ & $* * *$ & $<0.001$ & $0.91(0.41-1.47)$ & $* * *$ & $<0.001$ \\
\hline AT0122A & $0.31(-0.11-0.73)$ & & 0.127 & $0.59(-0.47-1.50)$ & & 0.231 \\
\hline${ }^{\dagger} \mathrm{AT} 0124 \mathrm{~A}$ & $-0.02(-0.30-0.34)$ & & 0.881 & $-0.01(-0.53-0.60)$ & & 0.967 \\
\hline AT0128A & $0.31(-0.07-0.66)$ & + & 0.094 & $0.65(-0.12-1.50)$ & + & 0.078 \\
\hline AT0134A & $0.76(0.43-1.18)$ & $* * *$ & $<0.001$ & $1.55(0.87-2.38)$ & $* * *$ & $<0.001$ \\
\hline AT0141A & $0.33(0.10-0.59)$ & $*$ & 0.010 & $0.69(0.17-1.31)$ & $*$ & 0.012 \\
\hline AT0143A & $0.52(0.21-0.82)$ & $* * *$ & $<0.001$ & $1.31(0.52-2.18)$ & $* * *$ & $<0.001$ \\
\hline${ }^{\dagger} \mathrm{AT} 0146 \mathrm{~A}$ & $0.14(-0.07-0.36)$ & & 0.157 & $0.28(-0.17-0.72)$ & & 0.181 \\
\hline AT0149A & $0.56(0.30-0.81)$ & $* * *$ & $<0.001$ & $1.18(0.67-1.68)$ & $* * *$ & $<0.001$ \\
\hline AT0153A & $0.13(-0.20-0.51)$ & & 0.457 & $0.27(-0.37-1.00)$ & & 0.430 \\
\hline AT0154A & $1.16(0.79-1.52)$ & $* * *$ & $<0.001$ & $2.06(1.39-2.74)$ & $* * *$ & $<0.001$ \\
\hline AT0162A & $0.24(-0.07-0.56)$ & + & 0.097 & $0.52(-0.21-1.29)$ & & 0.144 \\
\hline AT0164A & $0.45(0.01-0.85)$ & $*$ & 0.018 & $0.82(0.08-1.57)$ & $*$ & 0.012 \\
\hline AT0166A & $0.73(0.48-1.02)$ & $* * *$ & $<0.001$ & $1.61(0.94-2.34)$ & $* * *$ & $<0.001$ \\
\hline AT0167A & $1.21(0.79-1.67)$ & $* * *$ & $<0.001$ & $2.04(1.30-2.94)$ & $* * *$ & $<0.001$ \\
\hline AT0175A & $0.41(0.05-0.79)$ & $*$ & 0.012 & $0.74(0.06-1.35)$ & $*$ & 0.019 \\
\hline AT0176A & $0.48(0.17-0.76)$ & $* *$ & 0.002 & $0.81(0.24-1.37)$ & $* *$ & 0.004 \\
\hline
\end{tabular}


Table A4: continued. Quantification of trends (ppbv/yr and \%/yr) in $\mathrm{O}_{3}$ monthly $95^{\text {th }}$ percentiles 1996-2005. 95\% confidence interval given in brackets. *** significant at 99.9\% level, ${ }^{* *}$ significant at $99.0 \%$ level, ${ }^{*}$ significant at $95.0 \%$ level, + significant at $90.0 \%$ level. ${ }^{\dagger}$ high altitude sites $(>1000 \mathrm{~m}) .{ }^{\ddagger} 2 \sigma$ error. ${ }^{\diamond}$ significant trends only

\begin{tabular}{|c|c|c|c|c|c|c|}
\hline ID & Trend (ppbv/yr) & Sig. & $\mathrm{p}$ value & Trend $(\% / y r)$ & Sig & $\mathrm{p}$ value \\
\hline${ }^{\dagger}$ AT0180A & $-0.37(-0.71-0.06)$ & $*$ & 0.031 & $-0.62(-1.23-0.10)$ & $*$ & 0.025 \\
\hline BE0032R & $0.29(-0.08-0.69)$ & & 0.124 & $0.66(-0.15-1.53)$ & + & 0.098 \\
\hline BE0033R & $-0.28(-0.56-0.01)$ & * & 0.031 & $-0.72(-1.36--0.07)$ & $*$ & 0.023 \\
\hline BE0035R & $0.21(-0.09-0.55)$ & & 0.221 & $0.57(-0.03-1.25)$ & + & 0.086 \\
\hline BE0211A & $0.17(-0.21-0.60)$ & & 0.390 & $0.14(-0.73-1.23)$ & & 0.710 \\
\hline BE0238A & $0.30(0.01-0.61)$ & + & 0.053 & $0.61(0.01-1.25)$ & + & 0.053 \\
\hline BE0294A & $-0.02(-0.46-0.50)$ & & 0.913 & $0.08(-0.90-1.08)$ & & 0.872 \\
\hline BE0298A & $0.40(-0.07-0.87)$ & + & 0.064 & $0.84(-0.11-1.90)$ & $*$ & 0.048 \\
\hline BE0302A & $0.46(0.19-0.73)$ & $* *$ & 0.003 & $1.03(0.42-1.64)$ & $* *$ & 0.002 \\
\hline BE0304A & $0.48(0.09-0.87)$ & $*$ & 0.014 & $1.05(0.28-1.82)$ & $*$ & 0.013 \\
\hline BE0311A & $-0.11(-0.45-0.21)$ & & 0.500 & $-0.22(-0.90-0.49)$ & & 0.506 \\
\hline BE0345A & $-0.46(-0.88-0.05)$ & $*$ & 0.043 & $-0.84(-1.83-0.08)$ & $*$ & 0.049 \\
\hline${ }^{\dagger} \mathrm{CH} 0001 \mathrm{R}$ & $0.15(-0.01-0.28)$ & + & 0.072 & $0.24(-0.02-0.48)$ & + & 0.068 \\
\hline $\mathrm{CH} 0002 \mathrm{R}$ & $0.25(-0.04-0.58)$ & + & 0.072 & $0.43(-0.13-1.05)$ & & 0.107 \\
\hline CH0003R & $0.04(-0.24-0.36)$ & & 0.831 & $0.06(-0.50-0.77)$ & & 0.856 \\
\hline${ }^{\dagger} \mathrm{CH} 0004 \mathrm{R}$ & $0.00(-0.29-0.30)$ & & 0.993 & $-0.12(-0.62-0.39)$ & & 0.631 \\
\hline${ }^{\dagger} \mathrm{CH} 0005 \mathrm{R}$ & $0.13(-0.10-0.38)$ & & 0.318 & $0.16(-0.22-0.57)$ & & 0.457 \\
\hline CH0019A & $0.10(-0.59-0.60)$ & & 0.653 & $0.13(-1.05-1.03)$ & & 0.768 \\
\hline CH0024A & $0.02(-0.30-0.35)$ & & 03 & $0.03(-0.65-0.75)$ & & 0.892 \\
\hline CH0033A & $-0.40(-0.76--0.02)$ & $*$ & 0.041 & $-0.72(-1.40-0.03)$ & + & 0.051 \\
\hline${ }^{\dagger} \mathrm{CMN}$ & $0.14(-0.26-0.42)$ & & 0.471 & $0.17(-0.42-0.60)$ & & 0.462 \\
\hline CZ0001R & $-0.30(-0.60-0.02)$ & $*$ & 0.049 & $-0.62(-1.23-0.04)$ & + & 0.053 \\
\hline CZ0003R & $0.05(-0.22-0.33)$ & & 0.696 & $0.10(-0.44-0.69)$ & & 0.634 \\
\hline CZ0017A & $-0.24(-0.51-0.07)$ & & 0.111 & $-0.44(-0.98-0.11)$ & & 0.100 \\
\hline CZ0030A & $0.22(-0.13-0.66)$ & & 0.144 & $0.54(-0.29-1.40)$ & & 0.149 \\
\hline CZ0041A & $0.51(-0.01-1.09)$ & $*$ & 0.038 & $1.06(-0.21-2.21)$ & + & 0.053 \\
\hline CZ0045A & $0.47(0.15-0.85)$ & $* *$ & 0.003 & $0.96(0.34-1.66)$ & $* *$ & 0.002 \\
\hline${ }^{\dagger} \mathrm{CZ} 0049 \mathrm{~A}$ & $-0.23(-0.49-0.05)$ & & 0.112 & $-0.46(-0.91-0.05)$ & + & 0.085 \\
\hline CZ0051A & $0.16(-0.13-0.48)$ & & 0.266 & $0.32(-0.23-0.96)$ & & 0.236 \\
\hline${ }^{\dagger} \mathrm{CZ} 0055 \mathrm{~A}$ & $-0.61(-1.27-0.10)$ & $*$ & 0.044 & $-1.17(-2.32-0.12)$ & $*$ & 0.034 \\
\hline CZ0057A & $0.03(-0.37-0.40)$ & & 0.906 & $0.01(-0.81-0.68)$ & & 0.975 \\
\hline $\mathrm{CZ} 0062 \mathrm{~A}$ & $0.49(0.21-0.85)$ & $* *$ & 0.001 & $0.80(0.23-1.53)$ & $* *$ & 0.007 \\
\hline${ }^{\dagger} \mathrm{DE} 0003 \mathrm{R}$ & $0.42(0.03-0.78)$ & $*$ & 0.031 & $0.58(0.03-1.14)$ & $*$ & 0.043 \\
\hline DE0007R & $0.36(0.05-0.69)$ & + & 0.052 & $0.79(0.11-1.49)$ & + & 0.052 \\
\hline DE0008R & $0.26(-0.07-0.64)$ & & 0.101 & $0.46(-0.20-1.16)$ & & 0.178 \\
\hline DE0009R & $0.14(-0.14-0.41)$ & & 0.334 & $0.35(-0.28-0.97)$ & & 0.288 \\
\hline DE0035R & $0.39(-0.02-0.81)$ & * & 0.041 & $0.88(0.05-1.70)$ & $*$ & 0.026 \\
\hline DE0422A & $0.81(0.47-1.15)$ & $* * *$ & $<0.001$ & $1.74(1.00-2.48)$ & $* * *$ & $<0.001$ \\
\hline DE0510A & $0.26(-0.04-0.63)$ & & 0.114 & $0.51(-0.26-1.32)$ & & 0.168 \\
\hline DE0514A & $0.10(-0.19-0.46)$ & & 0.528 & $0.21(-0.45-0.99)$ & & 0.555 \\
\hline DE0556A & $0.51(0.24-0.86)$ & $* * *$ & $<0.001$ & $1.02(0.54-1.65)$ & $* * *$ & $<0.001$ \\
\hline DE0649A & $0.59(0.28-0.90)$ & $* * *$ & $<0.001$ & $1.33(0.70-1.98)$ & $* * *$ & $<0.001$ \\
\hline
\end{tabular}


Table A4: continued. Quantification of trends (ppbv/yr and \%/yr) in $\mathrm{O}_{3}$ monthly $95^{\text {th }}$ percentiles 1996-2005. 95\% confidence interval given in brackets. *** significant at 99.9\% level, ${ }^{* *}$ significant at $99.0 \%$ level, ${ }^{*}$ significant at $95.0 \%$ level, + significant at $90.0 \%$ level. ${ }^{\dagger}$ high altitude sites $(>1000 \mathrm{~m}) . \stackrel{\ddagger}{ } 2 \sigma$ error. ${ }^{\diamond}$ significant trends only

\begin{tabular}{|c|c|c|c|c|c|c|}
\hline ID & Trend (ppbv/yr) & Sig. & $\mathrm{p}$ value & Trend $(\% / y r)$ & Sig & $\mathrm{p}$ value \\
\hline DE0651A & $0.40(0.12-0.77)$ & $*$ & 0.014 & $0.73(0.12-1.42)$ & $*$ & 0.033 \\
\hline DE0674A & $0.00(-0.24-0.25)$ & & 0.967 & $-0.02(-0.50-0.52)$ & & 0.928 \\
\hline DE0679A & $-0.03(-0.31-0.28)$ & & 0.835 & $-0.19(-0.74-0.36)$ & & 0.496 \\
\hline DE0680A & $0.54(0.25-0.87)$ & $* * *$ & $<0.001$ & $1.21(0.55-1.89)$ & $* * *$ & $<0.001$ \\
\hline DE0684A & $-0.44(-1.06-0.15)$ & & 0.169 & $-0.77(-1.75-0.20)$ & & 0.121 \\
\hline DE0685A & $0.40(0.13-0.75)$ & $* *$ & 0.002 & $0.82(0.24-1.52)$ & $* *$ & 0.004 \\
\hline DE0686A & $-0.11(-0.35-0.21)$ & & 0.394 & $-0.23(-0.75-0.43)$ & & 0.485 \\
\hline DE0687A & $0.10(-0.17-0.39)$ & & 0.488 & $0.21(-0.39-0.80)$ & & 0.519 \\
\hline DE0688A & $-0.05(-0.30-0.22)$ & & 0.680 & $-0.14(-0.72-0.56)$ & & 0.657 \\
\hline DE0699A & $0.64(0.33-0.96)$ & $* * *$ & $<0.001$ & $1.22(0.63-1.82)$ & $* * *$ & $<0.001$ \\
\hline DE0719A & $0.57(0.25-0.93)$ & $* *$ & 0.002 & $1.10(0.49-1.80)$ & $* *$ & 0.001 \\
\hline DE0732A & $0.20(-0.09-0.52)$ & & 0.166 & $0.44(-0.24-1.11)$ & & 0.187 \\
\hline DE0735A & $0.40(0.14-0.71)$ & $* *$ & 0.004 & $0.91(0.38-1.47)$ & $* *$ & 0.003 \\
\hline DE0737A & $-0.39(-0.67--0.03)$ & $*$ & 0.023 & $-0.88(-1.42--0.25)$ & $* *$ & 0.006 \\
\hline DE0738A & $0.20(-0.11-0.55)$ & & 0.198 & $0.35(-0.28-1.10)$ & & 0.295 \\
\hline DE0739A & $0.25(-0.07-0.60)$ & + & 0.100 & $0.37(-0.29-1.13)$ & & 0.247 \\
\hline DE0754A & $-0.10(-0.43-0.22)$ & & 0.519 & $-0.27(-1.10-0.56)$ & & 0.488 \\
\hline DE0844A & $0.03(-0.22-0.26)$ & & 0.810 & $0.02(-0.60-0.59)$ & & 0.949 \\
\hline DE0874A & $0.28(0.05-0.55)$ & $*$ & 0.022 & $0.50(-0.06-1.18)$ & + & 0.078 \\
\hline DE0907A & $0.51(0.10-0.99)$ & * & 0.040 & $1.31(0.28-2.34)$ & $*$ & 0.022 \\
\hline DE0960A & $0.16(-0.18-0.50)$ & & 0.341 & $0.37(-0.40-1.07)$ & & 0.341 \\
\hline DE0996A & $0.23(-0.18-0.65)$ & & 0.196 & $0.48(-0.27-1.29)$ & & 0.175 \\
\hline EE0011R & $-0.51(-0.76--0.23)$ & $* * *$ & $<0.001$ & $-1.11(-1.72--0.48)$ & $* * *$ & $<0.001$ \\
\hline${ }^{\dagger} \mathrm{ES} 0007 \mathrm{R}$ & $-0.75(-1.36--0.15)$ & $* *$ & 0.006 & $-1.38(-2.54--0.28)$ & $* *$ & 0.005 \\
\hline ES1222A & $-0.63(-0.85--0.40)$ & $* * *$ & $<0.001$ & $-1.39(-1.96--0.88)$ & $* * *$ & $<0.001$ \\
\hline ES1400A & $-1.41(-1.86--0.97)$ & $* * *$ & $<0.001$ & $-2.48(-3.23--1.74)$ & $* * *$ & $<0.001$ \\
\hline${ }^{\dagger} \mathrm{ES} 1435 \mathrm{~A}$ & $0.34(0.08-0.65)$ & $*$ & 0.023 & $0.49(0.02-1.04)$ & + & 0.060 \\
\hline${ }^{\dagger} \mathrm{ES} 1437 \mathrm{~A}$ & $-0.65(-0.96--0.29)$ & $* * *$ & $<0.001$ & $-1.28(-1.93--0.67)$ & $* * *$ & $<0.001$ \\
\hline${ }^{\dagger} \mathrm{ES} 1441 \mathrm{~A}$ & $-0.19(-0.44-0.04)$ & & 0.184 & $-0.38(-0.84-0.00)$ & & 0.100 \\
\hline FR08 & $0.25(-0.18-0.68)$ & & 0.253 & $0.42(-0.42-1.24)$ & & 0.310 \\
\hline GB0002R & $-0.40(-0.60--0.22)$ & $* * *$ & $<0.001$ & $-1.03(-1.55--0.57)$ & $* * *$ & $<0.001$ \\
\hline GB0006R & $0.14(-0.13-0.41)$ & & 0.246 & $0.29(-0.40-1.02)$ & & 0.352 \\
\hline GB0013R & $0.18(-0.03-0.39)$ & + & 0.100 & $0.47(-0.02-0.94)$ & + & 0.066 \\
\hline GB0014R & $0.20(-0.03-0.41)$ & & 0.110 & $0.47(-0.07-0.97)$ & & 0.120 \\
\hline GB0015R & $0.22(-0.02-0.41)$ & + & 0.070 & $0.46(-0.12-0.88)$ & + & 0.098 \\
\hline GB0031R & $0.39(0.09-0.64)$ & $* *$ & 0.002 & $0.90(0.24-1.45)$ & $* *$ & 0.002 \\
\hline GB0033R & $-0.04(-0.23-0.19)$ & & 0.525 & $-0.11(-0.59-0.47)$ & & 0.707 \\
\hline GB0036R & $0.20(-0.05-0.49)$ & & 0.115 & $0.45(-0.24-1.21)$ & & 0.157 \\
\hline GB0037R & $0.08(-0.29-0.41)$ & & 0.657 & $0.16(-0.77-1.00)$ & & 0.686 \\
\hline GB0038R & $0.18(-0.17-0.52)$ & & 0.293 & $0.33(-0.32-1.05)$ & & 0.323 \\
\hline GB0039R & $0.32(0.00-0.61)$ & * & 0.025 & $0.81(0.08-1.51)$ & $*$ & 0.015 \\
\hline GB0044R & $-0.21(-0.45-0.00)$ & $*$ & 0.044 & $-0.44(-1.02-0.01)$ & + & 0.065 \\
\hline
\end{tabular}


Table A4: continued. Quantification of trends (ppbv/yr and \%/yr) in $\mathrm{O}_{3}$ monthly $95^{\text {th }}$ percentiles 1996-2005. 95\% confidence interval given in brackets. *** significant at $99.9 \%$ level, ${ }^{* *}$ significant at $99.0 \%$ level, ${ }^{*}$ significant at $95.0 \%$ level, + significant at $90.0 \%$ level. ${ }^{\dagger}$ high altitude sites $(>1000 \mathrm{~m}) .{ }^{\ddagger} 2 \sigma$ error. ${ }^{\diamond}$ significant trends only

\begin{tabular}{|c|c|c|c|c|c|c|}
\hline ID & Trend (ppbv/yr) & Sig. & $p$ value & Trend (\%/yr) & Sig & $\mathrm{p}$ value \\
\hline GB0045R & $0.76(0.50-1.02)$ & $* * *$ & $<0.001$ & $2.09(1.36-2.76)$ & $* * *$ & $<0.001$ \\
\hline GB0617A & $-0.06(-0.30-0.16)$ & & 0.546 & $-0.14(-0.67-0.38)$ & & 0.654 \\
\hline HPB & $0.23(0.00-0.51)$ & + & 0.078 & $0.32(-0.05-0.72)$ & & 0.122 \\
\hline HU0002R & $-1.62(-2.23--1.09)$ & $* * *$ & $<0.001$ & $-3.12(-4.05--2.07)$ & $* * *$ & $<0.001$ \\
\hline IE31 & $-0.05(-0.23-0.12)$ & & 0.525 & $-0.11(-0.52-0.24)$ & & 0.502 \\
\hline IT04 & $-0.57(-1.17-0.07)$ & $*$ & 0.045 & $-0.75(-1.88-0.55)$ & & 0.226 \\
\hline LT0015R & $0.26(-0.08-0.54)$ & + & 0.063 & $0.55(-0.24-1.25)$ & + & 0.081 \\
\hline LV0010R & $-0.32(-0.62--0.04)$ & $*$ & 0.040 & $-0.86(-1.58--0.05)$ & $*$ & 0.042 \\
\hline NL0007R & $0.12(-0.19-0.46)$ & & 0.457 & $0.41(-0.39-1.29)$ & & 0.367 \\
\hline NL0009R & $0.50(0.25-0.71)$ & $* * *$ & $<0.001$ & $1.16(0.52-1.69)$ & $* * *$ & $<0.001$ \\
\hline NL0010R & $0.32(0.01-0.65)$ & $*$ & 0.039 & $0.77(-0.02-1.62)$ & $*$ & 0.045 \\
\hline NL0196A & $0.13(-0.16-0.46)$ & & 0.393 & $0.34(-0.33-1.12)$ & & 0.361 \\
\hline NL0198A & $0.15(-0.18-0.49)$ & & 0.420 & $0.36(-0.39-1.21)$ & & 0.361 \\
\hline NL0202A & $0.24(-0.07-0.58)$ & & 0.122 & $0.38(-0.47-1.23)$ & & 0.345 \\
\hline NL0205A & $0.46(0.09-0.85)$ & $*$ & 0.014 & $1.11(0.18-2.01)$ & $*$ & 0.023 \\
\hline NL0207A & $0.14(-0.15-0.41)$ & & 0.353 & $0.25(-0.29-0.95)$ & & 0.487 \\
\hline NL0209A & $0.24(-0.12-0.59)$ & & 0.167 & $0.70(-0.27-1.60)$ & & 0.153 \\
\hline NL0220A & $-0.04(-0.32-0.32)$ & & 0.761 & $-0.11(-0.79-0.82)$ & & 0.741 \\
\hline NL0223A & $-0.01(-0.42-0.38)$ & & 0.973 & $0.10(-0.84-1.01)$ & & 0.842 \\
\hline NL0226A & $0.30(0.00-0.57)$ & + & 0.053 & $0.78(-0.01-1.49)$ & + & 0.053 \\
\hline NL0227A & $-0.05(-0.24-0.17)$ & & 0.650 & $-0.17(-0.65-0.38)$ & & 0.525 \\
\hline NL0228A & $0.34(0.06-0.63)$ & $*$ & 0.017 & $0.78(0.07-1.49)$ & $*$ & 0.027 \\
\hline NL0229A & $0.51(0.23-0.83)$ & $* *$ & 0.001 & $1.44(0.66-2.30)$ & $* * *$ & $<0.001$ \\
\hline NL0231A & $0.01(-0.38-0.40)$ & & 0.958 & $-0.11(-1.03-0.92)$ & & 0.836 \\
\hline NL0232A & $0.10(-0.26-0.47)$ & & 0.517 & $0.15(-0.84-1.13)$ & & 0.730 \\
\hline NL0250A & $0.32(0.09-0.60)$ & $* *$ & 0.006 & $0.76(0.21-1.42)$ & $*$ & 0.010 \\
\hline NO01 & $-0.05(-0.28-0.15)$ & & 0.621 & $-0.22(-0.72-0.31)$ & & 0.430 \\
\hline${ }^{\dagger} \mathrm{PL} 03$ & $-0.35(-0.69-0.03)$ & $*$ & 0.043 & $-0.58(-1.24-0.07)$ & + & 0.062 \\
\hline PT0004R & $0.99(0.22-2.01)$ & $* *$ & 0.006 & $2.15(0.47-4.03)$ & $* *$ & 0.004 \\
\hline${ }^{\dagger} \mathrm{PUY}$ & $-0.12(-0.38-0.19)$ & & 0.454 & $-0.16(-0.63-0.41)$ & & 0.568 \\
\hline SI0008R & $-0.09(-0.47-0.25)$ & & 0.680 & $-0.20(-0.86-0.45)$ & & 0.543 \\
\hline${ }^{\dagger} \mathrm{ZUG}$ & $-0.32(-0.52--0.12)$ & $* *$ & 0.002 & $-0.53(-0.82--0.18)$ & $* *$ & 0.002 \\
\hline $\begin{array}{c}\text { European } \\
\text { average }^{\ddagger}\end{array}$ & $0.16 \pm 0.03 \mathrm{ppbv} / \mathrm{yr}$ & & & $0.35 \pm 0.06 \% / \mathrm{yr}$ & & \\
\hline Range $^{\diamond}$ & -1.62 to $1.21 \mathrm{ppbv} / \mathrm{yr}$ & & & -3.12 to $2.16 \% / \mathrm{yr}$ & & \\
\hline
\end{tabular}


Table A5: Quantification of trends (ppbv/yr) in seasonal mean $\mathrm{O}_{3}$ 1996-2005. 95\% confidence interval are given in brackets. Values in bold indicate significant trends $\mathrm{p}$ $<0.1{ }^{\dagger}$ represents high altitude sites $(>1000 \mathrm{~m})$

\begin{tabular}{|c|c|c|c|c|}
\hline ID & $\begin{array}{l}\text { Winter } \\
\text { (DJF) }\end{array}$ & $\begin{array}{l}\text { Spring } \\
\text { (MAM) }\end{array}$ & $\begin{array}{l}\text { Summer } \\
\text { (JJA) }\end{array}$ & $\begin{array}{l}\text { Autumn } \\
\text { (SON) }\end{array}$ \\
\hline AT0002R & $0.25(-0.74-1.35)$ & $0.27(-0.22-1.05)$ & $0.16(-0.38-0.95)$ & $-0.22(-1.10-0.80)$ \\
\hline${ }^{\dagger} \mathrm{AT} 0004 \mathrm{R}$ & $0.27(-0.45-1.21)$ & $0.12(-0.56-0.78)$ & $0.34(-0.30-1.16)$ & $0.08(-0.50-0.75)$ \\
\hline${ }^{\dagger} \mathrm{AT} 0005 \mathrm{R}$ & $0.73(-0.55-1.58)$ & $0.13(-0.24-0.64)$ & $0.04(-0.97-0.83)$ & $0.11(-0.51-0.78)$ \\
\hline${ }^{\dagger} \mathrm{AT} 0034 \mathrm{R}$ & $0.55(-0.36-1.39)$ & $0.32(-0.07-0.81)$ & $0.49(-0.10-1.22)$ & $-0.14(-0.45-0.42)$ \\
\hline AT0044A & $0.35(-0.68-1.31)$ & $-0.18(-0.79-0.50)$ & $0.33(-0.66-1.32)$ & $-0.37(-0.99-0.24)$ \\
\hline AT0052A & $0.46(-1.12-2.18)$ & $0.54(-0.15-1.17)$ & $0.94(-0.18-2.14)$ & $-0.18(-1.06-0.77)$ \\
\hline AT0054A & $0.20(-0.48-1.08)$ & $0.47(-0.11-1.00)$ & $0.28(-0.44-1.02)$ & $-0.13(-0.64-0.61)$ \\
\hline${ }^{\dagger} \mathrm{AT} 0058 \mathrm{~A}$ & $0.28(-0.21-0.71)$ & $-0.05(-0.73-0.59)$ & $0.20(-0.42-1.01)$ & $0.04(-0.52-0.49)$ \\
\hline${ }^{\dagger} \mathrm{AT} 0064 \mathrm{~A}$ & $0.33(-0.17-0.83)$ & $0.35(-0.42-0.92)$ & $0.49(-0.05-1.31)$ & $0.17(-0.44-0.81)$ \\
\hline AT0069A & $0.34(-0.85-1.35)$ & $0.09(-0.62-0.80)$ & $0.14(-0.69-1.06)$ & $-0.29(-1.09-0.41)$ \\
\hline AT0073A & $-0.05(-1.03-0.74)$ & $0.23(-0.38-0.59)$ & $-0.12(-0.96-0.51)$ & $-0.55(-1.22-0.37)$ \\
\hline${ }^{\dagger} \mathrm{AT} 0079 \mathrm{~A}$ & $0.14(-0.29-0.65)$ & $0.26(-0.18-0.82)$ & $0.10(-0.37-0.67)$ & $0.01(-0.47-0.49)$ \\
\hline AT0080A & $0.21(-0.26-0.95)$ & $0.45(-0.26-1.01)$ & $0.14(-0.56-0.80)$ & $-0.06(-0.59-0.50)$ \\
\hline AT0086A & $0.90(0.15-2.07)$ & $0.79(-0.03-1.71)$ & $1.11(0.28-2.26)$ & $0.68(-0.53-1.79)$ \\
\hline${ }^{\dagger} \mathrm{AT} 0089 \mathrm{~A}$ & $0.25(-0.20-0.65)$ & $0.08(-0.29-0.74)$ & $0.37(-0.26-1.23)$ & $0.05(-0.44-0.56)$ \\
\hline AT0094A & $0.39(-0.78-1.51)$ & $0.38(-0.39-1.24)$ & $0.16(-0.75-1.44)$ & $-0.55(-1.45-0.56)$ \\
\hline AT0095A & $0.81(-0.01-2.09)$ & $0.68(0.28-1.23)$ & $0.61(-0.04-1.47)$ & $0.39(-0.43-1.13)$ \\
\hline AT0096A & $0.87(-0.45-2.07)$ & $0.82(0.04-1.55)$ & $0.30(-0.24-1.34)$ & $-0.14(-0.85-0.83)$ \\
\hline AT0101A & $0.52(-0.66-1.77)$ & $0.31(-0.17-0.83)$ & $0.43(-0.20-1.11)$ & $0.17(-0.67-0.91)$ \\
\hline AT0102A & $0.72(-0.29-1.86)$ & $1.01(0.39-1.46)$ & $0.65(0.03-1.80)$ & $-0.02(-0.73-0.87)$ \\
\hline AT0103A & $0.22(-0.97-1.31)$ & $0.17(-0.42-0.76)$ & $0.25(-0.41-0.92)$ & $-0.40(-1.05-0.34)$ \\
\hline AT0105A & $0.78(-0.44-2.11)$ & $1.25(0.64-1.76)$ & $0.78(0.15-1.71)$ & $0.18(-0.78-1.09)$ \\
\hline${ }^{\dagger} \mathrm{AT} 0108 \mathrm{~A}$ & $0.42(-0.43-1.07)$ & $0.72(0.03-1.59)$ & $0.40(-0.42-1.61)$ & $-0.10(-0.82-0.72)$ \\
\hline AT0111A & $0.38(-0.81-1.72)$ & $0.40(0.01-0.98)$ & $0.73(-0.04-1.56)$ & $0.06(-0.84-1.19)$ \\
\hline AT0115A & $0.40(-0.61-1.21)$ & $0.15(-0.33-0.51)$ & $0.01(-0.72-0.98)$ & $-0.12(-0.80-0.51)$ \\
\hline AT0121A & $0.55(-0.36-1.39)$ & $0.32(-0.07-0.81)$ & $0.49(-0.10-1.22)$ & $-0.14(-0.45-0.42)$ \\
\hline AT0122A & $0.43(-0.48-1.30)$ & $0.69(-0.09-1.39)$ & $0.84(0.07-1.87)$ & $-0.23(-1.03-0.42)$ \\
\hline${ }^{\dagger} \mathrm{AT} 0124 \mathrm{~A}$ & $-0.01(-0.52-0.61)$ & $-0.12(-0.51-0.35)$ & $0.18(-0.51-1.11)$ & $-0.04(-0.73-0.58)$ \\
\hline AT0128A & $0.19(-0.85-1.06)$ & $0.03(-0.35-0.73)$ & $0.30(-0.51-1.31)$ & $-0.26(-0.86-0.45)$ \\
\hline AT0134A & $1.30(0.56-1.84)$ & $1.33(0.44-2.25)$ & $0.64(-0.23-1.76)$ & $0.32(-0.30-0.88)$ \\
\hline AT0141A & $0.87(-0.09-1.88)$ & $0.06(-0.56-0.60)$ & $0.30(-0.47-1.08)$ & $-0.02(-0.54-0.55)$ \\
\hline AT0143A & $0.54(-0.12-1.36)$ & $0.27(-0.24-0.89)$ & $0.26(-0.55-1.13)$ & $-0.09(-0.68-0.61)$ \\
\hline${ }^{\dagger} \mathrm{AT} 0146 \mathrm{~A}$ & $0.47(-0.61-1.12)$ & $0.13(-0.32-0.64)$ & $-0.17(-1.20-0.92)$ & $-0.30(-0.70-0.09)$ \\
\hline AT0149A & $0.42(-0.61-1.70)$ & $0.63(0.08-1.30)$ & $0.50(-0.13-1.02)$ & $-0.03(-0.84-0.96)$ \\
\hline AT0153A & $0.27(-1.18-1.50)$ & $0.23(-0.30-0.80)$ & $0.67(-0.37-1.97)$ & $-0.40(-1.20-0.62)$ \\
\hline AT0154A & $0.92(-0.40-1.98)$ & $1.59(0.99-2.69)$ & $0.82(-0.02-1.85)$ & $0.26(-0.67-1.26)$ \\
\hline AT0162A & $0.28(-0.65-1.25)$ & $0.16(-0.31-0.57)$ & $0.21(-0.53-1.03)$ & $-0.33(-0.94-0.47)$ \\
\hline AT0164A & $0.70(0.05-1.93)$ & $0.15(-0.30-0.88)$ & $0.41(-0.24-1.46)$ & $0.13(-0.41-0.78)$ \\
\hline AT0166A & $0.51(-0.07-1.29)$ & $0.49(-0.02-1.29)$ & $0.59(-0.11-1.33)$ & $-0.07(-0.47-0.39)$ \\
\hline AT0167A & $1.31(-0.27-2.86)$ & $1.65(0.85-2.86)$ & $1.15(0.13-2.21)$ & $0.47(-0.81-1.49)$ \\
\hline AT0175A & $0.20(-1.46-1.14)$ & $0.57(0.01-1.24)$ & $0.63(-0.28-1.60)$ & $0.22(-1.27-1.38)$ \\
\hline AT0176A & $0.08(-0.61-1.10)$ & $0.30(-0.30-1.09)$ & $0.50(-0.05-1.20)$ & $-0.04(-0.58-0.66)$ \\
\hline${ }^{\dagger} \mathrm{AT} 0180 \mathrm{~A}$ & $-0.54(-1.30-0.09)$ & $-0.36(-1.15-0.53)$ & $-0.19(-0.82-0.85)$ & $-0.32(-1.09-0.27)$ \\
\hline BE0032R & $-0.19(-1.14-0.91)$ & $0.65(-0.30-1.74)$ & $0.41(-0.26-0.91)$ & $0.03(-1.00-0.66)$ \\
\hline BE0033R & $-0.35(-1.20-0.34)$ & $-0.33(-0.81-0.25)$ & $-0.09(-0.58-0.37)$ & $-0.73(-1.28--0.21)$ \\
\hline BE0035R & $0.17(-0.44-0.77)$ & $0.89(0.05-1.64)$ & $-0.01(-0.55-0.68)$ & $-0.14(-0.78-0.54)$ \\
\hline BE0211A & $0.37(-0.73-1.58)$ & $0.33(-0.65-0.92)$ & $0.21(-0.32-0.92)$ & $-0.45(-1.17-0.24)$ \\
\hline BE0238A & $0.58(-0.38-1.44)$ & $0.54(-0.29-1.26)$ & $0.27(-0.53-0.90)$ & $0.03(-0.79-0.79)$ \\
\hline BE0294A & $0.18(-0.39-1.26)$ & $0.38(-0.76-0.82)$ & $0.25(-0.32-0.88)$ & $-0.68(-1.58-0.23)$ \\
\hline BE0298A & $0.42(-0.61-1.26)$ & $0.28(-0.43-1.00)$ & $0.26(-0.48-0.83)$ & $-0.65(-1.98-0.17)$ \\
\hline BE0302A & $0.28(-0.48-1.22)$ & $0.23(-1.00-1.56)$ & $0.18(-0.33-0.88)$ & $0.05(-0.79-0.56)$ \\
\hline
\end{tabular}


Table A5: continued. Quantification of trends (ppbv/yr) in seasonal mean $\mathrm{O}_{3}$ 19962005. 95\% confidence interval are given in brackets. Values in bold indicate significant trends $\mathrm{p}<0.1 .{ }^{\dagger}$ represents high altitude sites $(>1000 \mathrm{~m})$.

\begin{tabular}{|c|c|c|c|c|}
\hline ID & $\begin{array}{l}\text { Winter } \\
(\mathrm{DJF})\end{array}$ & $\begin{array}{c}\text { Spring } \\
\text { (MAM) } \\
\end{array}$ & $\begin{array}{c}\begin{array}{c}\text { Summer } \\
(\mathrm{JJA})\end{array} \\
\end{array}$ & $\begin{array}{c}\text { Autumn } \\
\text { (SON) }\end{array}$ \\
\hline$\overline{B E 0304 A}$ & $0.16(-0.68-1.15)$ & $0.74(-0.02-1.41)$ & $0.08(-0.61-0.89)$ & $0.28(-0.40-1.07)$ \\
\hline BE0311A & $-0.04(-0.82-0.67)$ & $0.03(-1.00-0.82)$ & $0.04(-0.46-0.73)$ & $0.00(-0.98-0.74)$ \\
\hline BE0345A & $-0.63(-1.37-0.42)$ & $0.28(-0.35-0.92)$ & $0.44(-0.36-1.09)$ & $-0.56(-1.68-0.57)$ \\
\hline${ }^{\dagger} \mathrm{CH} 0001 \mathrm{R}$ & $0.10(-0.20-0.51)$ & $0.20(-0.26-0.66)$ & $0.03(-0.44-0.53)$ & $-0.05(-0.61-0.62)$ \\
\hline CH0002R & $0.80(-0.19-1.84)$ & $0.24(-0.33-0.93)$ & $0.34(-0.21-1.03)$ & $-0.23(-1.07-0.64)$ \\
\hline CH0003R & $0.71(-0.24-1.73)$ & $0.18(-0.36-0.61)$ & $0.14(-0.54-0.79)$ & $-0.37(-0.97-0.54)$ \\
\hline${ }^{\dagger} \mathrm{CH} 0004 \mathrm{R}$ & $0.05(-0.55-0.56)$ & $0.15(-0.42-0.61)$ & $0.07(-0.50-0.93)$ & $-0.31(-0.93-0.45)$ \\
\hline${ }^{\dagger} \mathrm{CH} 0005 \mathrm{R}$ & $0.30(-0.55-1.14)$ & $0.18(-0.26-0.76)$ & $0.26(-0.45-1.03)$ & $0.02(-0.65-0.86)$ \\
\hline CH0019A & $0.04(-1.27-1.07)$ & $0.37(-1.43-1.38)$ & $0.54(-1.16-1.62)$ & $-0.33(-2.21-0.81)$ \\
\hline CH0024A & $0.47(-0.41-1.33)$ & $0.13(-0.70-0.57)$ & $0.04(-0.64-0.69)$ & $-0.21(-1.10-0.66)$ \\
\hline CH0033A & $0.26(-0.45-1.20)$ & $0.06(-0.43-1.43)$ & $0.08(-0.69-1.10)$ & $-0.43(-1.63-0.27)$ \\
\hline${ }^{\dagger} \mathrm{CMN}$ & $-0.08(-0.55-0.49)$ & $0.32(-0.29-1.06)$ & $0.35(-0.21-0.84)$ & $-0.31(-1.19-0.48)$ \\
\hline CZ0001R & $0.00(-0.90-0.78)$ & $-0.33(-0.93-0.27)$ & $-0.63(-1.18-0.09)$ & $-0.20(-0.94-0.57)$ \\
\hline CZ0003R & $0.15(-0.88-1.23)$ & $0.06(-0.45-0.61)$ & $0.05(-0.45-0.68)$ & $-0.01(-0.69-0.80)$ \\
\hline CZ0017A & $-0.10(-0.94-0.84)$ & $0.41(-0.22-0.90)$ & $-0.36(-0.97-0.26)$ & $-0.17(-0.81-0.34)$ \\
\hline CZ0030A & $-0.04(-0.99-0.93)$ & $-0.02(-0.45-0.66)$ & $0.05(-0.68-1.00)$ & $-0.05(-1.20-1.10)$ \\
\hline CZ0041A & $0.08(-1.19-1.27)$ & $0.21(-0.95-1.33)$ & $0.43(-0.29-1.39)$ & $0.09(-0.79-1.14)$ \\
\hline CZ0045A & $0.41(-0.75-1.78)$ & $0.49(0.04-1.08)$ & $0.21(-0.64-1.16)$ & $0.03(-1.11-0.94)$ \\
\hline${ }^{\dagger} \mathrm{CZ} 0049 \mathrm{~A}$ & $0.00(-0.70-0.58)$ & $0.13(-0.42-0.61)$ & $-0.20(-0.92-0.53)$ & $-0.14(-0.73-0.48)$ \\
\hline CZ0051A & $0.64(-0.09-1.43)$ & $0.78(0.05-1.33)$ & $0.15(-0.30-0.81)$ & $0.11(-0.73-0.99)$ \\
\hline${ }^{\dagger} \mathrm{CZ} 0055 \mathrm{~A}$ & $-0.50(-1.59-0.77)$ & $0.15(-1.02-1.33)$ & $0.06(-1.42-2.25)$ & $-0.55(-1.99-0.87)$ \\
\hline CZ0057A & $0.25(-0.72-1.21)$ & $0.25(-0.80-1.14)$ & $-0.35(-1.17-0.54)$ & $-0.34(-1.13-0.25)$ \\
\hline CZ0062A & $0.40(-0.54-1.33)$ & $0.58(-0.26-1.57)$ & $0.62(0.05-1.34)$ & $0.22(-1.05-1.58)$ \\
\hline${ }^{\dagger} \mathrm{DE} 0003 \mathrm{R}$ & $-0.11(-0.63-0.50)$ & $0.59(-0.11-1.08)$ & $0.45(-0.27-1.02)$ & $0.12(-0.41-0.87)$ \\
\hline DE0007R & $0.52(-0.36-1.64)$ & $0.36(-0.15-1.02)$ & $-0.32(-0.81-0.12)$ & $-0.24(-1.02-0.53)$ \\
\hline DE0008R & $0.31(-0.37-0.86)$ & $0.43(-0.30-1.15)$ & $0.04(-0.47-0.92)$ & $0.02(-0.91-1.15)$ \\
\hline DE0009R & $0.28(-0.34-1.05)$ & $0.34(-0.48-1.00)$ & $-0.40(-0.94-0.11)$ & $-0.10(-0.81-0.83)$ \\
\hline DE0035R & $0.40(-0.78-1.53)$ & $0.45(-0.13-1.16)$ & $0.13(-0.62-0.84)$ & $0.09(-1.23-1.28)$ \\
\hline DE0422A & $0.54(-0.35-1.25)$ & $0.29(-0.22-0.94)$ & $0.49(0.02-1.14)$ & $0.00(-0.63-0.69)$ \\
\hline DE0510A & $0.37(-0.49-1.41)$ & $0.06(-0.30-0.64)$ & $0.20(-0.58-0.92)$ & $-0.23(-0.91-0.72)$ \\
\hline DE0514A & $0.40(-0.34-1.31)$ & $0.09(-0.47-0.57)$ & $-0.06(-0.82-0.63)$ & $-0.17(-0.76-0.53)$ \\
\hline DE0556A & $0.61(-0.16-1.48)$ & $0.65(-0.01-1.26)$ & $0.37(-0.09-1.23)$ & $0.14(-0.96-1.38)$ \\
\hline DE0649A & $0.56(-0.36-1.49)$ & $0.38(-0.20-1.09)$ & $0.36(-0.27-1.08)$ & $0.17(-0.52-0.98)$ \\
\hline DE0651A & $0.29(-0.52-1.07)$ & $0.15(-0.44-0.96)$ & $0.28(-0.39-1.08)$ & $-0.03(-1.04-1.01)$ \\
\hline DE0674A & $0.13(-0.51-0.78)$ & $0.15(-0.44-0.72)$ & $0.09(-0.61-0.68)$ & $-0.10(-0.69-0.72)$ \\
\hline DE0679A & $0.31(-0.70-1.21)$ & $0.12(-0.55-0.78)$ & $-0.12(-0.75-0.74)$ & $-0.01(-0.99-0.89)$ \\
\hline DE0680A & $0.47(-0.35-1.20)$ & $0.42(-0.17-1.05)$ & $0.37(-0.29-1.09)$ & $0.13(-0.66-0.91)$ \\
\hline DE0684A & $0.03(-0.77-0.65)$ & $-0.45(-1.13-0.20)$ & $-1.00(-1.87-0.33)$ & $-0.80(-1.60-0.07)$ \\
\hline DE0685A & $0.31(-0.52-1.28)$ & $0.51(-0.28-1.22)$ & $0.26(-0.44-0.93)$ & $0.03(-0.95-0.86)$ \\
\hline DE0686A & $0.32(-0.45-1.40)$ & $0.03(-0.67-0.71)$ & $0.02(-0.54-0.61)$ & $-0.18(-0.98-0.56)$ \\
\hline DE0687A & $0.40(-0.32-1.11)$ & $0.28(-0.31-0.82)$ & $0.09(-0.63-1.03)$ & $-0.07(-0.94-0.74)$ \\
\hline DE0688A & $0.33(-0.49-1.06)$ & $0.17(-0.55-0.76)$ & $-0.04(-0.83-0.79)$ & $-0.13(-0.90-0.69)$ \\
\hline DE0699A & $0.65(-0.49-1.45)$ & $0.75(-0.08-1.47)$ & $0.58(-0.42-1.39)$ & $0.03(-0.99-0.99)$ \\
\hline DE0719A & $0.65(-0.16-1.49)$ & $0.48(-0.30-1.22)$ & $0.48(-0.38-1.29)$ & $0.11(-0.82-1.17)$ \\
\hline DE0732A & $0.28(-0.56-0.97)$ & $0.00(-0.64-0.61)$ & $-0.31(-1.03-0.44)$ & $-0.27(-1.06-0.82)$ \\
\hline DE0735A & $0.48(-0.25-1.19)$ & $0.30(-0.55-1.08)$ & $0.18(-0.62-0.77)$ & $-0.06(-0.66-0.70)$ \\
\hline DE0737A & $0.09(-0.84-1.06)$ & $-0.16(-0.72-0.53)$ & $-0.22(-0.99-0.44)$ & $-0.37(-1.26-0.42)$ \\
\hline DE0738A & $0.43(-0.30-1.16)$ & $-0.08(-0.53-0.54)$ & $0.42(-0.03-0.91)$ & $-0.02(-0.47-0.52)$ \\
\hline DE0739A & $0.35(-0.72-1.10)$ & $0.45(-0.36-1.18)$ & $-0.03(-0.69-0.89)$ & $0.13(-0.80-1.01)$ \\
\hline DE0754A & $0.32(-0.37-1.16)$ & $-0.03(-0.53-0.64)$ & $-0.68(-1.22-0.02)$ & $-0.16(-0.95-0.41)$ \\
\hline DE0844A & $0.33(-0.47-1.13)$ & $0.09(-0.34-0.49)$ & $-0.24(-0.67-0.25)$ & $-0.13(-0.98-0.68)$ \\
\hline
\end{tabular}


Table A5: continued. Quantification of trends (ppbv/yr) in seasonal mean $\mathrm{O}_{3}$ 19962005. 95\% confidence interval are given in brackets. Values in bold indicate significant trends $\mathrm{p}<0.1{ }^{\dagger}$ represents high altitude sites $(>1000 \mathrm{~m})$

\begin{tabular}{|c|c|c|c|c|}
\hline ID & $\begin{array}{l}\text { Winter } \\
\text { (DJF) }\end{array}$ & $\begin{array}{l}\text { Spring } \\
\text { (MAM) }\end{array}$ & $\begin{array}{l}\text { Summer } \\
\text { (JJA) }\end{array}$ & $\begin{array}{c}\text { Autumn } \\
\text { (SON) }\end{array}$ \\
\hline$\overline{\mathrm{DE} 0874 \mathrm{~A}}$ & $0.25(-0.52-1.02)$ & $0.26(-0.27-0.71)$ & $0.07(-0.36-0.58)$ & $0.12(-0.33-0.85)$ \\
\hline DE0907A & $0.32(-0.52-1.14)$ & $0.52(-0.47-1.47)$ & $0.50(-0.19-1.79)$ & $-0.02(-0.65-1.08)$ \\
\hline DE0960A & $0.24(-0.81-1.11)$ & $0.10(-0.72-0.84)$ & $-0.32(-0.91-0.40)$ & $0.06(-0.92-0.75)$ \\
\hline DE0996A & $0.08(-0.45-0.75)$ & $0.02(-0.71-0.63)$ & $0.11(-0.48-0.82)$ & $0.06(-0.62-1.01)$ \\
\hline EE0011R & $-0.41(-0.95-0.31)$ & $-0.41(-0.93-0.08)$ & $-0.36(-1.07-0.34)$ & $-0.49(-1.87-0.33)$ \\
\hline${ }^{\dagger} \mathrm{ES} 0007 \mathrm{R}$ & $-0.65(-1.99-0.26)$ & $-0.85(-2.00-0.26)$ & $-0.90(-1.94-0.84)$ & $-0.79(-2.37-0.55)$ \\
\hline ES1222A & $-0.36(-0.86-0.23)$ & $-0.34(-0.91-0.46)$ & $-0.15(-0.72-0.46)$ & $-1.22(-2.07-0.03)$ \\
\hline ES1400A & $-0.88(-1.66-0.18)$ & $-0.79(-3.15-0.36)$ & $-1.07(-2.08-0.15)$ & $-1.78(-3.07--0.67)$ \\
\hline${ }^{\dagger} \mathrm{ES} 1435 \mathrm{~A}$ & $-0.16(-0.79-0.53)$ & $0.13(-0.52-0.67)$ & $0.84(0.10-1.37)$ & $-0.29(-1.27-0.24)$ \\
\hline${ }^{\dagger} \mathrm{ES} 1437 \mathrm{~A}$ & $-0.72(-1.32--0.17)$ & $-0.18(-0.67-0.28)$ & $-0.05(-1.23-0.86)$ & $-0.89(-1.88-0.00)$ \\
\hline${ }^{\dagger} \mathrm{ES} 1441 \mathrm{~A}$ & $-0.32(-0.95-0.14)$ & $-0.25(-0.71-0.33)$ & $0.10(-0.56-0.52)$ & $-0.63(-1.82-0.19)$ \\
\hline FR08 & $0.97(0.17-1.56)$ & $0.09(-1.12-0.89)$ & $-0.11(-0.98-0.64)$ & $0.46(-0.85-1.36)$ \\
\hline GB0002R & $-0.05(-0.73-0.59)$ & $0.03(-0.30-0.20)$ & $-0.39(-0.82-0.04)$ & $-0.05(-0.45-0.51)$ \\
\hline GB0006R & $0.38(-0.59-1.33)$ & $0.48(0.20-0.75)$ & $-0.07(-0.53-0.54)$ & $0.19(-0.28-0.81)$ \\
\hline GB0013R & $0.31(-0.29-1.21)$ & $0.21(-0.31-0.71)$ & $-0.22(-0.66-0.36)$ & $0.23(-0.11-0.59)$ \\
\hline GB0014R & $0.56(-0.14-1.31)$ & $0.28(-0.46-0.65)$ & $-0.32(-0.65-0.17)$ & $0.16(-0.33-0.69)$ \\
\hline GB0015R & $0.31(-0.37-0.97)$ & $0.24(-0.04-0.70)$ & $-0.17(-0.83-0.54)$ & $-0.02(-0.36-0.84)$ \\
\hline GB0031R & $0.50(-0.43-1.42)$ & $0.46(0.07-0.96)$ & $0.01(-0.49-0.41)$ & $0.79(0.11-1.33)$ \\
\hline GB0033R & $0.28(-0.39-0.93)$ & $0.01(-0.28-0.37)$ & $-0.21(-0.75-0.34)$ & $0.29(0.07-0.59)$ \\
\hline GB0036R & $0.45(-0.49-1.27)$ & $0.39(-0.14-1.09)$ & $-0.18(-0.66-0.54)$ & $0.11(-0.35-0.78)$ \\
\hline GB0037R & $0.42(-0.39-1.15)$ & $0.33(-0.12-0.75)$ & $-0.46(-1.06-0.16)$ & $-0.03(-0.58-0.75)$ \\
\hline GB0038R & $0.42(-0.45-1.14)$ & $0.16(-0.31-0.93)$ & $0.00(-0.63-0.55)$ & $0.28(-0.37-0.77)$ \\
\hline GB0039R & $0.47(-0.34-1.21)$ & $0.29(-0.11-0.88)$ & $-0.22(-0.82-0.44)$ & $0.21(-0.51-1.00)$ \\
\hline GB0044R & $0.00(-0.61-0.83)$ & $0.04(-0.35-0.58)$ & $-0.44(-0.84-0.11)$ & $0.05(-0.34-0.49)$ \\
\hline GB0045R & $0.57(-0.21-1.48)$ & $0.62(0.08-1.48)$ & $0.68(0.02-1.31)$ & $0.60(-0.03-1.41)$ \\
\hline GB0617A & $0.12(-0.55-0.70)$ & $-0.02(-0.67-0.76)$ & $-0.16(-0.75-0.38)$ & $-0.17(-0.88-0.53)$ \\
\hline HPB & $0.09(-0.55-0.82)$ & $0.16(-0.31-0.85)$ & $0.19(-0.47-1.18)$ & $-0.06(-0.88-0.58)$ \\
\hline HU0002R & $-0.27(-1.58-1.02)$ & $-1.88(-2.52--0.81)$ & $-1.27(-2.71-0.08)$ & $-1.60(-2.64--0.42)$ \\
\hline IE31 & $0.27(-0.60-1.06)$ & $0.28(-0.01-0.73)$ & $-0.17(-0.65-0.34)$ & $0.05(-0.30-0.51)$ \\
\hline IT04 & $0.76(-0.13-1.36)$ & $0.52(-0.16-1.43)$ & $0.07(-0.77-0.57)$ & $-0.32(-1.07-0.61)$ \\
\hline LT0015R & $0.42(-0.41-1.74)$ & $0.68(0.15-1.07)$ & $0.22(-0.54-0.62)$ & $0.06(-0.83-0.88)$ \\
\hline LV0010R & $-0.02(-1.03-1.13)$ & $0.16(-0.76-0.71)$ & $-0.67(-1.31--0.18)$ & $-0.65(-1.19-0.10)$ \\
\hline NL0007R & $0.18(-0.46-0.80)$ & $0.47(-0.21-1.03)$ & $-0.02(-0.52-0.55)$ & $-0.14(-0.65-0.42)$ \\
\hline NL0009R & $0.63(-0.48-1.30)$ & $0.45(-0.15-0.97)$ & $0.06(-0.37-0.42)$ & $-0.14(-0.80-0.55)$ \\
\hline NL0010R & $0.19(-0.53-1.02)$ & $0.58(0.08-1.27)$ & $0.16(-0.29-0.64)$ & $-0.24(-0.71-0.41)$ \\
\hline NL0196A & $-0.09(-0.84-0.75)$ & $0.33(-0.27-1.18)$ & $-0.05(-0.62-0.64)$ & $-0.08(-0.66-0.53)$ \\
\hline NL0198A & $0.02(-1.04-1.04)$ & $0.13(-0.54-0.93)$ & $0.16(-0.30-0.68)$ & $-0.03(-0.78-0.88)$ \\
\hline NL0202A & $0.16(-0.71-1.10)$ & $0.36(-0.39-1.11)$ & $0.25(-0.37-0.90)$ & $0.01(-0.72-0.85)$ \\
\hline NL0205A & $-0.15(-0.86-0.73)$ & $0.61(0.03-1.30)$ & $0.37(-0.25-0.86)$ & $-0.22(-0.77-0.72)$ \\
\hline NL0207A & $0.37(-0.66-1.17)$ & $0.29(-0.25-0.91)$ & $0.18(-0.31-0.73)$ & $-0.11(-0.95-0.82)$ \\
\hline NL0209A & $0.20(-0.74-1.05)$ & $0.23(-0.51-1.17)$ & $0.08(-0.45-0.59)$ & $-0.03(-0.54-0.85)$ \\
\hline NL0220A & $0.45(-0.48-1.48)$ & $0.11(-0.44-0.70)$ & $-0.04(-0.49-0.41)$ & $-0.30(-0.70-0.26)$ \\
\hline NL0223A & $0.00(-0.90-0.74)$ & $0.34(-0.50-1.01)$ & $0.20(-0.46-0.82)$ & $0.29(-0.38-1.01)$ \\
\hline NL0226A & $-0.41(-1.14-0.38)$ & $0.30(-0.29-0.93)$ & $0.09(-0.39-0.70)$ & $-0.25(-0.71-0.39)$ \\
\hline NL0227A & $0.45(-0.44-0.97)$ & $0.25(-0.41-0.75)$ & $-0.17(-0.59-0.27)$ & $-0.22(-0.75-0.47)$ \\
\hline NL0228A & $0.17(-0.58-0.97)$ & $0.37(-0.16-0.88)$ & $0.15(-0.34-0.56)$ & $-0.10(-0.54-0.56)$ \\
\hline NL0229A & $0.45(-0.44-1.10)$ & $0.57(-0.07-1.12)$ & $0.67(0.17-1.22)$ & $0.30(-0.31-0.81)$ \\
\hline NL0231A & $0.02(-0.85-0.70)$ & $0.30(-0.55-1.16)$ & $0.08(-0.52-0.47)$ & $-0.12(-0.86-1.00)$ \\
\hline NL0232A & $0.46(-0.38-1.13)$ & $0.19(-0.31-0.78)$ & $-0.31(-0.79-0.34)$ & $-0.14(-0.69-0.43)$ \\
\hline NL0250A & $0.18(-0.51-0.93)$ & $0.19(-0.65-1.00)$ & $0.14(-0.32-0.70)$ & $-0.13(-0.77-0.87)$ \\
\hline NO01 & $0.06(-0.63-0.83)$ & $-0.35(-0.73--0.03)$ & $-0.39(-0.82-0.11)$ & $-0.05(-0.39-0.24)$ \\
\hline
\end{tabular}


Table A5: continued. Quantification of trends (ppbv/yr) in seasonal mean $\mathrm{O}_{3}$ 19962005. 95\% confidence interval are given in brackets. Values in bold indicate significant trends $\mathrm{p}<0.1 .{ }^{\dagger}$ represents high altitude sites $(>1000 \mathrm{~m})$

\begin{tabular}{|c|c|c|c|c|}
\hline ID & $\begin{array}{l}\text { Winter } \\
\text { (DJF) }\end{array}$ & $\begin{array}{l}\text { Spring } \\
\text { (MAM) }\end{array}$ & $\begin{array}{l}\text { Summer } \\
\text { (JJA) }\end{array}$ & $\begin{array}{l}\text { Autumn } \\
\text { (SON) }\end{array}$ \\
\hline${ }^{\dagger}$ PL03 & $-0.32(-0.83-0.17)$ & $-0.59(-1.31-0.23)$ & $-0.32(-1.43-0.69)$ & $-0.19(-1.30-0.97)$ \\
\hline PT0004R & $-0.47(-2.27-1.32)$ & $-0.54(-1.44-2.92)$ & $0.31(-1.67-2.85)$ & $0.20(-1.83-1.53)$ \\
\hline${ }^{\dagger} \mathrm{PUY}$ & $-0.07(-0.48-0.42)$ & $-0.08(-1.10-1.11)$ & $0.24(-0.95-1.13)$ & $0.14(-0.43-0.97)$ \\
\hline SI0008R & $0.18(-0.77-1.84)$ & $-0.51(-1.15-0.30)$ & $-0.42(-1.08-0.35)$ & $-0.59(-0.99-0.01)$ \\
\hline$\dagger \mathrm{ZUG}$ & $-0.40(-0.63--0.04)$ & $-0.14(-0.64-0.38)$ & $-0.25(-0.82-0.20)$ & $-0.46(-0.99--0.04)$ \\
\hline
\end{tabular}


Table A6: Quantification of trends (ppbv/yr) in seasonal $\mathrm{O}_{3} 5^{\text {th }}$ percentiles 1996-2005. 95\% confidence interval are given in brackets. Values in bold indicate significant trends $\mathrm{p}<0.1 .{ }^{\dagger}$ represents high altitude sites $(>1000 \mathrm{~m})$.

\begin{tabular}{|c|c|c|c|c|}
\hline ID & $\begin{array}{l}\text { Winter } \\
\text { (DJF) }\end{array}$ & $\begin{array}{c}\text { Spring } \\
\text { (MAM) } \\
\end{array}$ & $\begin{array}{c}\begin{array}{c}\text { Summer } \\
(\mathrm{JJA})\end{array} \\
\end{array}$ & $\begin{array}{c}\text { Autumn } \\
\text { (SON) }\end{array}$ \\
\hline AT0002R & $0.25(-0.28-1.03)$ & $0.27(-0.50-0.76)$ & $0.16(-0.47-0.56)$ & $-0.22(-0.50-0.62)$ \\
\hline${ }^{\dagger} \mathrm{AT} 0004 \mathrm{R}$ & $0.27(-0.58-1.82)$ & $0.12(-0.76-1.19)$ & $0.34(0.12-1.35)$ & $0.08(-1.10-0.76)$ \\
\hline${ }^{\dagger} \mathrm{AT} 0005 \mathrm{R}$ & $0.73(-0.41-2.13)$ & $0.13(-0.51-0.77)$ & $0.04(-0.50-0.70)$ & $0.11(-0.83-0.58)$ \\
\hline${ }^{\dagger} \mathrm{AT} 0034 \mathrm{R}$ & $0.55(-0.29-1.80)$ & $0.32(-0.15-1.12)$ & $0.49(0.10-1.00)$ & $-0.14(-0.25-0.38)$ \\
\hline AT0044A & $0.35(-0.13-0.37)$ & $-0.18(-0.38-0.54)$ & $0.33(-0.63-0.72)$ & $-0.37(-0.31-0.14)$ \\
\hline AT0052A & $0.46(-0.49-1.23)$ & $0.54(0.00-1.44)$ & $0.94(-0.03-1.55)$ & $-0.18(-0.31-0.50)$ \\
\hline AT0054A & $0.20(-0.48-1.50)$ & $0.47(-0.23-0.87)$ & $0.28(-0.40-0.67)$ & $-0.13(-1.03-0.54)$ \\
\hline${ }^{\dagger} \mathrm{AT} 0058 \mathrm{~A}$ & $0.28(-0.91-0.87)$ & $-0.05(-0.49-0.82)$ & $0.20(-0.25-0.82)$ & $0.04(-0.89-0.46)$ \\
\hline${ }^{\dagger} \mathrm{AT} 0064 \mathrm{~A}$ & $0.33(-0.80-1.08)$ & $0.35(-0.46-1.04)$ & $0.49(0.17-1.17)$ & $0.17(-0.78-1.12)$ \\
\hline AT0069A & $0.34(-0.43-1.63)$ & $0.09(-0.36-1.46)$ & $0.14(-0.46-0.70)$ & $-0.29(-1.12-0.74)$ \\
\hline AT0073A & $-0.05(-0.89-1.44)$ & $0.23(-0.71-1.14)$ & $-0.12(-0.74-0.40)$ & $-0.55(-1.70-0.40)$ \\
\hline${ }^{\dagger} \mathrm{AT} 0079 \mathrm{~A}$ & $0.14(-0.54-0.53)$ & $0.26(-0.50-0.67)$ & $0.10(-0.39-0.67)$ & $0.01(-0.70-0.39)$ \\
\hline AT0080A & $0.21(0.00-0.00)$ & $0.45(0.00-0.33)$ & $0.14(-0.12-0.22)$ & $-0.06(0.00-0.00)$ \\
\hline AT0086A & $0.90(0.00-0.85)$ & $0.79(0.38-1.77)$ & $1.11(0.41-1.50)$ & $0.68(-0.10-0.71)$ \\
\hline${ }^{\dagger} \mathrm{AT} 0089 \mathrm{~A}$ & $0.25(-1.05-0.70)$ & $0.08(-0.48-0.75)$ & $0.37(0.14-1.41)$ & $0.05(-0.98-0.46)$ \\
\hline AT0094A & $0.39(-1.00-1.62)$ & $0.38(-0.83-1.47)$ & $0.16(-0.58-1.14)$ & $-0.55(-1.51-0.67)$ \\
\hline AT0095A & $0.81(-0.20-1.17)$ & $0.68(0.33-1.15)$ & $0.61(0.00-0.99)$ & $0.39(-0.33-0.64)$ \\
\hline AT0096A & $0.87(-0.33-1.99)$ & $0.82(0.51-1.89)$ & $0.30(-0.12-1.15)$ & $-0.14(-0.53-0.94)$ \\
\hline AT0101A & $0.52(0.00-1.81)$ & $0.31(-0.42-0.88)$ & $0.43(0.10-0.86)$ & $0.17(0.00-0.65)$ \\
\hline AT0102A & $0.72(0.00-1.28)$ & $1.01(0.34-1.46)$ & $0.65(0.19-1.34)$ & $-0.02(-0.35-0.46)$ \\
\hline AT0103A & $0.22(-0.50-0.83)$ & $0.17(-0.30-1.16)$ & $0.25(-0.18-0.99)$ & $-0.40(-0.60-0.27)$ \\
\hline AT0105A & $0.78(-0.10-2.35)$ & $1.25(0.63-1.69)$ & $0.78(0.16-1.36)$ & $0.18(-0.42-1.07)$ \\
\hline${ }^{\dagger} \mathrm{AT} 0108 \mathrm{~A}$ & $0.42(-0.08-1.53)$ & $0.72(0.21-1.75)$ & $0.40(-0.15-1.43)$ & $-0.10(-1.15-0.57)$ \\
\hline AT0111A & $0.38(-0.22-0.89)$ & $0.40(-0.17-0.87)$ & $0.73(-0.10-0.89)$ & $0.06(-0.49-0.40)$ \\
\hline AT0115A & $0.40(-0.62-1.88)$ & $0.15(-0.35-1.06)$ & $0.01(-0.57-0.67)$ & $-0.12(-0.75-0.76)$ \\
\hline AT0121A & $0.55(-0.29-1.80)$ & $0.32(-0.15-1.12)$ & $0.49(0.10-1.00)$ & $-0.14(-0.25-0.38)$ \\
\hline AT0122A & $0.43(-0.07-0.04)$ & $0.69(0.16-0.89)$ & $0.84(0.27-0.98)$ & $-0.23(-0.06-0.00)$ \\
\hline${ }^{\dagger}$ AT0124A & $-0.01(-0.58-0.61)$ & $-0.12(-0.65-0.46)$ & $0.18(-0.36-0.82)$ & $-0.04(-0.79-0.40)$ \\
\hline AT0128A & $0.19(-0.10-0.26)$ & $0.03(-0.56-0.40)$ & $0.30(-0.43-0.67)$ & $-0.26(-0.17-0.25)$ \\
\hline AT0134A & $1.30(0.57-2.33)$ & $1.33(0.31-1.76)$ & $0.64(-0.32-1.05)$ & $0.32(-0.32-0.41)$ \\
\hline AT0141A & $0.87(0.00-0.48)$ & $0.06(-0.53-0.38)$ & $0.30(-0.40-0.38)$ & $-0.02(-0.10-0.37)$ \\
\hline AT0143A & $0.54(0.00-0.16)$ & $0.27(-0.12-0.48)$ & $0.26(-0.50-0.24)$ & $-0.09(-0.15-0.00)$ \\
\hline${ }^{\dagger}$ AT0146A & $0.47(-0.69-1.96)$ & $0.13(-0.68-0.92)$ & $-0.17(-1.13-0.41)$ & $-0.30(-0.74-0.06)$ \\
\hline AT0149A & $0.42(-0.19-1.54)$ & $0.63(0.16-1.07)$ & $0.50(-0.14-0.69)$ & $-0.03(-0.59-0.76)$ \\
\hline AT0153A & $0.27(-1.08-1.56)$ & $0.23(-0.11-1.01)$ & $0.67(-0.44-1.35)$ & $-0.40(-0.99-0.65)$ \\
\hline AT0154A & $0.92(-0.68-2.00)$ & $1.59(0.69-2.50)$ & $0.82(0.00-1.61)$ & $0.26(-0.80-1.20)$ \\
\hline AT0162A & $0.28(-0.25-0.20)$ & $0.16(-0.06-0.51)$ & $0.21(-0.17-0.50)$ & $-0.33(-0.19-0.14)$ \\
\hline AT0164A & $0.70(0.05-0.85)$ & $0.15(0.03-0.77)$ & $0.41(0.06-0.57)$ & $0.13(0.16-0.33)$ \\
\hline AT0166A & $0.51(-0.10-0.16)$ & $0.49(0.14-1.22)$ & $0.59(-0.21-0.97)$ & $-0.07(-0.23-0.31)$ \\
\hline AT0167A & $1.31(-0.50-2.95)$ & $1.65(1.02-3.00)$ & $1.15(0.35-2.07)$ & $0.47(-0.84-1.03)$ \\
\hline AT0175A & $0.20(-1.15-1.02)$ & $0.57(-0.16-1.08)$ & $0.63(-0.16-1.15)$ & $0.22(-1.20-1.13)$ \\
\hline AT0176A & $0.08(-0.48-1.57)$ & $0.30(-0.40-1.46)$ & $0.50(-0.06-1.08)$ & $-0.04(-0.98-0.92)$ \\
\hline${ }^{\dagger}$ AT0180A & $-0.54(-2.17-0.00)$ & $-0.36(-1.00-0.31)$ & $-0.19(-0.73-0.50)$ & $-0.32(-1.38-0.47)$ \\
\hline BE0032R & $-0.19(-0.36-0.07)$ & $0.65(-0.23-1.35)$ & $0.41(-0.35-0.87)$ & $0.03(-0.49-0.25)$ \\
\hline BE0033R & $-0.35(-0.16-0.00)$ & $-0.33(-0.75-0.37)$ & $-0.09(-0.67-0.25)$ & $-0.73(-0.32-0.00)$ \\
\hline BE0035R & $0.17(-0.06-0.00)$ & $0.89(0.00-0.49)$ & $-0.01(-0.30-0.21)$ & $-0.14(-0.08-0.00)$ \\
\hline BE0211A & $0.37(-0.12-0.00)$ & $0.33(-0.34-0.39)$ & $0.21(-0.31-0.24)$ & $-0.45(-0.10-0.00)$ \\
\hline BE0238A & $0.58(-0.11-0.80)$ & $0.54(-0.20-1.43)$ & $0.27(-0.10-0.91)$ & $0.03(-0.84-0.76)$ \\
\hline BE0294A & $0.18(-0.06-0.00)$ & $0.38(-0.50-0.50)$ & $0.25(-0.11-0.60)$ & $-0.68(-0.46-0.00)$ \\
\hline BE0298A & $0.42(-0.07-0.00)$ & $0.28(-0.42-0.42)$ & $0.26(-0.33-0.40)$ & $-0.65(-0.12-0.00)$ \\
\hline BE0302A & $0.28(-0.12-0.32)$ & $0.23(-0.62-1.30)$ & $0.18(-0.48-0.69)$ & $0.05(-0.39-0.38)$ \\
\hline
\end{tabular}


Table A6: continued. Quantification of trends (ppbv/yr) in seasonal $\mathrm{O}_{3} 5^{\text {th }}$ percentiles 1996-2005. 95\% confidence interval are given in brackets. Values in bold indicate significant trends $\mathrm{p}<0.1 .{ }^{\dagger}$ represents high altitude sites $(>1000 \mathrm{~m})$.

\begin{tabular}{|c|c|c|c|c|}
\hline ID & $\begin{array}{l}\text { Winter } \\
\text { (DJF) }\end{array}$ & $\begin{array}{c}\text { Spring } \\
(\mathrm{MAM}) \\
\end{array}$ & $\begin{array}{c}\text { Summer } \\
\text { (JJA) }\end{array}$ & $\begin{array}{c}\text { Autumn } \\
(\mathrm{SON})\end{array}$ \\
\hline BE0304A & $0.16(-0.17-0.64)$ & $0.74(-0.07-1.18)$ & $0.08(-0.60-0.56)$ & $0.28(-0.39-0.43)$ \\
\hline BE0311A & $-0.04(-0.93-0.89)$ & $0.03(-0.88-1.00)$ & $0.04(-0.26-0.48)$ & $0.00(-0.75-0.75)$ \\
\hline BE0345A & $-0.63(-0.10-0.00)$ & $0.28(-0.50-0.63)$ & $0.44(-0.24-0.58)$ & $-0.56(-0.07-0.00)$ \\
\hline${ }^{\dagger} \mathrm{CH} 0001 \mathrm{R}$ & $0.10(-0.49-0.49)$ & $0.20(-0.58-0.64)$ & $0.03(-0.62-0.56)$ & $-0.05(-0.69-0.44)$ \\
\hline CH0002R & $0.80(0.03-0.40)$ & $0.24(0.31-1.00)$ & $0.34(0.13-0.94)$ & $-0.23(-0.10-0.16)$ \\
\hline CH0003R & $0.71(0.01-0.47)$ & $0.18(-0.24-0.72)$ & $0.14(-0.28-0.53)$ & $-0.37(-0.16-0.10)$ \\
\hline${ }^{\dagger} \mathrm{CH} 0004 \mathrm{R}$ & $0.05(-0.26-1.55)$ & $0.15(-0.61-0.91)$ & $0.07(-0.29-0.60)$ & $-0.31(-0.99-0.63)$ \\
\hline${ }^{\dagger} \mathrm{CH} 0005 \mathrm{R}$ & $0.30(-0.40-2.09)$ & $0.18(-0.40-1.28)$ & $0.26(0.05-1.31)$ & $0.02(-0.80-1.18)$ \\
\hline CH0019A & $0.04(-0.77-1.18)$ & $0.37(-1.62-1.63)$ & $0.54(-1.10-2.12)$ & $-0.33(-0.85-0.73)$ \\
\hline CH0024A & $0.47(0.04-0.21)$ & $0.13(-0.23-0.27)$ & $0.04(0.01-0.37)$ & $-0.21(-0.03-0.11)$ \\
\hline CH0033A & $0.26(0.03-0.10)$ & $0.06(-0.07-0.72)$ & $0.08(-0.31-0.68)$ & $-0.43(-0.03-0.07)$ \\
\hline${ }^{\dagger} \mathrm{CMN}$ & $-0.08(-0.83-0.20)$ & $0.32(-0.17-1.08)$ & $0.35(-0.11-0.59)$ & $-0.31(-1.36-0.28)$ \\
\hline CZ0001R & $0.00(-0.64-1.30)$ & $-0.33(-0.50-0.54)$ & $-0.63(-1.00-0.00)$ & $-0.20(-1.00-0.50)$ \\
\hline CZ0003R & $0.15(-0.58-1.65)$ & $0.06(-0.40-0.50)$ & $0.05(-0.40-0.44)$ & $-0.01(-0.48-0.76)$ \\
\hline CZ0017A & $-0.10(-1.00-1.07)$ & $0.41(-0.49-1.22)$ & $-0.36(-0.73-0.37)$ & $-0.17(-0.91-0.55)$ \\
\hline CZ0030A & $-0.04(-1.18-1.20)$ & $-0.02(-1.10-0.00)$ & $0.05(-1.01-0.28)$ & $-0.05(-1.29-0.51)$ \\
\hline CZ0041A & $0.08(-0.83-0.95)$ & $0.21(-0.96-0.86)$ & $0.43(-0.46-0.78)$ & $0.09(-0.86-0.51)$ \\
\hline CZ0045A & $0.41(-0.38-2.14)$ & $0.49(-0.30-0.97)$ & $0.21(-0.63-0.63)$ & $0.03(-0.83-0.98)$ \\
\hline${ }^{\dagger} \mathrm{CZ} 0049 \mathrm{~A}$ & $0.00(-0.27-1.53)$ & $0.13(-0.41-0.84)$ & $-0.20(-0.57-0.00)$ & $-0.14(-1.00-0.66)$ \\
\hline CZ0051A & $0.64(-0.17-1.70)$ & $0.78(0.24-1.41)$ & $0.15(-0.12-0.78)$ & $0.11(-0.59-1.20)$ \\
\hline${ }^{\dagger} \mathrm{CZ} 0055 \mathrm{~A}$ & $-0.50(-1.86-0.37)$ & $0.15(-0.70-1.04)$ & $0.06(-1.11-1.82)$ & $-0.55(-1.95-0.34)$ \\
\hline CZ0057A & $0.25(-0.32-1.68)$ & $0.25(-0.75-0.93)$ & $-0.35(-1.19-0.01)$ & $-0.34(-0.91-0.43)$ \\
\hline CZ0062A & $0.40(-0.46-1.54)$ & $0.58(-0.03-1.34)$ & $0.62(-0.09-1.00)$ & $0.22(-1.29-1.50)$ \\
\hline${ }^{\dagger} \mathrm{DE} 0003 \mathrm{R}$ & $-0.11(-0.96-1.21)$ & $0.59(-0.60-1.03)$ & $0.45(-0.13-0.61)$ & $0.12(-1.01-0.59)$ \\
\hline DE0007R & $0.52(-0.06-0.99)$ & $0.36(-0.56-0.49)$ & $-0.32(-0.82--0.10)$ & $-0.24(-0.37-0.00)$ \\
\hline DE0008R & $0.31(-0.20-1.48)$ & $0.43(-0.22-0.99)$ & $0.04(-0.49-0.40)$ & $0.02(-1.21-1.36)$ \\
\hline DE0009R & $0.28(-0.49-1.25)$ & $0.34(-0.88-0.93)$ & $-0.40(-0.41-0.37)$ & $-0.10(-0.72-0.50)$ \\
\hline DE0035R & $0.40(-0.50-1.72)$ & $0.45(-0.14-0.90)$ & $0.13(-0.59-0.43)$ & $0.09(-1.18-1.10)$ \\
\hline DE0422A & $0.54(0.00-0.00)$ & $0.29(0.00-0.00)$ & $0.49(0.00-0.33)$ & $0.00(0.00-0.00)$ \\
\hline DE0510A & $0.37(0.00-0.00)$ & $0.06(-0.30-0.36)$ & $0.20(-0.28-0.83)$ & $-0.23(0.00-0.00)$ \\
\hline DE0514A & $0.40(0.00-0.00)$ & $0.09(-0.36-0.18)$ & $-0.06(-0.44-0.23)$ & $-0.17(0.00-0.00)$ \\
\hline DE0556A & $0.61(-0.17-1.42)$ & $0.65(-0.23-1.20)$ & $0.37(-0.08-0.99)$ & $0.14(-0.90-1.11)$ \\
\hline DE0649A & $0.56(0.00-0.43)$ & $0.38(-0.20-0.75)$ & $0.36(0.09-0.87)$ & $0.17(0.00-0.15)$ \\
\hline DE0651A & $0.29(-0.11-1.13)$ & $0.15(-0.36-0.89)$ & $0.28(-0.49-0.31)$ & $-0.03(-0.66-0.80)$ \\
\hline DE0674A & $0.13(-0.11-0.94)$ & $0.15(-0.19-1.00)$ & $0.09(-0.21-0.66)$ & $-0.10(-0.47-0.62)$ \\
\hline DE0679A & $0.31(-0.25-1.72)$ & $0.12(-0.68-0.54)$ & $-0.12(-0.55-0.50)$ & $-0.01(-0.81-0.85)$ \\
\hline DE0680A & $0.47(0.00-0.58)$ & $0.42(-0.17-1.22)$ & $0.37(0.08-0.75)$ & $0.13(-0.31-0.78)$ \\
\hline DE0684A & $0.03(-0.80-1.46)$ & $-0.45(-1.21-0.65)$ & $-1.00(-1.38-0.08)$ & $-0.80(-1.70-0.09)$ \\
\hline DE0685A & $0.31(0.00-0.91)$ & $0.51(-0.37-1.07)$ & $0.26(-0.20-0.78)$ & $0.03(-0.63-0.67)$ \\
\hline DE0686A & $0.32(-0.36-1.08)$ & $0.03(-0.43-0.76)$ & $0.02(-0.41-0.26)$ & $-0.18(-1.13-0.82)$ \\
\hline DE0687A & $0.40(-0.38-1.19)$ & $0.28(-0.30-0.85)$ & $0.09(-0.25-0.51)$ & $-0.07(-0.71-1.02)$ \\
\hline DE0688A & $0.33(0.00-0.78)$ & $0.17(-0.44-0.87)$ & $-0.04(-0.21-0.66)$ & $-0.13(-0.22-0.73)$ \\
\hline DE0699A & $0.65(-0.14-1.27)$ & $0.75(-0.09-1.42)$ & $0.58(-0.23-0.88)$ & $0.03(-0.51-0.75)$ \\
\hline DE0719A & $0.65(0.00-0.88)$ & $0.48(-0.28-1.29)$ & $0.48(0.00-1.03)$ & $0.11(-0.49-0.91)$ \\
\hline DE0732A & $0.28(-0.17-1.00)$ & $0.00(-1.00-0.25)$ & $-0.31(-0.82-0.00)$ & $-0.27(-1.00-0.61)$ \\
\hline DE0735A & $0.48(-0.18-0.86)$ & $0.30(-0.32-1.20)$ & $0.18(-0.25-0.53)$ & $-0.06(-0.50-1.00)$ \\
\hline DE0737A & $0.09(-0.08-1.44)$ & $-0.16(-0.85-0.69)$ & $-0.22(-0.51-0.37)$ & $-0.37(-1.15-0.98)$ \\
\hline DE0738A & $0.43(0.00-0.38)$ & $-0.08(-0.22-0.22)$ & $0.42(0.06-0.32)$ & $-0.02(0.00-0.00)$ \\
\hline DE0739A & $0.35(0.00-0.93)$ & $0.45(-0.41-1.28)$ & $-0.03(-0.28-0.58)$ & $0.13(-0.37-0.90)$ \\
\hline DE0754A & $0.32(0.00-0.00)$ & $-0.03(-0.10-0.45)$ & $-0.68(-0.36-0.08)$ & $-0.16(-0.08-0.00)$ \\
\hline DE0844A & $0.33(0.00-0.22)$ & $0.09(-0.65-0.55)$ & $-0.24(-0.28-0.30)$ & $-0.13(-0.60-0.26)$ \\
\hline
\end{tabular}


Table A6: continued. Quantification of trends (ppbv/yr) in seasonal $\mathrm{O}_{3} 5^{\text {th }}$ percentiles 1996-2005. 95\% confidence interval are given in brackets. Values in bold indicate significant trends $\mathrm{p}<0.1 .{ }^{\dagger}$ represents high altitude sites $(>1000 \mathrm{~m})$.

\begin{tabular}{|c|c|c|c|c|}
\hline ID & $\begin{array}{l}\text { Winter } \\
\text { (DJF) }\end{array}$ & $\begin{array}{c}\text { Spring } \\
(\mathrm{MAM}) \\
\end{array}$ & $\begin{array}{c}\text { Summer } \\
(\mathrm{JJA})\end{array}$ & $\begin{array}{c}\text { Autumn } \\
(\mathrm{SON})\end{array}$ \\
\hline DE0874A & $0.25(0.00-0.00)$ & $0.26(0.00-0.22)$ & $0.07(0.00-0.33)$ & $0.12(0.00-0.00)$ \\
\hline DE0907A & $0.32(0.00-0.00)$ & $0.52(-0.41-1.16)$ & $0.50(0.00-1.20)$ & $-0.02(0.00-0.00)$ \\
\hline DE0960A & $0.24(-0.34-1.10)$ & $0.10(-0.70-0.52)$ & $-0.32(-0.63-0.26)^{\prime}$ & $0.06(-0.78-0.30)$ \\
\hline DE0996A & $0.08(-0.30-1.26)$ & $0.02(-0.35-0.97)$ & $0.11(-0.22-0.46)$ & $0.06(-0.81-1.17)$ \\
\hline EE0011R & $-0.41(-0.89-0.95)$ & $-0.41(-1.24-0.18)$ & $-0.36(-1.01-0.52)$ & $-0.49(-1.87-0.77)$ \\
\hline${ }^{\dagger} \mathrm{ES} 0007 \mathrm{R}$ & $-0.88(-2.08-0.25)$ & $-0.84(-2.00-0.37)$ & $-0.76(-2.29-0.79)$ & $-0.83(-1.93-0.41)$ \\
\hline ES1222A & $-0.12(-0.23-0.00)$ & $-0.25(-0.58-0.21)$ & $0.01(-0.25-0.42)$ & $-0.33(-0.65-0.00)$ \\
\hline ES1400A & $-1.01(-1.31--0.50)$ & $-0.59(-2.18-0.72)$ & $-0.58(-1.65-0.32)$ & $-1.04(-1.77--0.17)$ \\
\hline${ }^{\dagger} \mathrm{ES} 1435 \mathrm{~A}$ & $-0.54(-0.99-0.11)$ & $0.10(-0.77-0.42)$ & $0.60(0.13-1.03)$ & $-0.10(-0.57-0.29)$ \\
\hline${ }^{\dagger} \mathrm{ES} 1437 \mathrm{~A}$ & $-0.84(-1.48--0.17)$ & $-0.36(-1.05-0.23)$ & $0.00(-0.94-0.93)$ & $-0.67(-1.21--0.14)$ \\
\hline${ }^{\dagger} \mathrm{ES} 1441 \mathrm{~A}$ & $-0.49(-1.08-0.28)$ & $-0.12(-1.13-0.48)$ & $0.34(-0.19-0.83)$ & $-0.56(-1.30-0.24)$ \\
\hline FR08 & $0.97(0.11-1.57)$ & $0.09(-1.15-0.99)$ & $-0.11(-0.75-0.66)$ & $0.46(-1.03-1.23)$ \\
\hline GB0002R & $-0.05(-0.52-0.67)$ & $0.03(-0.26-0.25)$ & $-0.39(-0.40-0.16)$ & $-0.05(-0.16-0.46)$ \\
\hline GB0006R & $0.38(-0.34-1.00)$ & $0.48(0.00-0.72)$ & $-0.07(-0.14-0.47)$ & $0.19(0.00-0.49)$ \\
\hline GB0013R & $0.31(-0.22-1.37)$ & $0.21(-0.49-0.87)$ & $-0.22(-0.76-0.14)$ & $0.23(-0.21-0.67)$ \\
\hline GB0014R & $0.56(0.00-1.00)$ & $0.28(0.00-0.81)$ & $-0.32(-0.20-0.49)$ & $0.16(-0.42-0.88)$ \\
\hline GB0015R & $0.31(-0.68-1.41)$ & $0.24(-0.51-0.88)$ & $-0.17(-0.76-0.38)$ & $-0.02(-0.50-0.63)$ \\
\hline GB0031R & $0.50(-0.63-1.72)$ & $0.46(0.00-1.71)$ & $0.01(-0.72-0.58)$ & $0.79(-0.24-1.28)$ \\
\hline GB0033R & $0.28(-0.59-0.80)$ & $0.01(-0.62-0.57)$ & $-0.21(-0.50-0.38)$ & $0.29(0.00-0.86)$ \\
\hline GB0036R & $0.45(0.00-0.58)$ & $0.39(-0.34-1.35)$ & $-0.18(0.00-0.94)$ & $0.11(-0.50-0.50)$ \\
\hline GB0037R & $0.42(0.00-1.10)$ & $0.33(-0.19-1.00)$ & $-0.46(-0.58-0.00)$ & $-0.03(-0.32-0.78)$ \\
\hline GB0038R & $0.42(-0.14-0.92)$ & $0.16(-0.17-1.20)$ & $0.00(-0.62-0.34)$ & $0.28(-0.57-0.64)$ \\
\hline GB0039R & $0.47(0.00-0.72)$ & $0.29(-0.33-0.93)$ & $-0.22(-0.33-0.62)$ & $0.21(-0.29-0.75)$ \\
\hline GB0044R & $0.00(-0.33-1.00)$ & $0.04(-0.33-0.93)$ & $-0.44(-0.40-0.32)$ & $0.05(-0.61-0.50)$ \\
\hline GB0045R & $0.57(0.00-0.49)$ & $0.62(-0.40-0.98)$ & $0.68(0.00-0.86)$ & $0.60(0.00-0.61)$ \\
\hline GB0617A & $0.12(0.00-0.32)$ & $-0.02(-0.50-0.49)$ & $-0.16(-0.29-0.67)$ & $-0.17(-0.25-0.00)$ \\
\hline HPB & $0.09(-0.47-1.60)$ & $0.16(-0.78-1.35)$ & $0.19(-0.18-0.78)$ & $-0.06(-1.13-0.57)$ \\
\hline HU0002R & $-0.27(-1.04-1.08)$ & $-1.88(-1.86--0.64)$ & $-1.27(-2.13--0.16)$ & $-1.60(-1.33--0.01)$ \\
\hline IE31 & $0.27(-0.58-1.83)$ & $0.28(-0.15-1.13)$ & $-0.17(-1.00-0.50)$ & $0.05(-0.78-0.63)$ \\
\hline IT04 & $0.76(0.05-0.32)$ & $0.52(0.73-1.24)$ & $0.07(0.38-0.91)$ & $-0.32(0.04-0.45)$ \\
\hline LT0015R & $0.42(-0.26-2.06)$ & $0.68(-0.19-1.50)$ & $0.22(-0.50-0.76)$ & $0.06(-1.11-0.15)$ \\
\hline LV0010R & $-0.02(-1.00-1.25)$ & $0.16(-1.30-0.25)$ & $-0.67(-1.27--0.21)$ & $-0.65(-0.93--0.36)$ \\
\hline NL0007R & $0.18(-0.04-0.02)$ & $0.47(-0.03-0.77)$ & $-0.02(-0.40-0.24)$ & $-0.14(-0.11-0.00)$ \\
\hline NL0009R & $0.63(-0.05-0.33)$ & $0.45(-0.35-0.81)$ & $0.06(-0.15-0.36)$ & $-0.14(-0.45-0.23)$ \\
\hline NL0010R & $0.19(-0.07-0.01)$ & $0.58(-0.12-0.44)$ & $0.16(-0.18-0.42)$ & $-0.24(-0.05--0.01)$ \\
\hline NL0196A & $-0.09(-0.06-0.04)$ & $0.33(-0.27-0.60)$ & $-0.05(-0.82-0.27)$ & $-0.08(-0.08--0.03)$ \\
\hline NL0198A & $0.02(-0.08-0.06)$ & $0.13(-0.29-0.87)$ & $0.16(-0.60-0.33)$ & $-0.03(-0.21-0.12)$ \\
\hline NL0202A & $0.16(-0.08-0.08)$ & $0.36(-0.21-0.44)$ & $0.25(-0.41-0.38)$ & $0.01(-0.09-0.04)$ \\
\hline NL0205A & $-0.15(-0.16-0.02)$ & $0.61(-0.07-0.59)$ & $0.37(-0.47-0.13)$ & $-0.22(-0.11-0.02)$ \\
\hline NL0207A & $0.37(-0.05-0.20)$ & $0.29(0.01-1.18)$ & $0.18(-0.20-0.67)$ & $-0.11(-0.57-0.32)$ \\
\hline NL0209A & $0.20(-0.05-0.09)$ & $0.23(-0.05-0.35)$ & $0.08(-0.39-0.24)$ & $-0.03(-0.10-0.06)$ \\
\hline NL0220A & $0.45(-0.08-0.06)$ & $0.11(-0.24-0.60)$ & $-0.04(-0.36-0.35)$ & $-0.30(-0.12-0.02)$ \\
\hline NL0223A & $0.00(-0.06-0.17)$ & $0.34(-0.10-0.47)$ & $0.20(-0.16-0.39)$ & $0.29(0.00-0.07)$ \\
\hline NL0226A & $-0.41(-0.11-0.00)$ & $0.30(-0.06-0.45)$ & $0.09(-0.51-0.30)$ & $-0.25(-0.08--0.01)$ \\
\hline NL0227A & $0.45(-0.10-0.05)$ & $0.25(-0.23-1.12)$ & $-0.17(-0.38-0.39)$ & $-0.22(-0.19--0.02)$ \\
\hline NL0228A & $0.17(-0.08-0.01)$ & $0.37(-0.01-0.85)$ & $0.15(-0.34-0.32)$ & $-0.10(-0.08-0.06)$ \\
\hline NL0229A & $0.45(-0.04-0.03)$ & $0.57(-0.02-0.61)$ & $0.67(0.07-0.53)$ & $0.30(-0.03-0.03)$ \\
\hline NL0231A & $0.02(-0.09-0.06)$ & $0.30(-0.08-1.28)$ & $0.08(-0.31-0.40)$ & $-0.12(-0.20-0.10)$ \\
\hline NL0232A & $0.46(-0.06-0.02)$ & $0.19(-0.20-0.39)$ & $-0.31(-0.31-0.09)$ & $-0.14(-0.04-0.00)$ \\
\hline NL0250A & $0.18(-0.07-0.01)$ & $0.19(-0.28-0.50)$ & $0.14(-0.70-0.26)$ & $-0.13(-0.03-0.03)$ \\
\hline NO01 & $0.06(-1.07-0.52)$ & $-0.35(-0.72-0.25)$ & $-0.39(-0.76-0.00)$ & $-0.05(-0.41-0.00)$ \\
\hline
\end{tabular}


Table A6: continued. Quantification of trends (ppbv/yr) in seasonal $\mathrm{O}_{3} 5^{\text {th }}$ percentiles 1996-2005. 95\% confidence interval are given in brackets. Values in bold indicate significant trends $\mathrm{p}<0.1{ }^{\dagger}$ represents high altitude sites $(>1000 \mathrm{~m})$.

\begin{tabular}{|c|c|c|c|c|}
\hline ID & $\begin{array}{l}\text { Winter } \\
\text { (DJF) }\end{array}$ & $\begin{array}{l}\text { Spring } \\
\text { (MAM) }\end{array}$ & $\begin{array}{l}\text { Summer } \\
\text { (JJA) }\end{array}$ & $\begin{array}{l}\text { Autumn } \\
\text { (SON) }\end{array}$ \\
\hline${ }^{\dagger} \mathrm{PL03}$ & $-0.32(-1.91-0.38)$ & $-0.59(-3.14-0.06)$ & $-0.32(-1.82-1.00)$ & $-0.19(-2.36-0.41)$ \\
\hline PT0004R & $-0.47(-1.21-1.05)$ & $-0.54(-2.17-2.12)$ & $0.31(-2.17-1.15)$ & $0.20(-2.48-0.09)$ \\
\hline${ }^{\dagger} \mathrm{PUY}$ & $-0.07(-0.59-0.45)$ & $-0.08(-0.93-0.93)$ & $0.24(-0.57-2.63)$ & $0.14(-0.89-0.78)$ \\
\hline SI0008R & $0.18(-0.30-0.96)$ & $-0.51(-0.56-0.21)$ & $-0.42(-0.13--0.03)$ & $-0.59(-0.15-0.09)$ \\
\hline$\dagger \mathrm{ZUG}$ & $-0.40(-1.08--0.03)$ & $-0.14(-1.03-0.35)$ & $-0.25(-0.55-0.28)$ & $-0.46(-1.29-0.11)$ \\
\hline
\end{tabular}


Table A7: Quantification of trends (ppbv/yr) in seasonal $\mathrm{O}_{3} 95^{\text {th }}$ percentiles 1996-2005. 95\% confidence interval are given in brackets. Values in bold indicate significant trends $\mathrm{p}<0.1 .{ }^{\dagger}$ represents high altitude sites $(>1000 \mathrm{~m})$.

\begin{tabular}{|c|c|c|c|c|}
\hline ID & $\begin{array}{l}\text { Winter } \\
\text { (DJF) }\end{array}$ & $\begin{array}{l}\text { Spring } \\
\text { (MAM) }\end{array}$ & $\begin{array}{c}\text { Summer } \\
\text { (JJA) }\end{array}$ & $\begin{array}{l}\text { Autumn } \\
\text { (SON) }\end{array}$ \\
\hline AT0002R & $0.25(-0.96-1.05)$ & $0.27(-0.21-1.53)$ & $0.16(-0.45-1.26)$ & $-0.22(-1.24-1.27)$ \\
\hline${ }^{\dagger} \mathrm{AT} 0004 \mathrm{R}$ & $0.27(-0.34-0.83)$ & $0.12(-0.64-1.05)$ & $0.34(-0.30-1.64)$ & $0.08(-0.59-0.86)$ \\
\hline${ }^{\dagger} \mathrm{AT} 0005 \mathrm{R}$ & $0.73(-0.65-1.28)$ & $0.13(-0.44-1.37)$ & $0.04(-1.60-1.27)$ & $0.11(-0.78-1.43)$ \\
\hline${ }^{\dagger} \mathrm{AT} 0034 \mathrm{R}$ & $0.55(-0.31-1.20)$ & $0.32(-0.16-0.94)$ & $0.49(-0.24-1.93)$ & $-0.14(-0.89-0.74)$ \\
\hline AT0044A & $0.35(-0.74-1.20)$ & $-0.18(-1.23-0.97)$ & $0.33(-0.69-2.20)$ & $-0.37(-1.25-0.51)$ \\
\hline AT0052A & $0.46(-1.50-1.97)$ & $0.54(-0.43-1.36)$ & $0.94(-0.40-2.42)$ & $-0.18(-1.37-1.15)$ \\
\hline AT0054A & $0.20(-0.69-0.83)$ & $0.47(-0.28-1.43)$ & $0.28(-0.43-1.32)$ & $-0.13(-0.94-0.78)$ \\
\hline${ }^{\dagger}$ AT0058A & $0.28(-0.08-0.90)$ & $-0.05(-0.78-0.60)$ & $0.20(-0.57-1.19)$ & $0.04(-0.50-0.64)$ \\
\hline${ }^{\dagger} \mathrm{AT} 0064 \mathrm{~A}$ & $0.33(-0.04-0.88)$ & $0.35(-0.99-0.78)$ & $0.49(-0.11-1.81)$ & $0.17(-0.50-0.86)$ \\
\hline AT0069A & $0.34(-0.90-0.96)$ & $0.09(-1.00-1.52)$ & $0.14(-0.85-1.69)$ & $-0.29(-1.03-0.99)$ \\
\hline AT0073A & $-0.05(-1.15-0.24)$ & $0.23(-0.16-0.98)$ & $-0.12(-1.06-1.05)$ & $-0.55(-1.47-0.51)$ \\
\hline${ }^{\dagger} \mathrm{AT} 0079 \mathrm{~A}$ & $0.14(-0.28-0.46)$ & $0.26(-0.50-0.97)$ & $0.10(-0.36-0.87)$ & $0.01(-0.65-0.77)$ \\
\hline AT0080A & $0.21(-0.96-1.02)$ & $0.45(-0.94-1.08)$ & $0.14(-0.44-1.45)$ & $-0.06(-1.44-1.33)$ \\
\hline AT0086A & $0.90(-0.09-2.09)$ & $0.79(-0.84-2.55)$ & $1.11(0.55-2.76)$ & $0.68(-1.45-2.70)$ \\
\hline${ }^{\dagger}$ AT0089A & $0.25(0.00-0.69)$ & $0.08(-0.91-0.62)$ & $0.37(-0.24-1.61)$ & $0.05(-0.44-0.74)$ \\
\hline AT0094A & $0.39(-1.02-0.85)$ & $0.38(-0.75-1.75)$ & $0.16(-1.02-1.67)$ & $-0.55(-1.38-0.67)$ \\
\hline AT0095A & $0.81(-0.03-2.09)$ & $0.68(-0.09-1.68)$ & $0.61(-0.23-1.73)$ & $0.39(-0.91-1.83)$ \\
\hline AT0096A & $0.87(-0.25-1.75)$ & $0.82(0.05-2.00)$ & $0.30(-0.26-1.82)$ & $-0.14(-1.08-0.75)$ \\
\hline AT0101A & $0.52(-0.59-1.50)$ & $0.31(-1.03-1.05)$ & $0.43(-0.45-1.68)$ & $0.17(-1.17-1.25)$ \\
\hline AT0102A & $0.72(-0.50-1.59)$ & $1.01(0.31-2.30)$ & $0.65(-0.49-1.87)$ & $-0.02(-1.70-1.29)$ \\
\hline AT0103A & $0.22(-1.24-1.07)$ & $0.17(-0.96-0.72)$ & $0.25(-0.52-1.10)$ & $-0.40(-1.51-0.64)$ \\
\hline AT0105A & $0.78(-0.45-1.82)$ & $1.25(0.21-2.20)$ & $0.78(-0.07-2.30)$ & $0.18(-1.25-1.28)$ \\
\hline${ }^{\dagger}$ AT0108A & $0.42(-0.70-0.76)$ & $0.72(-0.33-2.08)$ & $0.40(-0.47-1.68)$ & $-0.10(-0.84-1.03)$ \\
\hline AT0111A & $0.38(-0.67-1.50)$ & $0.40(-0.31-2.08)$ & $0.73(0.04-2.51)$ & $0.06(-0.97-1.74)$ \\
\hline AT0115A & $0.40(-0.75-1.14)$ & $0.15(-0.42-1.00)$ & $0.01(-0.70-1.63)$ & $-0.12(-1.17-0.75)$ \\
\hline AT0121A & $0.55(-0.31-1.20)$ & $0.32(-0.16-0.94)$ & $0.49(-0.24-1.93)$ & $-0.14(-0.89-0.74)$ \\
\hline AT0122A & $0.43(-0.95-1.61)$ & $0.69(-0.13-2.00)$ & $0.84(-0.02-2.62)$ & $-0.23(-1.77-0.83)$ \\
\hline${ }^{\dagger} \mathrm{AT} 0124 \mathrm{~A}$ & $-0.01(-0.48-0.69)$ & $-0.12(-1.06-0.37)$ & $0.18(-0.60-1.33)$ & $-0.04(-0.93-0.80)$ \\
\hline AT0128A & $0.19(-1.30-1.48)$ & $0.03(-0.53-1.38)$ & $0.30(-0.61-2.06)$ & $-0.26(-1.08-1.12)$ \\
\hline AT0134A & $1.30(0.00-1.80)$ & $1.33(0.34-2.70)$ & $0.64(-0.10-2.00)$ & $0.32(-0.51-1.58)$ \\
\hline AT0141A & $0.87(-0.40-1.87)$ & $0.06(-0.44-0.99)$ & $0.30(-0.22-1.48)$ & $-0.02(-1.17-0.88)$ \\
\hline AT0143A & $0.54(-0.21-2.25)$ & $0.27(-0.33-1.34)$ & $0.26(-0.22-1.94)$ & $-0.09(-1.16-1.17)$ \\
\hline${ }^{\dagger} \mathrm{AT} 0146 \mathrm{~A}$ & $0.47(-0.70-1.10)$ & $0.13(-0.33-0.72)$ & $-0.17(-1.14-0.96)$ & $-0.30(-1.05-0.38)$ \\
\hline AT0149A & $0.42(-0.63-1.53)$ & $0.63(-0.17-1.87)$ & $0.50(-0.15-1.59)$ & $-0.03(-1.11-1.39)$ \\
\hline AT0153A & $0.27(-1.53-1.36)$ & $0.23(-0.78-0.87)$ & $0.67(-0.68-2.18)$ & $-0.40(-1.48-0.69)$ \\
\hline AT0154A & $0.92(-0.25-2.21)$ & $1.59(0.99-3.23)$ & $0.82(-0.44-1.85)$ & $0.26(-0.67-1.72)$ \\
\hline AT0162A & $0.28(-0.70-1.78)$ & $0.16(-0.48-0.86)$ & $0.21(-0.52-1.39)$ & $-0.33(-1.18-0.92)$ \\
\hline AT0164A & $0.70(-0.80-1.99)$ & $0.15(-0.43-1.54)$ & $0.41(-0.37-2.09)$ & $0.13(-1.30-0.83)$ \\
\hline AT0166A & $0.51(-0.12-1.83)$ & $0.49(0.11-1.56)$ & $0.59(-0.20-2.14)$ & $-0.07(-0.49-0.92)$ \\
\hline AT0167A & $1.31(-0.68-2.37)$ & $1.65(0.93-3.67)$ & $1.15(0.21-2.63)$ & $0.47(-0.84-2.20)$ \\
\hline AT0175A & $0.20(-2.49-1.00)$ & $0.57(-0.20-1.36)$ & $0.63(-0.32-1.81)$ & $0.22(-1.89-1.57)$ \\
\hline AT0176A & $0.08(-0.77-0.70)$ & $0.30(-0.62-1.55)$ & $0.50(0.09-1.91)$ & $-0.04(-0.83-0.78)$ \\
\hline${ }^{\dagger} \mathrm{AT} 0180 \mathrm{~A}$ & $-0.54(-1.09-0.42)$ & $-0.36(-1.19-0.74)$ & $-0.19(-1.02-1.26)$ & $-0.32(-1.11-0.39)$ \\
\hline BE0032R & $-0.19(-0.73-1.05)$ & $0.65(-0.52-2.45)$ & $0.41(-0.99-1.57)$ & $0.03(-1.21-1.22)$ \\
\hline BE0033R & $-0.35(-0.77-0.60)$ & $-0.33(-1.10-0.20)$ & $-0.09(-1.42-1.64)$ & $-0.73(-1.31-0.01)$ \\
\hline BE0035R & $0.17(-0.39-1.09)$ & $0.89(0.01-2.43)$ & $-0.01(-1.62-1.28)$ & $-0.14(-1.68-0.96)$ \\
\hline BE0211A & $0.37(-0.47-2.23)$ & $0.33(-1.50-1.42)$ & $0.21(-1.00-1.57)$ & $-0.45(-1.68-0.05)$ \\
\hline BE0238A & $0.58(-0.35-1.30)$ & $0.54(-0.75-1.74)$ & $0.27(-0.61-1.53)$ & $0.03(-1.30-0.90)$ \\
\hline BE0294A & $0.18(-0.49-1.38)$ & $0.38(-1.62-1.69)$ & $0.25(-0.77-2.07)$ & $-0.68(-1.86-0.00)$ \\
\hline BE0298A & $0.42(-0.38-1.65)$ & $0.28(-1.31-1.84)$ & $0.26(-1.05-2.28)$ & $-0.65(-2.41-0.48)$ \\
\hline BE0302A & $0.28(-0.41-1.18)$ & $0.23(-1.73-2.39)$ & $0.18(-0.43-1.57)$ & $0.05(-1.20-1.40)$ \\
\hline
\end{tabular}


Table A7: continued. Quantification of trends (ppbv/yr) in seasonal $\mathrm{O}_{3} 95^{\text {th }}$ percentiles 1996-2005. 95\% confidence interval are given in brackets. Values in bold indicate significant trends $\mathrm{p}<0.1 .{ }^{\dagger}$ represents high altitude sites $(>1000 \mathrm{~m})$.

\begin{tabular}{|c|c|c|c|c|}
\hline ID & $\begin{array}{l}\text { Winter } \\
\text { (DJF) }\end{array}$ & $\begin{array}{c}\text { Spring } \\
\text { (MAM) }\end{array}$ & $\begin{array}{l}\text { Summer } \\
\text { (JJA) }\end{array}$ & $\begin{array}{c}\text { Autumn } \\
(\mathrm{SON})\end{array}$ \\
\hline BE0304A & $0.16(-0.54-0.99)$ & $0.74(-0.21-2.17)$ & $0.08(-1.29-1.89)$ & $0.28(-1.06-2.29)$ \\
\hline BE0311A & $-0.04(-0.77-0.59)$ & $0.03(-1.87-1.46)$ & $0.04(-0.71-1.37)$ & $0.00(-1.25-1.15)$ \\
\hline BE0345A & $-0.63(-0.69-0.34)$ & $0.28(-1.59-2.07)$ & $0.44(-1.04-2.97)$ & $-0.56(-2.47-1.49)$ \\
\hline${ }^{\dagger} \mathrm{CH} 0001 \mathrm{R}$ & $0.10(-0.16-0.82)$ & $0.20(-0.53-0.95)$ & $0.03(-0.44-0.62)$ & $-0.05(-0.73-0.58)$ \\
\hline CH0002R & $0.80(-0.47-1.89)$ & $0.24(-0.61-1.10)$ & $0.34(-0.33-1.62)$ & $-0.23(-1.67-0.85)$ \\
\hline CH0003R & $0.71(-0.68-1.07)$ & $0.18(-0.69-1.42)$ & $0.14(-0.97-1.53)$ & $-0.37(-1.53-0.66)$ \\
\hline${ }^{\dagger} \mathrm{CH} 0004 \mathrm{R}$ & $0.05(-0.89-0.15)$ & $0.15(-0.81-1.01)$ & $0.07(-0.28-1.62)$ & $-0.31(-1.53-0.79)$ \\
\hline${ }^{\dagger} \mathrm{CH} 0005 \mathrm{R}$ & $0.30(-0.67-0.53)$ & $0.18(-0.73-0.78)$ & $0.26(-0.49-1.80)$ & $0.02(-1.09-0.61)$ \\
\hline CH0019A & $0.04(-1.95-0.67)$ & $0.37(-2.70-1.22)$ & $0.54(-1.45-2.14)$ & $-0.33(-3.66-0.45)$ \\
\hline CH0024A & $0.47(-0.78-1.35)$ & $0.13(-1.21-1.05)$ & $0.04(-1.41-0.87)$ & $-0.21(-1.65-0.83)$ \\
\hline CH0033A & $0.26(-1.17-1.49)$ & $0.06(-1.80-1.34)$ & $0.08(-1.73-1.63)$ & $-0.43(-2.73-0.63)$ \\
\hline${ }^{\dagger} \mathrm{CMN}$ & $-0.08(-0.75-0.83)$ & $0.32(-0.67-0.98)$ & $0.35(-0.82-1.48)$ & $-0.31(-1.58-0.80)$ \\
\hline CZ0001R & $0.00(-1.13-0.60)$ & $-0.33(-1.43-0.40)$ & $-0.63(-1.09-0.15)$ & $-0.20(-1.16-1.02)$ \\
\hline CZ0003R & $0.15(-1.06-0.97)$ & $0.06(-1.00-0.81)$ & $0.05(-0.60-1.02)$ & $-0.01(-1.23-1.46)$ \\
\hline CZ0017A & $-0.10(-1.50-0.64)$ & $0.41(-0.98-0.91)$ & $-0.36(-1.08-0.45)$ & $-0.17(-1.25-0.68)$ \\
\hline CZ0030A & $-0.04(-1.16-1.00)$ & $-0.02(-0.96-1.04)$ & $0.05(-0.98-1.24)$ & $-0.05(-1.38-1.62)$ \\
\hline CZ0041A & $0.08(-1.35-1.27)$ & $0.21(-1.01-2.25)$ & $0.43(-0.75-2.30)$ & $0.09(-1.46-1.69)$ \\
\hline CZ0045A & $0.41(-0.73-1.46)$ & $0.49(-0.02-1.50)$ & $0.21(-0.85-1.75)$ & $0.03(-1.95-1.52)$ \\
\hline${ }^{\dagger} \mathrm{CZ} 0049 \mathrm{~A}$ & $0.00(-0.88-0.23)$ & $0.13(-0.73-0.87)$ & $-0.20(-1.23-0.79)$ & $-0.14(-1.16-0.48)$ \\
\hline CZ0051A & $0.64(-0.52-1.36)$ & $0.78(-0.48-1.49)$ & $0.15(-0.64-0.70)$ & $0.11(-0.90-1.10)$ \\
\hline${ }^{\dagger} \mathrm{CZ0055A}$ & $-0.50(-1.25-0.88)$ & $0.15(-2.17-2.08)$ & $0.06(-2.02-3.12)$ & $-0.55(-1.96-0.95)$ \\
\hline CZ0057A & $0.25(-0.90-0.96)$ & $0.25(-1.02-1.52)$ & $-0.35(-1.49-1.03)$ & $-0.34(-1.25-0.62)$ \\
\hline CZ0062A & $0.40(-0.74-0.75)$ & $0.58(-0.91-1.80)$ & $0.62(0.31-1.88)$ & $0.22(-1.21-2.83)$ \\
\hline${ }^{\dagger} \mathrm{DE} 0003 \mathrm{R}$ & $-0.11(-0.69-0.34)$ & $0.59(-0.22-1.60)$ & $0.45(-0.12-2.47)$ & $0.12(-0.96-1.09)$ \\
\hline DE0007R & $0.52(-0.20-1.30)$ & $0.36(-0.76-1.62)$ & $-0.32(-0.98-1.03)$ & $-0.24(-0.98-1.48)$ \\
\hline DE0008R & $0.31(-0.69-0.62)$ & $0.43(-1.15-1.46)$ & $0.04(-0.46-1.59)$ & $0.02(-1.12-1.68)$ \\
\hline DE0009R & $0.28(-0.30-0.50)$ & $0.34(-0.63-1.54)$ & $-0.40(-1.40-0.15)$ & $-0.10(-0.50-0.76)$ \\
\hline DE0035R & $0.40(-0.79-1.03)$ & $0.45(-0.75-1.75)$ & $0.13(-0.74-1.59)$ & $0.09(-1.06-2.00)$ \\
\hline DE0422A & $0.54(0.17-1.97)$ & $0.29(-0.80-1.78)$ & $0.49(-0.89-1.95)$ & $0.00(-0.98-1.67)$ \\
\hline DE0510A & $0.37(-0.72-1.27)$ & $0.06(-0.70-1.48)$ & $0.20(-0.80-1.78)$ & $-0.23(-1.67-1.08)$ \\
\hline DE0514A & $0.40(-0.78-1.33)$ & $0.09(-0.77-1.21)$ & $-0.06(-1.02-1.42)$ & $-0.17(-1.00-0.80)$ \\
\hline DE0556A & $0.61(-0.51-0.66)$ & $0.65(-0.54-1.91)$ & $0.37(-0.08-2.02)$ & $0.14(-0.59-1.62)$ \\
\hline DE0649A & $0.56(-0.27-1.42)$ & $0.38(-0.71-1.92)$ & $0.36(-0.39-1.42)$ & $0.17(-0.97-1.29)$ \\
\hline DE0651A & $0.29(-0.74-0.59)$ & $0.15(-1.17-1.84)$ & $0.28(0.14-1.88)$ & $-0.03(-1.13-1.45)$ \\
\hline DE0674A & $0.13(-0.60-0.24)$ & $0.15(-0.63-1.16)$ & $0.09(-1.06-1.29)$ & $-0.10(-0.95-0.50)$ \\
\hline DE0679A & $0.31(-0.91-0.43)$ & $0.12(-0.95-0.87)$ & $-0.12(-0.98-0.78)$ & $-0.01(-1.73-0.90)$ \\
\hline DE0680A & $0.47(-0.17-1.01)$ & $0.42(-0.41-1.87)$ & $0.37(-0.64-1.42)$ & $0.13(-0.74-1.00)$ \\
\hline DE0684A & $0.03(-1.21-0.54)$ & $-0.45(-1.95-0.88)$ & $-1.00(-2.09-1.20)$ & $-0.80(-2.60-0.50)$ \\
\hline DE0685A & $0.31(-0.48-1.50)$ & $0.51(-0.72-1.80)$ & $0.26(-0.48-1.52)$ & $0.03(-1.35-0.91)$ \\
\hline DE0686A & $0.32(-0.49-0.70)$ & $0.03(-1.00-1.13)$ & $0.02(-1.00-1.30)$ & $-0.18(-1.30-0.44)$ \\
\hline DE0687A & $0.40(-0.43-0.52)$ & $0.28(-0.50-1.56)$ & $0.09(-0.97-1.63)$ & $-0.07(-1.18-0.94)$ \\
\hline DE0688A & $0.33(-0.58-0.84)$ & $0.17(-1.04-1.33)$ & $-0.04(-1.16-0.66)$ & $-0.13(-1.44-0.63)$ \\
\hline DE0699A & $0.65(-0.55-1.35)$ & $0.75(-0.50-1.83)$ & $0.58(-0.30-2.17)$ & $0.03(-1.50-1.34)$ \\
\hline DE0719A & $0.65(-0.60-1.08)$ & $0.48(-1.01-1.51)$ & $0.48(-1.11-1.71)$ & $0.11(-1.21-1.50)$ \\
\hline DE0732A & $0.28(-0.59-0.72)$ & $0.00(-0.83-1.57)$ & $-0.31(-1.50-0.94)$ & $-0.27(-1.00-1.03)$ \\
\hline DE0735A & $0.48(-0.45-0.86)$ & $0.30(-0.93-1.68)$ & $0.18(-0.52-1.94)$ & $-0.06(-1.16-0.75)$ \\
\hline DE0737A & $0.09(-1.16-0.36)$ & $-0.16(-1.30-1.07)$ & $-0.22(-1.37-0.80)$ & $-0.37(-1.92-0.29)$ \\
\hline DE0738A & $0.43(-0.68-0.80)$ & $-0.08(-1.25-0.85)$ & $0.42(-0.57-1.44)$ & $-0.02(-1.16-1.44)$ \\
\hline DE0739A & $0.35(-0.90-0.80)$ & $0.45(-0.81-2.28)$ & $-0.03(-1.29-1.24)$ & $0.13(-1.29-1.00)$ \\
\hline DE0754A & $0.32(-0.75-1.20)$ & $-0.03(-1.94-1.00)$ & $-0.68(-1.87-0.28)$ & $-0.16(-1.49-1.40)$ \\
\hline DE0844A & $0.33(-0.50-0.61)$ & $0.09(-0.51-0.83)$ & $-0.24(-1.18-0.51)$ & $-0.13(-1.04-1.13)$ \\
\hline
\end{tabular}


Table A7: continued. Quantification of trends (ppbv/yr) in seasonal $\mathrm{O}_{3} 95^{\text {th }}$ percentiles 1996-2005. 95\% confidence interval are given in brackets. Values in bold indicate significant trends $\mathrm{p}<0.1 .{ }^{\dagger}$ represents high altitude sites $(>1000 \mathrm{~m})$.

\begin{tabular}{|c|c|c|c|c|}
\hline ID & $\begin{array}{l}\text { Winter } \\
\text { (DJF) }\end{array}$ & $\begin{array}{c}\text { Spring } \\
\text { (MAM) } \\
\end{array}$ & $\begin{array}{c}\text { Summer } \\
(\text { JJA })\end{array}$ & $\begin{array}{c}\text { Autumn } \\
(\mathrm{SON})\end{array}$ \\
\hline DE0874A & $0.25(-0.50-1.09)$ & $0.26(-0.39-1.16)$ & $0.07(-0.93-0.84)$ & $0.12(-1.00-1.02)$ \\
\hline DE0907A & $0.32(-0.83-1.53)$ & $0.52(-1.00-2.12)$ & $0.50(-0.72-2.01)$ & $-0.02(-1.47-2.19)$ \\
\hline DE0960A & $0.24(-0.69-0.78)$ & $0.10(-1.26-1.43)$ & $-0.32(-1.50-1.00)$ & $0.06(-0.60-1.24)$ \\
\hline DE0996A & $0.08(-0.70-0.43)$ & $0.02(-1.15-1.32)$ & $0.11(-0.55-1.35)$ & $0.06(-0.87-1.67)$ \\
\hline EE0011R & $-0.41(-1.00--0.02)$ & $-0.41(-1.28-0.77)$ & $-0.36(-1.73-0.85)$ & $-0.49(-2.04-0.32)$ \\
\hline${ }^{\dagger} \mathrm{ES} 0007 \mathrm{R}$ & $-0.70(-1.93-0.11)$ & $-0.89(-2.00-0.37)$ & $-1.09(-2.39-0.75)$ & $-0.85(-2.96-0.93)$ \\
\hline ES1222A & $-0.29(-1.35-0.48)$ & $-0.34(-1.17-0.75)$ & $-0.52(-1.40-0.63)$ & $-2.28(-4.31-0.14)$ \\
\hline ES1400A & $-0.87(-1.69-0.19)$ & $-1.20(-3.60-0.00)$ & $-1.30(-2.79-0.76)$ & $-2.94(-4.38--1.20)$ \\
\hline${ }^{\dagger} \mathrm{ES} 1435 \mathrm{~A}$ & $0.11(-0.62-0.94)$ & $0.34(-0.57-1.23)$ & $1.09(0.26-1.99)$ & $-0.55(-2.26-0.66)$ \\
\hline${ }^{\dagger} \mathrm{ES} 1437 \mathrm{~A}$ & $-0.70(-1.52--0.13)$ & $0.15(-0.64-1.04)$ & $-0.18(-1.88-0.92)$ & $-1.12(-2.75--0.28)$ \\
\hline${ }^{\dagger} \mathrm{ES} 1441 \mathrm{~A}$ & $-0.53(-1.22-0.00)$ & $-0.13(-0.56-0.86)$ & $-0.09(-0.92-0.60)$ & $-0.68(-2.81-0.75)$ \\
\hline FR08 & $0.97(-0.03-2.11)$ & $0.09(-1.03-0.97)$ & $-0.11(-1.09-1.22)$ & $0.46(-1.34-2.42)$ \\
\hline GB0002R & $-0.05(-0.69-0.14)$ & $0.03(-0.57-0.50)$ & $-0.39(-1.74--0.01)$ & $-0.05(-0.90-0.17)$ \\
\hline GB0006R & $0.38(-0.75-1.14)$ & $0.48(0.00-0.83)$ & $-0.07(-1.56-0.00)$ & $0.19(-0.49-0.67)$ \\
\hline GB0013R & $0.31(0.00-0.62)$ & $0.21(-0.50-1.00)$ & $-0.22(-1.85-0.74)$ & $0.23(-0.16-0.71)$ \\
\hline GB0014R & $0.56(0.00-0.96)$ & $0.28(-0.76-1.01)$ & $-0.32(-2.25-0.53)$ & $0.16(-0.34-0.69)$ \\
\hline GB0015R & $0.31(-0.17-0.65)$ & $0.24(0.11-0.78)$ & $-0.17(-1.32-0.86)$ & $-0.02(-0.39-0.75)$ \\
\hline GB0031R & $0.50(0.00-0.85)$ & $0.46(0.00-1.00)$ & $0.01(-1.66-1.40)$ & $0.79(0.00-1.28)$ \\
\hline GB0033R & $0.28(-0.13-0.58)$ & $0.01(-0.45-0.41)$ & $-0.21(-1.69-0.33)$ & $0.29(-0.14-0.53)$ \\
\hline GB0036R & $0.45(0.00-0.96)$ & $0.39(-0.51-1.43)$ & $-0.18(-2.20-0.50)$ & $0.11(-0.52-1.00)$ \\
\hline GB0037R & $0.42(-0.40-1.00)$ & $0.33(-0.45-1.05)$ & $-0.46(-2.21-1.00)$ & $-0.03(-0.87-0.76)$ \\
\hline GB0038R & $0.42(-0.19-0.84)$ & $0.16(-0.77-1.13)$ & $0.00(-2.13-1.27)$ & $0.28(-0.30-1.01)$ \\
\hline GB0039R & $0.47(-0.50-1.23)$ & $0.29(-0.10-1.53)$ & $-0.22(-1.76-1.00)$ & $0.21(-0.33-1.02)$ \\
\hline GB0044R & $0.00(-0.43-0.49)$ & $0.04(-0.76-0.50)$ & $-0.44(-2.61--0.25)$ & $0.05(-0.57-0.56)$ \\
\hline GB0045R & $0.57(0.20-1.65)$ & $0.62(-0.29-2.40)$ & $0.68(-1.04-2.88)$ & $0.60(-0.53-1.75)$ \\
\hline GB0617A & $0.12(-0.45-0.34)$ & $-0.02(-0.86-0.96)$ & $-0.16(-1.69-0.50)$ & $-0.17(-1.00-0.69)$ \\
\hline HPB & $0.09(-0.74-0.32)$ & $0.16(-0.44-1.43)$ & $0.19(-0.68-2.25)$ & $-0.06(-1.10-0.82)$ \\
\hline HU0002R & $-0.27(-1.98-1.49)$ & $-1.88(-4.04--0.99)$ & $-1.27(-2.76-0.60)$ & $-1.60(-3.51--0.26)$ \\
\hline IE31 & $0.27(-0.17-0.42)$ & $0.28(-0.39-0.65)$ & $-0.17(-1.74-0.00)$ & $0.05(-0.28-0.33)$ \\
\hline IT04 & $0.76(-0.55-2.96)$ & $0.52(-1.90-1.00)$ & $0.07(-2.73-0.76)$ & $-0.32(-3.31-0.72)$ \\
\hline LT0015R & $0.42(-0.73-0.88)$ & $0.68(0.00-1.38)$ & $0.22(-0.76-0.98)$ & $0.06(-1.46-1.78)$ \\
\hline LV0010R & $-0.02(-0.95-0.70)$ & $0.16(-1.36-1.61)$ & $-0.67(-1.73--0.40)$ & $-0.65(-1.45-0.21)$ \\
\hline NL0007R & $0.18(-0.48-0.92)$ & $0.47(-0.36-1.73)$ & $-0.02(-1.85-1.30)$ & $-0.14(-0.70-0.73)$ \\
\hline NL0009R & $0.63(-0.10-1.34)$ & $0.45(-0.22-1.16)$ & $0.06(-0.46-1.20)$ & $-0.14(-0.77-0.69)$ \\
\hline NL0010R & $0.19(-0.37-1.31)$ & $0.58(-0.08-1.77)$ & $0.16(-1.06-1.50)$ & $-0.24(-0.86-0.77)$ \\
\hline NL0196A & $-0.09(-0.58-0.66)$ & $0.33(-0.63-1.98)$ & $-0.05(-1.37-1.86)$ & $-0.08(-0.84-0.68)$ \\
\hline NL0198A & $0.02(-0.76-1.02)$ & $0.13(-0.71-0.93)$ & $0.16(-1.20-2.18)$ & $-0.03(-0.79-1.19)$ \\
\hline NL0202A & $0.16(-0.66-0.77)$ & $0.36(-0.80-1.83)$ & $0.25(-1.22-2.32)$ & $0.01(-1.09-0.69)$ \\
\hline NL0205A & $-0.15(-0.52-0.59)$ & $0.61(-0.14-1.74)$ & $0.37(-1.01-2.55)$ & $-0.22(-1.04-1.39)$ \\
\hline NL0207A & $0.37(-0.46-0.77)$ & $0.29(-0.33-1.16)$ & $0.18(-1.00-1.93)$ & $-0.11(-0.95-0.71)$ \\
\hline NL0209A & $0.20(-0.60-0.72)$ & $0.23(-0.96-1.41)$ & $0.08(-1.19-1.88)$ & $-0.03(-0.86-1.36)$ \\
\hline NL0220A & $0.45(-0.70-1.05)$ & $0.11(-0.65-0.86)$ & $-0.04(-1.19-1.30)$ & $-0.30(-0.93-0.39)$ \\
\hline NL0223A & $0.00(-0.80-0.49)$ & $0.34(-1.13-1.41)$ & $0.20(-1.90-1.53)$ & $0.29(-0.62-1.41)$ \\
\hline NL0226A & $-0.41(-0.79-0.54)$ & $0.30(-0.50-1.39)$ & $0.09(-1.19-1.91)$ & $-0.25(-0.81-1.25)$ \\
\hline NL0227A & $0.45(-0.31-0.82)$ & $0.25(-0.49-0.77)$ & $-0.17(-1.17-0.44)$ & $-0.22(-0.71-0.40)$ \\
\hline NL0228A & $0.17(-0.29-1.08)$ & $0.37(-0.16-1.01)$ & $0.15(-0.72-1.50)$ & $-0.10(-0.67-0.81)$ \\
\hline NL0229A & $0.45(-0.35-1.33)$ & $0.57(-0.30-1.45)$ & $0.67(-0.44-2.48)$ & $0.30(-0.23-1.39)$ \\
\hline NL0231A & $0.02(-0.63-0.47)$ & $0.30(-0.90-1.49)$ & $0.08(-1.25-1.77)$ & $-0.12(-1.25-1.35)$ \\
\hline NL0232A & $0.46(-0.31-1.66)$ & $0.19(-0.54-1.11)$ & $-0.31(-1.88-1.44)$ & $-0.14(-1.23-0.50)$ \\
\hline NL0250A & $0.18(-0.27-1.00)$ & $0.19(-0.37-1.25)$ & $0.14(-0.43-2.25)$ & $-0.13(-0.74-0.99)$ \\
\hline NO01 & $0.06(-0.58-0.50)$ & $-0.35(-0.63-0.42)$ & $-0.39(-1.40-0.13)$ & $-0.05(-0.25-0.52)$ \\
\hline
\end{tabular}


Table A7: continued. Quantification of trends (ppbv/yr) in seasonal $\mathrm{O}_{3} 95^{\text {th }}$ percentiles 1996-2005. 95\% confidence interval are given in brackets. Values in bold indicate significant trends $\mathrm{p}<0.1{ }^{\dagger}$ represents high altitude sites $(>1000 \mathrm{~m})$.

\begin{tabular}{|c|c|c|c|c|}
\hline ID & $\begin{array}{l}\text { Winter } \\
\text { (DJF) }\end{array}$ & $\begin{array}{c}\text { Spring } \\
\text { (MAM) }\end{array}$ & $\begin{array}{c}\text { Summer } \\
\text { (JJA) }\end{array}$ & $\begin{array}{c}\text { Autumn } \\
\text { (SON) }\end{array}$ \\
\hline${ }^{\dagger} \mathrm{PLO3}$ & $-0.32(-0.80-0.52)$ & $-0.59(-1.14-0.39)$ & $-0.32(-1.40-0.65)$ & $-0.19(-1.00-1.15)$ \\
\hline PT0004R & $-0.47(-2.23-1.75)$ & $-0.54(-1.27-3.70)$ & $0.31(-0.83-5.37)$ & $0.20(-1.93-2.90)$ \\
\hline${ }^{\dagger} \mathrm{PUY}$ & $-0.07(-0.45-0.70)$ & $-0.08(-0.95-1.82)$ & $0.24(-1.41-1.29)$ & $0.14(-0.79-1.17)$ \\
\hline SI0008R & $0.18(-1.64-1.64)$ & $-0.51(-0.85-1.02)$ & $-0.42(-0.83-1.51)$ & $-0.59(-2.62-0.09)$ \\
\hline${ }^{\dagger} \mathrm{ZUG}$ & $-0.40(-0.59-0.14)$ & $-0.14(-0.83-0.61)$ & $-0.25(-0.99-0.73)$ & $-0.46(-1.19-0.02)$ \\
\hline
\end{tabular}




\section{CHIMERE model trends}

A compilation of loess trends of monthly mean $\mathrm{O}_{3}$ for data extracted from CHIMERE CTM at the locations of all stations in this study are given in Figure ?? in addition to annual $5^{\text {th }}$ and $95^{\text {th }}$ percentiles of $\mathrm{O}_{3} .75 \%$ coverage of monthly data was required to calculate statistics.

Quantification of trends in annual mean, $5^{\text {th }}$ and $95^{\text {th }}$ percentiles for data extracted from CHIMERE CTM at the locations of all stations in this study are given in Tables ?? - ??, respectively. 

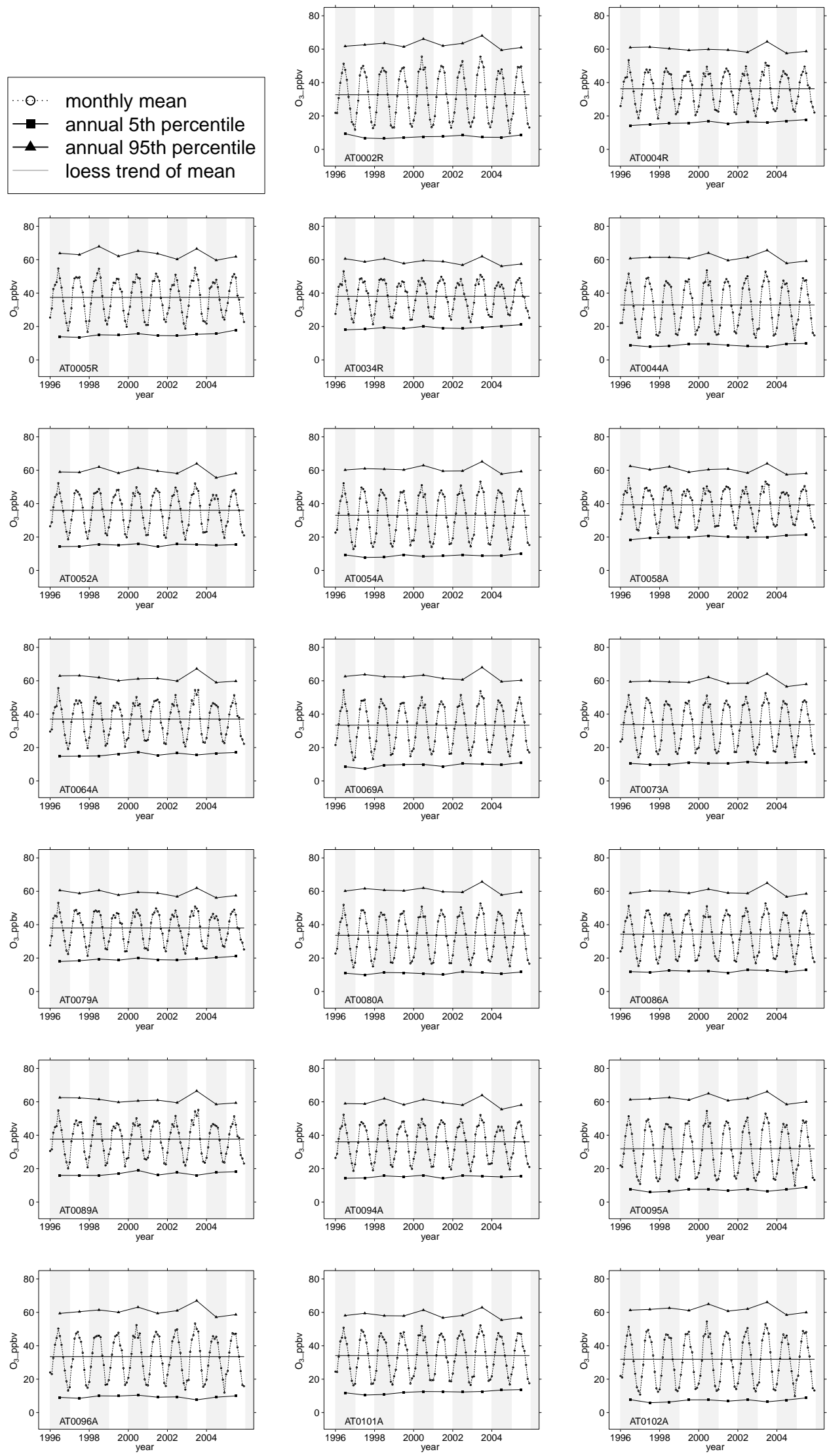

Figure B1: Loess trends of CHIMERE simulated monthly mean $\mathrm{O}_{3}$ 

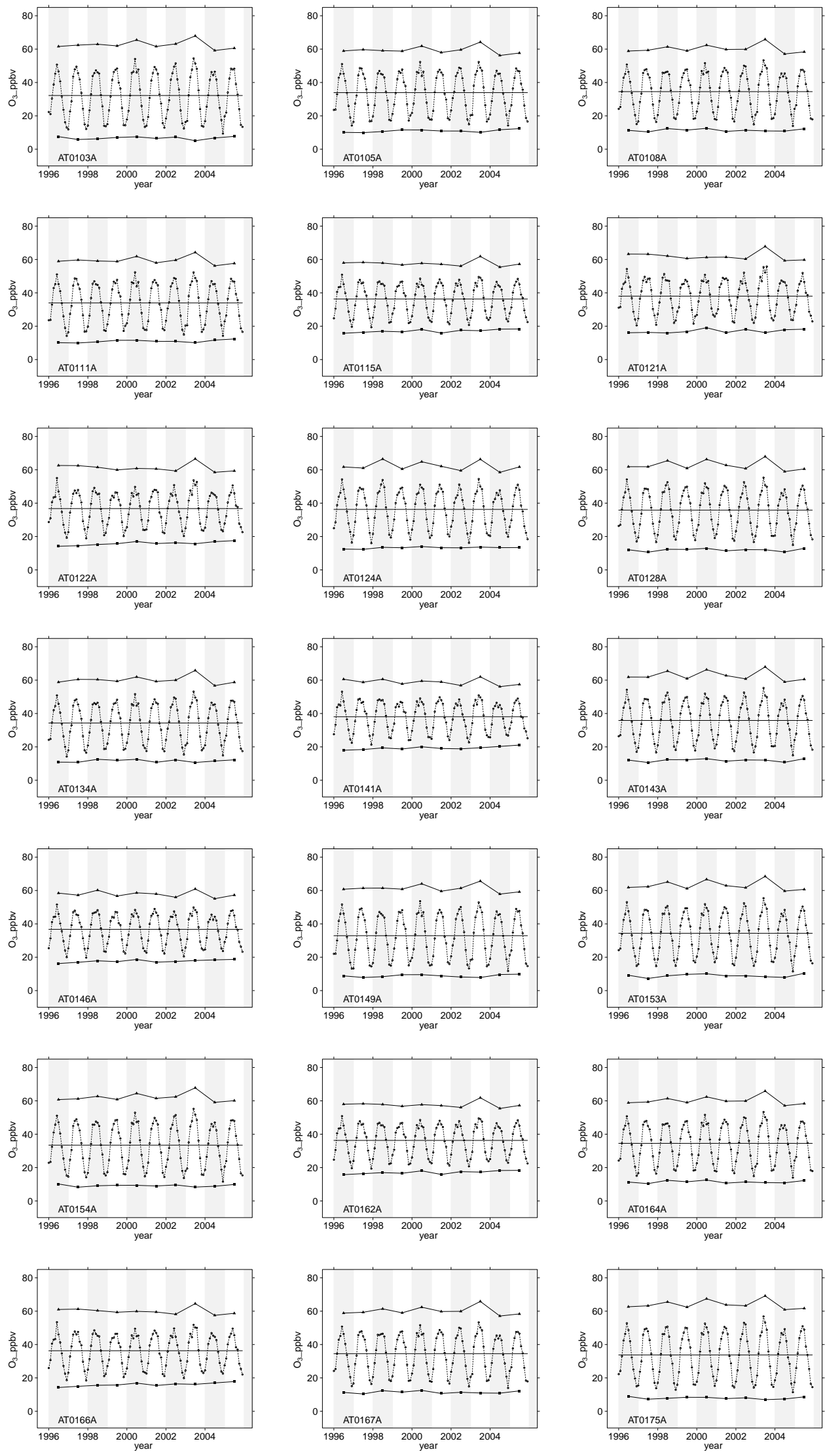

Figure B1: continued. Loess trends of CHIMERE simulated monthly mean $\mathrm{O}_{3}$ 

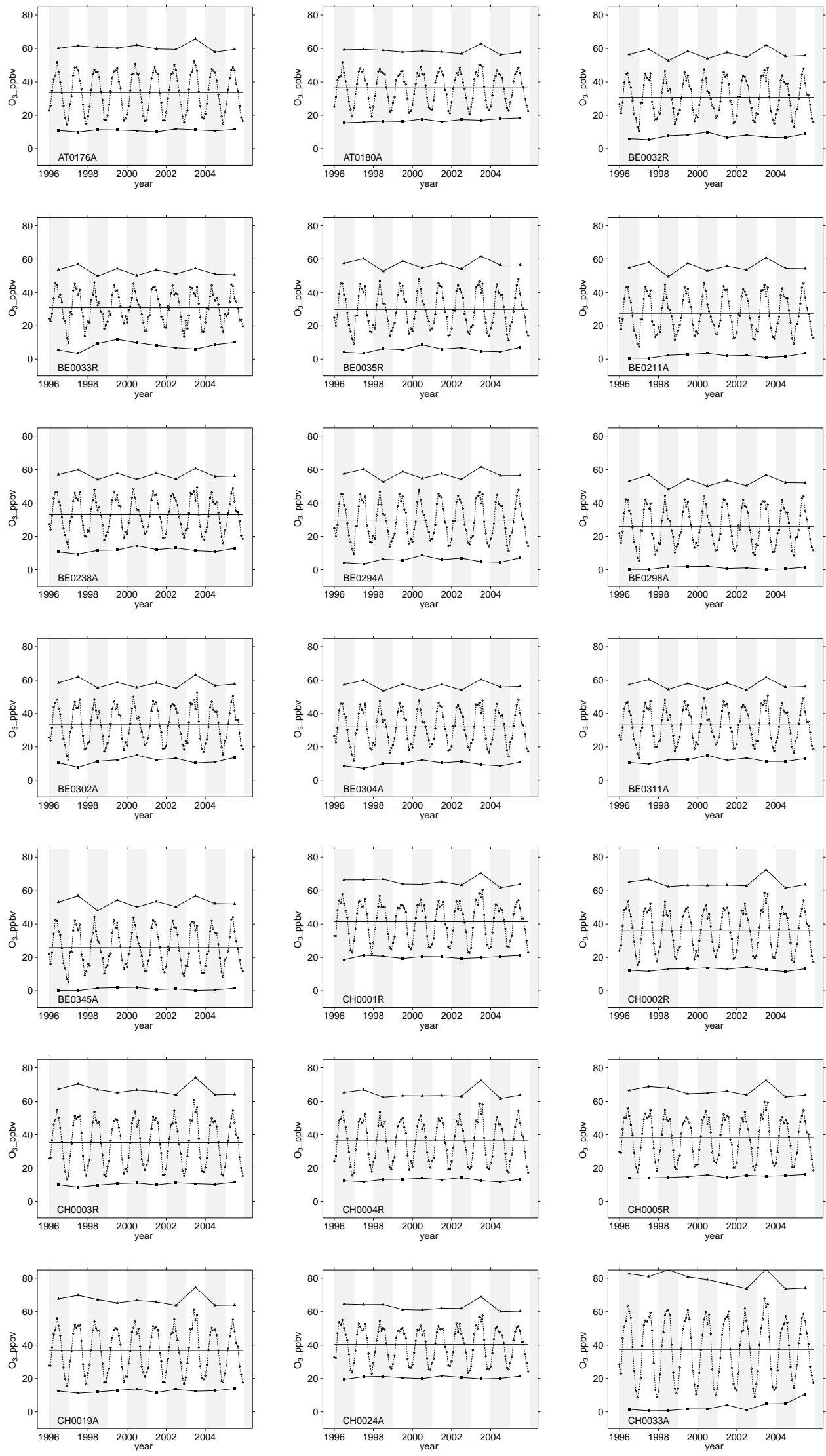

Figure B1: continued. Loess trends of CHIMERE simulated monthly mean $\mathrm{O}_{3}$ 

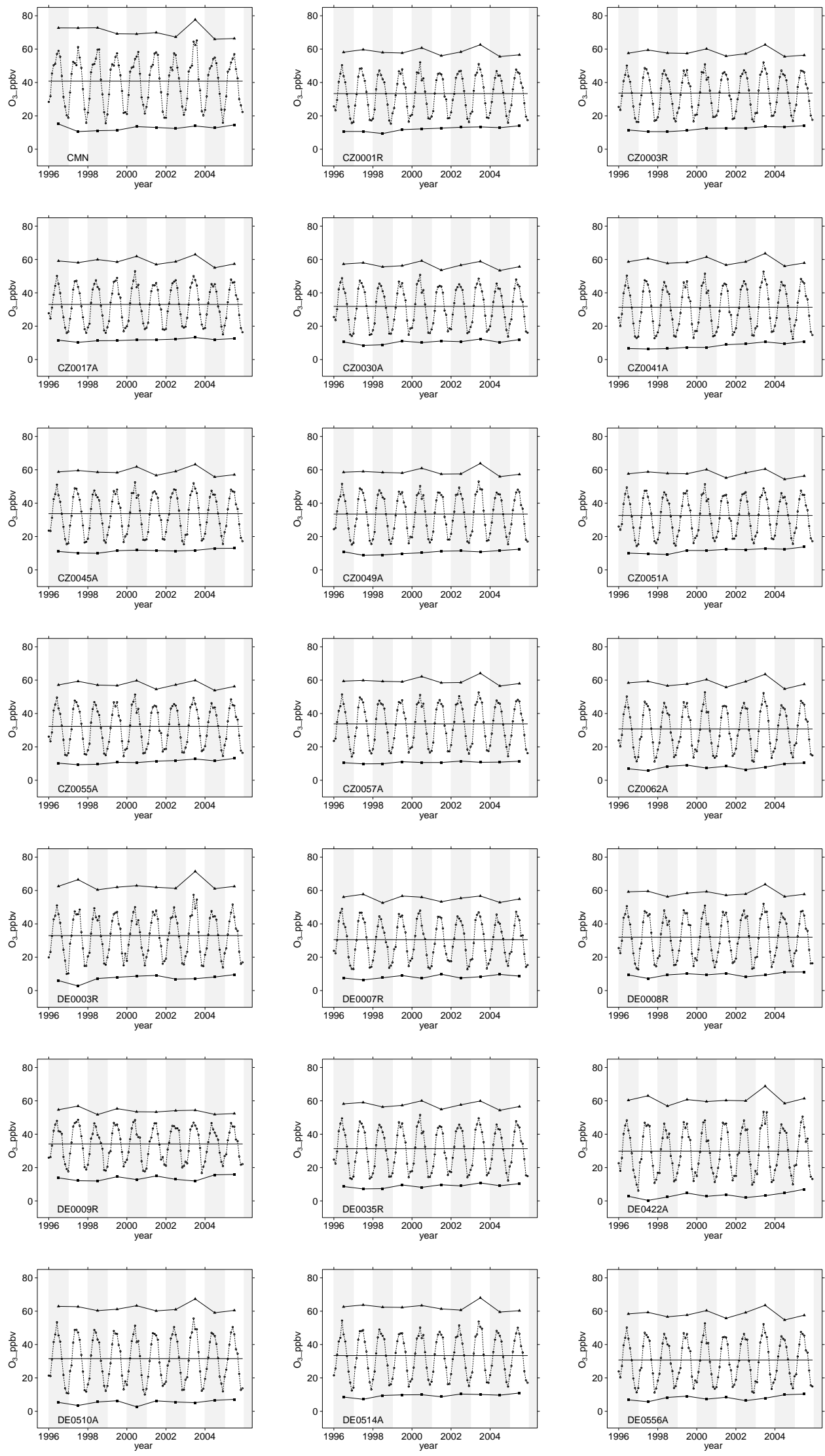

Figure B1: continued. Loess trends of CHIMERE simulated monthly mean $\mathrm{O}_{3}$ 

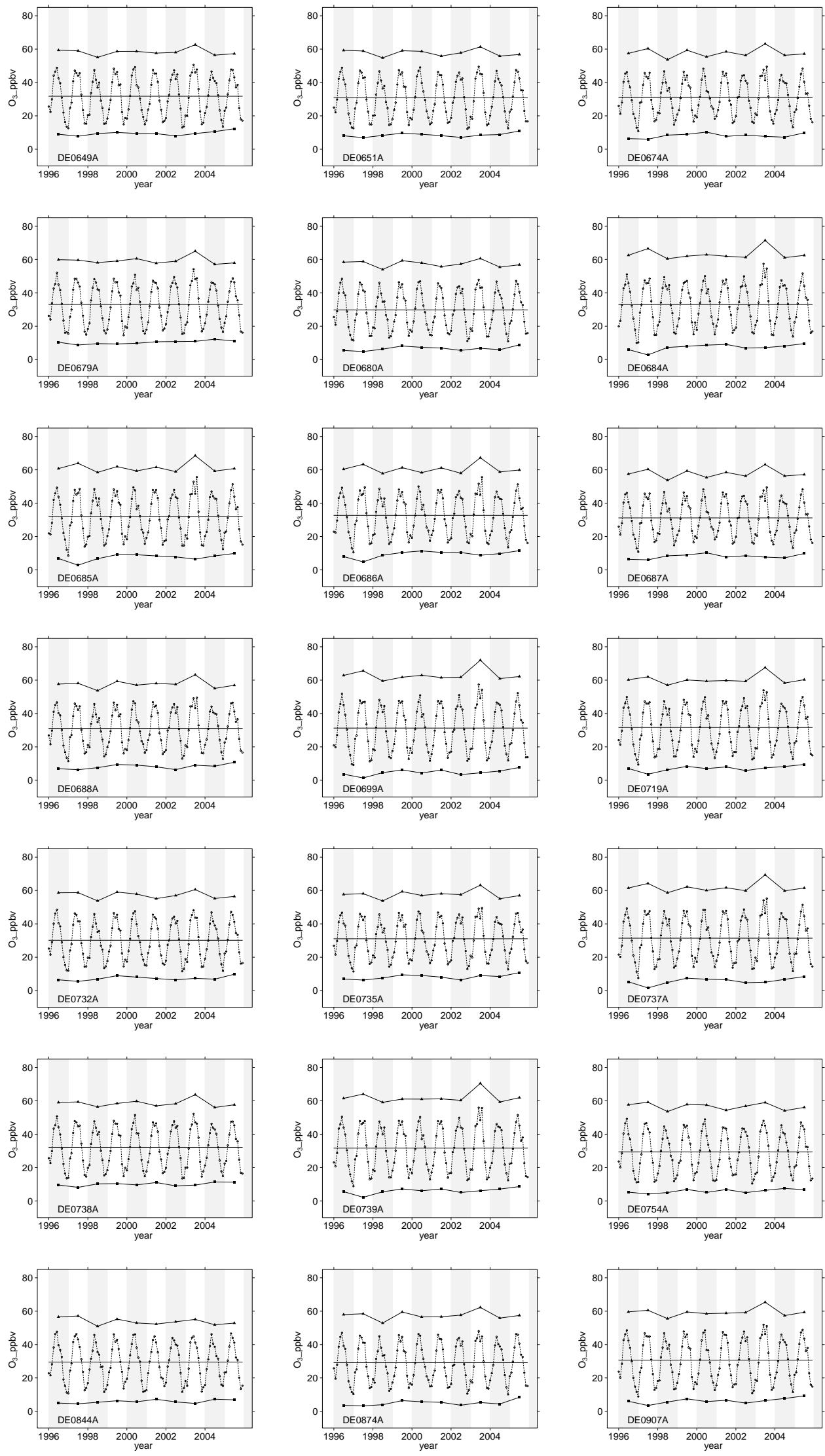

Figure B1: continued. Loess trends of CHIMERE simulated monthly mean $\mathrm{O}_{3}$ 

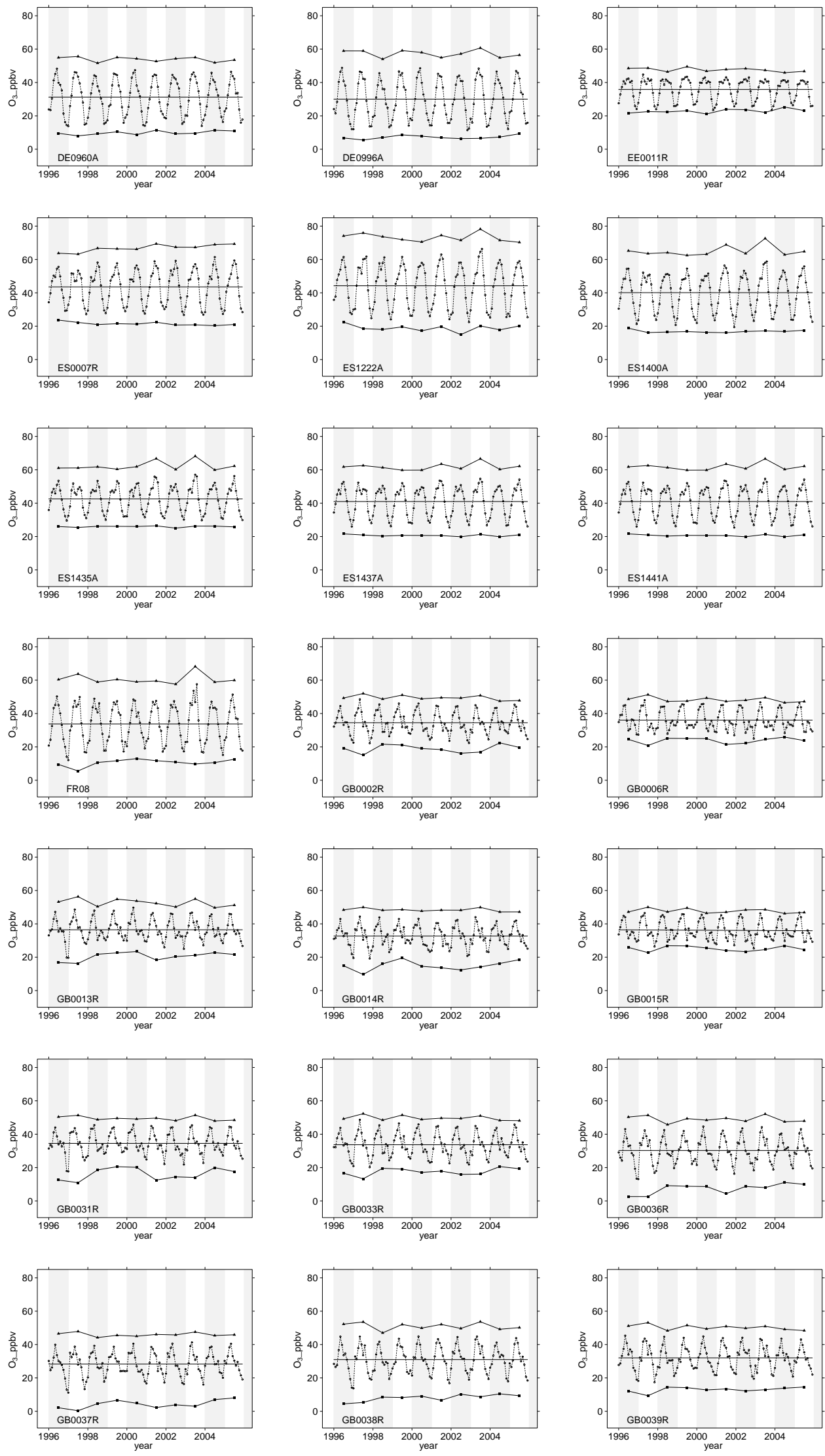

Figure B1: continued. Loess trends of CHIMERE simulated monthly mean $\mathrm{O}_{3}$ 

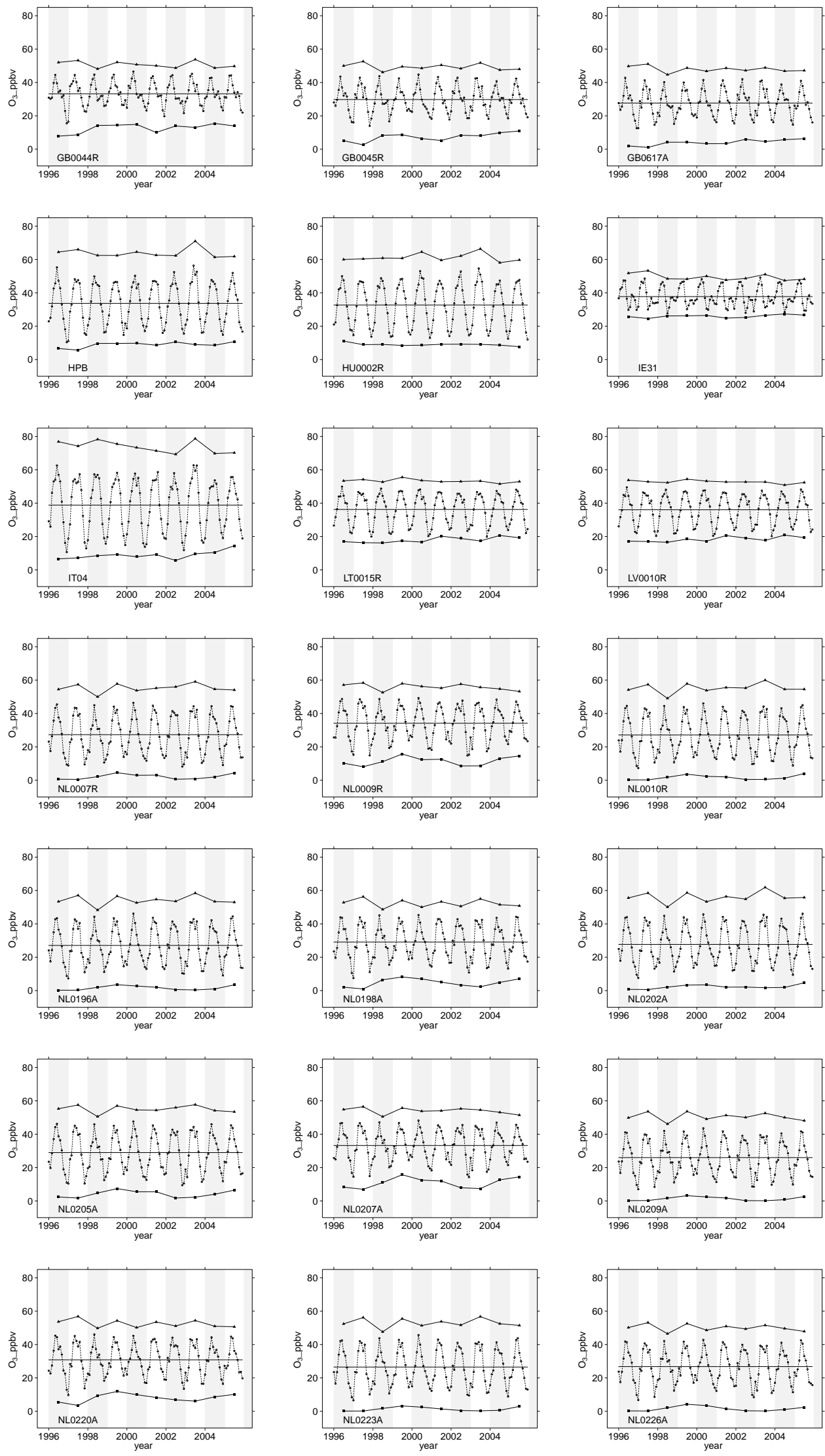

Figure B1: continued. Loess trends of CHIMERE simulated monthly mean $\mathrm{O}_{3}$ 

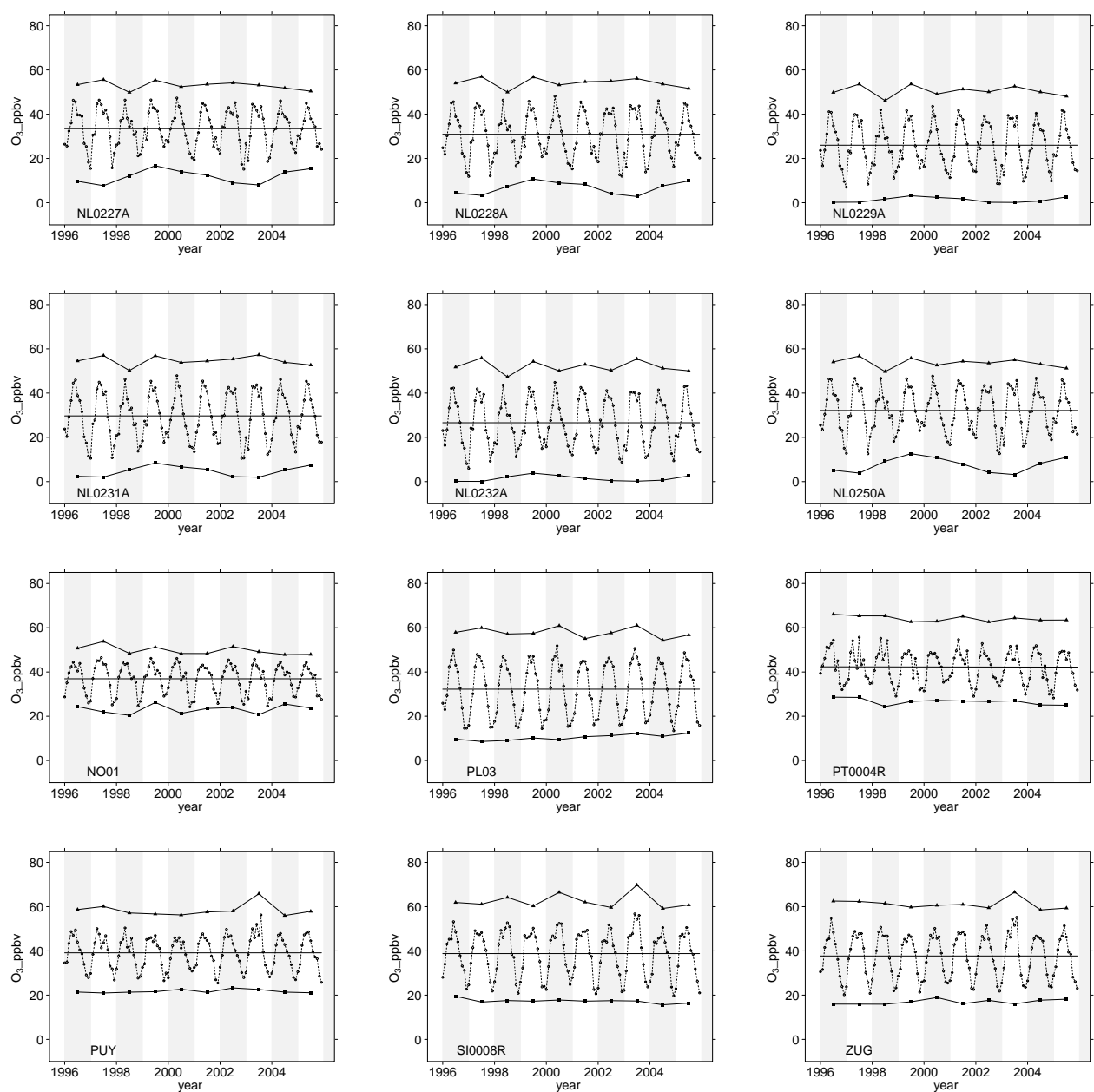

Figure B1: continued. Loess trends of CHIMERE simulated monthly mean $\mathrm{O}_{3}$ 
Table B1: Quantification of annual CHIMERE model trends (ppbv/yr and \%/yr) in mean $\mathrm{O}_{3}$ 1996-2005. 95\% confidence interval given in brackets. Sig. = significance $* * *$ significant at $99.9 \%$ level, ${ }^{* *}$ significant at $99.0 \%$ level, ${ }^{*}$ significant at $95.0 \%$ level, + significant at $90.0 \%$ level. ${ }^{\ddagger} 2 \sigma$ error. ${ }^{\diamond}$ significant trends only

\begin{tabular}{|c|c|c|c|c|c|c|}
\hline ID & Trend (ppbv/yr) & Sig. & $\mathrm{P}$-value & Trend $(\% / y r)$ & Sig. & $\mathrm{P}$-value \\
\hline AT0002R & $0.07(-0.03-0.18)$ & & 0.158 & $0.31(-0.09-0.70)$ & & 0.140 \\
\hline AT0004R & $0.07(-0.03-0.17)$ & & 0.199 & $0.18(-0.09-0.51)$ & & 0.207 \\
\hline AT0005R & $0.04(-0.09-0.16)$ & & 0.586 & $0.20(-0.16-0.59)$ & & 0.345 \\
\hline AT0034R & $0.04(-0.07-0.15)$ & & 0.528 & $0.18(-0.16-0.49)$ & & 0.261 \\
\hline AT0044A & $0.11(-0.02-0.22)$ & + & 0.077 & $0.49(0.04-0.91)$ & $*$ & 0.026 \\
\hline AT0052A & $-0.01(-0.13-0.09)$ & & 0.838 & $0.00(-0.31-0.30)$ & & 0.993 \\
\hline AT0054A & $0.09(0.01-0.19)$ & + & 0.055 & $0.52(0.12-0.96)$ & $*$ & 0.010 \\
\hline AT0058A & $0.00(-0.11-0.11)$ & & 1.000 & $0.05(-0.27-0.37)$ & & 0.768 \\
\hline AT0064A & $0.02(-0.11-0.14)$ & & 0.707 & $0.04(-0.34-0.42)$ & & 0.842 \\
\hline AT0069A & $0.11(0.01-0.20)$ & $*$ & 0.044 & $0.42(0.02-0.83)$ & $*$ & 0.023 \\
\hline AT0073A & $0.10(0.01-0.19)$ & $*$ & 0.049 & $0.46(0.15-0.81)$ & $* *$ & 0.005 \\
\hline AT0079A & $0.04(-0.07-0.15)$ & & 0.528 & $0.18(-0.16-0.49)$ & & 0.261 \\
\hline AT0080A & $0.05(-0.04-0.13)$ & & 0.310 & $0.21(-0.04-0.51)$ & & 0.113 \\
\hline AT0086A & $0.06(-0.03-0.14)$ & & 0.247 & $0.26(-0.01-0.53)$ & + & 0.068 \\
\hline AT0089A & $0.02(-0.11-0.13)$ & & 0.779 & $0.05(-0.33-0.38)$ & & 0.754 \\
\hline AT0094A & $-0.01(-0.13-0.09)$ & & 0.838 & $0.00(-0.31-0.30)$ & & 0.993 \\
\hline AT0095A & $0.11(0.00-0.22)$ & + & 0.052 & $0.56(0.13-1.02)$ & $*$ & 0.012 \\
\hline AT0096A & $0.08(-0.08-0.21)$ & & 0.312 & $0.23(-0.18-0.68)$ & & 0.246 \\
\hline AT0101A & $0.16(0.06-0.26)$ & $* *$ & 0.003 & $0.67(0.38-1.01)$ & $* * *$ & $<0.001$ \\
\hline AT0102A & $0.11(0.00-0.22)$ & + & 0.052 & $0.56(0.13-1.02)$ & $*$ & 0.012 \\
\hline AT0103A & $0.09(-0.03-0.21)$ & & 0.114 & $0.35(-0.08-0.81)$ & & 0.119 \\
\hline AT0105A & $0.10(-0.02-0.22)$ & & 0.101 & $0.39(0.02-0.80)$ & $*$ & 0.035 \\
\hline AT0108A & $0.04(-0.08-0.17)$ & & 0.386 & $0.17(-0.23-0.56)$ & & 0.343 \\
\hline AT0111A & $0.10(-0.02-0.22)$ & & 0.101 & $0.39(0.02-0.80)$ & $*$ & 0.035 \\
\hline AT0115A & $0.07(-0.03-0.15)$ & & 0.211 & $0.26(-0.03-0.52)$ & + & 0.099 \\
\hline AT0121A & $-0.01(-0.15-0.11)$ & & 0.842 & $-0.01(-0.41-0.36)$ & & 0.939 \\
\hline AT0122A & $0.08(-0.04-0.19)$ & & 0.199 & $0.25(-0.11-0.59)$ & & 0.166 \\
\hline AT0124A & $-0.04(-0.17-0.06)$ & & 0.417 & $-0.14(-0.50-0.23)$ & & 0.438 \\
\hline AT0128A & $-0.01(-0.15-0.11)$ & & 0.796 & $0.00(-0.42-0.35)$ & & 0.996 \\
\hline AT0134A & $0.07(-0.05-0.18)$ & & 0.235 & $0.19(-0.14-0.57)$ & & 0.270 \\
\hline AT0141A & $0.04(-0.07-0.15)$ & & 0.528 & $0.18(-0.16-0.49)$ & & 0.261 \\
\hline AT0143A & $-0.01(-0.15-0.11)$ & & 0.796 & $0.00(-0.42-0.35)$ & & 0.996 \\
\hline AT0146A & $0.03(-0.08-0.13)$ & & 0.589 & $0.11(-0.19-0.42)$ & & 0.482 \\
\hline AT0149A & $0.11(-0.02-0.22)$ & + & 0.077 & $0.49(0.04-0.91)$ & $*$ & 0.026 \\
\hline AT0153A & $0.03(-0.10-0.15)$ & & 0.599 & $0.23(-0.22-0.60)$ & & 0.217 \\
\hline AT0154A & $0.06(-0.05-0.17)$ & & 0.262 & $0.23(-0.14-0.60)$ & & 0.174 \\
\hline AT0162A & $0.07(-0.03-0.15)$ & & 0.211 & $0.26(-0.03-0.52)$ & + & 0.099 \\
\hline AT0164A & $0.04(-0.08-0.17)$ & & 0.386 & $0.17(-0.23-0.56)$ & & 0.343 \\
\hline AT0166A & $0.07(-0.03-0.17)$ & & 0.199 & $0.18(-0.09-0.51)$ & & 0.207 \\
\hline AT0167A & $0.04(-0.08-0.17)$ & & 0.386 & $0.17(-0.23-0.56)$ & & 0.343 \\
\hline AT0175A & $0.01(-0.10-0.11)$ & & 0.810 & $0.21(-0.20-0.53)$ & & 0.211 \\
\hline AT0176A & $0.05(-0.04-0.13)$ & & 0.310 & $0.21(-0.04-0.51)$ & & 0.113 \\
\hline
\end{tabular}


Table B1: continued. Quantification of annual CHIMERE model trends (ppbv/yr and $\% / y r$ ) in mean $\mathrm{O}_{3}$ 1996-2005. 95\% confidence interval given in brackets. Sig. = significance ${ }^{* * *}$ significant at $99.9 \%$ level, ${ }^{* *}$ significant at $99.0 \%$ level, ${ }^{*}$ significant at $95.0 \%$ level, + significant at $90.0 \%$ level. ${ }^{\ddagger} 2 \sigma$ error. ${ }^{\diamond}$ significant trends only

\begin{tabular}{|c|c|c|c|c|c|c|}
\hline ID & Trend (ppbv/yr) & Sig. & P-value & Trend (\%/yr) & Sig. & $\mathrm{P}$-value \\
\hline AT0180A & $0.07(-0.03-0.16)$ & & 0.174 & $0.22(-0.05-0.51)$ & & 0.138 \\
\hline BE0032R & $0.07(-0.09-0.19)$ & & 0.350 & $0.55(-0.16-1.10)$ & & 0.110 \\
\hline BE0033R & $-0.04(-0.19-0.15)$ & & 0.683 & $0.18(-0.58-0.99)$ & & 0.647 \\
\hline BE0035R & $0.05(-0.10-0.17)$ & & 0.449 & $0.72(-0.11-1.41)$ & + & 0.063 \\
\hline BE0211A & $0.05(-0.10-0.18)$ & & 0.471 & $0.94(-0.17-2.08)$ & + & 0.093 \\
\hline BE0238A & $0.05(-0.09-0.16)$ & & 0.401 & $0.32(-0.19-0.72)$ & & 0.164 \\
\hline BE0294A & $0.05(-0.10-0.17)$ & & 0.449 & $0.72(-0.11-1.41)$ & + & 0.063 \\
\hline BE0298A & $0.04(-0.11-0.19)$ & & 0.583 & $0.80(-0.52-2.08)$ & & 0.188 \\
\hline BE0302A & $0.05(-0.09-0.17)$ & & 0.404 & $0.35(-0.12-0.77)$ & & 0.117 \\
\hline BE0304A & $0.06(-0.07-0.17)$ & & 0.391 & $0.36(-0.24-0.84)$ & & 0.194 \\
\hline BE0311A & $0.04(-0.09-0.16)$ & & 0.482 & $0.34(-0.26-0.78)$ & & 0.176 \\
\hline BE0345A & $0.04(-0.11-0.19)$ & & 0.583 & $0.80(-0.52-2.08)$ & & 0.188 \\
\hline CH0001R & $-0.06(-0.23-0.09)$ & & 0.371 & $-0.13(-0.58-0.34)$ & & 0.549 \\
\hline $\mathrm{CH} 0002 \mathrm{R}$ & $-0.06(-0.17-0.05)$ & & 0.314 & $-0.10(-0.52-0.31)$ & & 0.565 \\
\hline CH0003R & $-0.01(-0.14-0.11)$ & & 0.860 & $0.20(-0.18-0.70)$ & & 0.338 \\
\hline CH0004R & $-0.06(-0.17-0.05)$ & & 0.314 & $-0.10(-0.52-0.31)$ & & 0.565 \\
\hline CH0005R & $-0.02(-0.17-0.11)$ & & 0.779 & $0.04(-0.42-0.54)$ & & 0.874 \\
\hline CH0019A & $-0.03(-0.16-0.10)$ & & 0.713 & $0.17(-0.22-0.61)$ & & 0.430 \\
\hline $\mathrm{CH} 0024 \mathrm{~A}$ & $-0.12(-0.25-0.02)$ & + & 0.091 & $-0.33(-0.67-0.06)$ & + & 0.060 \\
\hline CH0033A & $0.07(-0.18-0.27)$ & & 0.528 & $0.90(0.00-2.11)$ & $*$ & 0.035 \\
\hline CMN & $-0.12(-0.26-0.01)$ & + & 0.074 & $-0.19(-0.51-0.22)$ & & 0.274 \\
\hline CZ0001R & $0.20(0.10-0.30)$ & $* * *$ & $<0.001$ & $0.94(0.54-1.33)$ & $* * *$ & $<0.001$ \\
\hline CZ0003R & $0.21(0.11-0.31)$ & $* * *$ & $<0.001$ & $0.91(0.59-1.31)$ & $* * *$ & $<0.001$ \\
\hline CZ0017A & $0.16(0.03-0.27)$ & $* *$ & 0.006 & $0.74(0.33-1.09)$ & $* * *$ & $<0.001$ \\
\hline CZ0030A & $0.13(0.02-0.22)$ & $*$ & 0.020 & $0.77(0.33-1.21)$ & $* * *$ & 0.001 \\
\hline CZ0041A & $0.32(0.19-0.43)$ & $* * *$ & $<0.001$ & $1.84(1.20-2.44)$ & $* * *$ & $<0.001$ \\
\hline CZ0045A & $0.17(0.06-0.27)$ & $* *$ & 0.005 & $0.72(0.37-1.12)$ & $* * *$ & $<0.001$ \\
\hline CZ0049A & $0.15(0.05-0.24)$ & $* *$ & 0.007 & $0.72(0.32-1.15)$ & & $<0.001$ \\
\hline CZ0051A & $0.17(0.06-0.27)$ & $* *$ & 0.002 & $0.87(0.45-1.32)$ & $* * *$ & $<0.001$ \\
\hline CZ0055A & $0.18(0.07-0.28)$ & $* *$ & 0.001 & $0.96(0.53-1.46)$ & $* * *$ & $<0.001$ \\
\hline CZ0057A & $0.10(0.01-0.19)$ & $*$ & 0.049 & $0.46(0.15-0.81)$ & $* *$ & 0.005 \\
\hline CZ0062A & $0.19(0.04-0.32)$ & $*$ & 0.012 & $1.09(0.40-1.86)$ & $* *$ & 0.002 \\
\hline DE0003R & $0.06(-0.06-0.18)$ & & 0.314 & $0.62(0.14-1.22)$ & $* *$ & 0.009 \\
\hline DE0007R & $-0.02(-0.14-0.10)$ & & 0.810 & $0.32(-0.20-0.96)$ & & 0.264 \\
\hline DE0008R & $0.11(-0.01-0.24)$ & & 0.102 & $0.61(0.15-1.17)$ & $*$ & 0.014 \\
\hline DE0009R & $0.00(-0.15-0.13)$ & & 0.986 & $0.20(-0.30-0.73)$ & & 0.425 \\
\hline DE0035R & $0.12(0.01-0.22)$ & $*$ & 0.027 & $0.81(0.36-1.36)$ & $* * *$ & 0.001 \\
\hline DE0422A & $0.20(0.04-0.35)$ & $*$ & 0.013 & $1.40(0.50-2.60)$ & $* *$ & 0.002 \\
\hline DE0510A & $0.10(-0.02-0.22)$ & & 0.102 & $0.50(-0.04-1.13)$ & + & 0.086 \\
\hline DE0514A & $0.11(0.01-0.20)$ & $*$ & 0.044 & $0.42(0.02-0.83)$ & $*$ & 0.023 \\
\hline DE0556A & $0.19(0.04-0.32)$ & $*$ & 0.012 & $1.09(0.40-1.86)$ & $* *$ & 0.002 \\
\hline DE0649A & $0.14(0.01-0.27)$ & $*$ & 0.045 & $0.73(0.17-1.41)$ & $* *$ & 0.008 \\
\hline
\end{tabular}


Table B1: continued. Quantification of annual CHIMERE model trends (ppbv/yr and $\% / y r$ ) in mean $\mathrm{O}_{3}$ 1996-2005. 95\% confidence interval given in brackets. Sig. = significance ${ }^{* * *}$ significant at $99.9 \%$ level, ${ }^{* *}$ significant at $99.0 \%$ level, ${ }^{*}$ significant at $95.0 \%$ level, + significant at $90.0 \%$ level. ${ }^{\ddagger} 2 \sigma$ error. ${ }^{\diamond}$ significant trends only

\begin{tabular}{|c|c|c|c|c|c|c|}
\hline ID & Trend (ppbv/yr) & Sig. & P-value & Trend $(\% / y r)$ & Sig. & $\mathrm{P}$-value \\
\hline$\overline{\mathrm{DE} 0651 \mathrm{~A}}$ & $0.10(-0.02-0.22)$ & & 0.101 & $0.61(0.11-1.18)$ & $*$ & 0.012 \\
\hline DE0674A & $0.08(-0.06-0.20)$ & & 0.221 & $0.62(-0.01-1.09)$ & + & 0.054 \\
\hline DE0679A & $0.11(0.01-0.22)$ & + & 0.054 & $0.64(0.17-1.10)$ & $* *$ & 0.007 \\
\hline DE0680A & $0.10(-0.03-0.22)$ & & 0.129 & $0.85(0.19-1.55)$ & * & 0.013 \\
\hline DE0684A & $0.06(-0.06-0.18)$ & & 0.314 & $0.62(0.14-1.22)$ & $* *$ & 0.009 \\
\hline DE0685A & $0.08(-0.06-0.24)$ & & 0.246 & $0.72(0.06-1.50)$ & $*$ & 0.027 \\
\hline DE0686A & $0.07(-0.07-0.21)$ & & 0.305 & $0.57(-0.02-1.12)$ & + & 0.053 \\
\hline DE0687A & $0.08(-0.06-0.20)$ & & 0.221 & $0.62(-0.01-1.09)$ & + & 0.054 \\
\hline DE0688A & $0.15(0.02-0.27)$ & $*$ & 0.024 & $0.89(0.25-1.71)$ & $* *$ & 0.005 \\
\hline DE0699A & $0.13(-0.01-0.27)$ & + & 0.075 & $0.96(0.24-1.67)$ & $* *$ & 0.005 \\
\hline DE0719A & $0.13(-0.01-0.27)$ & + & 0.078 & $1.04(0.26-1.78)$ & $* *$ & 0.007 \\
\hline DE0732A & $0.11(-0.01-0.23)$ & + & 0.089 & $0.80(0.20-1.41)$ & $*$ & 0.010 \\
\hline DE0735A & $0.15(0.02-0.27)$ & $*$ & 0.024 & $0.89(0.25-1.71)$ & $* *$ & 0.005 \\
\hline DE0737A & $0.11(-0.02-0.27)$ & & 0.137 & $1.02(0.12-1.94)$ & * & 0.017 \\
\hline DE0738A & $0.11(-0.01-0.24)$ & + & 0.092 & $0.65(0.18-1.21)$ & $* *$ & 0.010 \\
\hline DE0739A & $0.11(-0.03-0.26)$ & & 0.164 & $0.94(0.18-1.64)$ & $*$ & 0.010 \\
\hline DE0754A & $0.03(-0.11-0.16)$ & & 0.676 & $0.60(-0.05-1.31)$ & + & 0.100 \\
\hline DE0844A & $0.02(-0.14-0.15)$ & & 0.806 & $0.41(-0.25-1.11)$ & & 0.280 \\
\hline DE0874A & $0.16(0.02-0.27)$ & $*$ & 0.020 & $1.46(0.47-2.57)$ & $* * *$ & 0.001 \\
\hline DE0907A & $0.17(0.03-0.32)$ & $*$ & 0.025 & $1.27(0.45-2.10)$ & $* *$ & 0.002 \\
\hline DE0960A & $0.00(-0.13-0.12)$ & & 0.989 & $0.26(-0.27-0.75)$ & & 0.336 \\
\hline DE0996A & $0.07(-0.05-0.20)$ & & 0.253 & $0.56(0.05-1.16)$ & $*$ & 0.044 \\
\hline EE0011R & $-0.02(-0.08-0.04)$ & & 0.589 & $0.00(-0.17-0.19)$ & & 0.975 \\
\hline ES0007R & $0.11(-0.02-0.23)$ & + & 0.086 & $0.18(-0.13-0.47)$ & & 0.240 \\
\hline $\mathrm{ES} 1222 \mathrm{~A}$ & $-0.08(-0.23-0.08)$ & & 0.312 & $-0.16(-0.67-0.30)$ & & 0.476 \\
\hline ES1400A & $0.03(-0.09-0.15)$ & & 0.555 & $0.09(-0.25-0.38)$ & & 0.561 \\
\hline ES1435A & $-0.01(-0.08-0.08)$ & & 0.821 & $0.01(-0.19-0.20)$ & & 0.903 \\
\hline ES1437A & $-0.02(-0.13-0.09)$ & & 0.720 & $-0.03(-0.31-0.23)$ & & 0.863 \\
\hline ES1441A & $-0.02(-0.13-0.09)$ & & 0.720 & $-0.03(-0.31-0.23)$ & & 0.863 \\
\hline FR08 & $0.06(-0.07-0.19)$ & & 0.374 & $0.45(-0.12-0.95)$ & & 0.105 \\
\hline GB0002R & $-0.04(-0.16-0.08)$ & & 0.425 & $-0.12(-0.45-0.31)$ & & 0.618 \\
\hline GB0006R & $-0.08(-0.17-0.03)$ & & 0.121 & $-0.18(-0.49-0.12)$ & & 0.224 \\
\hline GB0013R & $-0.07(-0.18-0.05)$ & & 0.246 & $-0.06(-0.44-0.33)$ & & 0.768 \\
\hline GB0014R & $0.06(-0.06-0.18)$ & & 0.350 & $0.27(-0.16-0.81)$ & & 0.246 \\
\hline GB0015R & $-0.09(-0.18-0.00)$ & $*$ & 0.039 & $-0.25(-0.50-0.01)$ & + & 0.052 \\
\hline GB0031R & $-0.03(-0.18-0.12)$ & & 0.644 & $-0.11(-0.64-0.47)$ & & 0.683 \\
\hline GB0033R & $0.02(-0.10-0.14)$ & & 0.680 & $0.14(-0.26-0.62)$ & & 0.485 \\
\hline GB0036R & $0.14(-0.02-0.30)$ & + & 0.097 & $0.75(-0.09-1.89)$ & + & 0.072 \\
\hline GB0037R & $0.19(0.03-0.36)$ & $*$ & 0.024 & $1.46(0.63-2.45)$ & $* *$ & 0.001 \\
\hline GB0038R & $0.11(-0.02-0.24)$ & & 0.140 & $1.19(0.36-2.10)$ & $* *$ & 0.005 \\
\hline GB0039R & $-0.04(-0.17-0.11)$ & & 0.583 & $0.03(-0.48-0.61)$ & & 0.899 \\
\hline GB0044R & $0.01(-0.14-0.17)$ & & 0.899 & $0.36(-0.35-1.09)$ & & 0.268 \\
\hline
\end{tabular}


Table B1: continued. Quantification of annual CHIMERE model trends (ppbv/yr and \%/yr) in mean $\mathrm{O}_{3}$ 1996-2005. 95\% confidence interval given in brackets. Sig. = significance ${ }^{* * *}$ significant at $99.9 \%$ level, ${ }^{* *}$ significant at $99.0 \%$ level, ${ }^{*}$ significant at $95.0 \%$ level, + significant at $90.0 \%$ level. ${ }^{\ddagger} 2 \sigma$ error. ${ }^{\diamond}$ significant trends only

\begin{tabular}{|c|c|c|c|c|c|c|}
\hline ID & Trend (ppbv/yr) & Sig. & $\mathrm{P}$-value & Trend (\%/yr) & Sig. & $\mathrm{P}$-value \\
\hline GB0045R & $0.12(-0.02-0.26)$ & & 0.117 & $1.25(0.18-2.34)$ & $*$ & 0.021 \\
\hline GB0617A & $0.10(-0.04-0.26)$ & & 0.144 & $1.48(0.39-2.56)$ & $* *$ & 0.009 \\
\hline HPB & $0.06(-0.05-0.17)$ & & 0.268 & $0.42(-0.02-0.90)$ & + & 0.072 \\
\hline HU0002R & $-0.02(-0.13-0.08)$ & & 0.660 & $-0.03(-0.50-0.37)$ & & 0.845 \\
\hline IE31 & $-0.08(-0.14-0.01)$ & + & 0.064 & $-0.17(-0.34-0.05)$ & + & 0.086 \\
\hline IT04 & $0.00(-0.23-0.20)$ & & 0.993 & $0.21(-0.38-0.90)$ & & 0.462 \\
\hline LT0015R & $0.00(-0.09-0.09)$ & & 0.986 & $0.06(-0.21-0.32)$ & & 0.637 \\
\hline LV0010R & $0.01(-0.07-0.09)$ & & 0.824 & $0.11(-0.16-0.36)$ & & 0.427 \\
\hline NL0007R & $0.06(-0.10-0.22)$ & & 0.474 & $0.48(-0.59-1.74)$ & & 0.406 \\
\hline NL0009R & $0.00(-0.17-0.16)$ & & 0.978 & $0.24(-0.45-1.02)$ & & 0.502 \\
\hline NL0010R & $0.08(-0.08-0.22)$ & & 0.284 & $0.66(-0.34-1.74)$ & & 0.187 \\
\hline NL0196A & $0.05(-0.11-0.19)$ & & 0.514 & $0.36(-0.68-1.39)$ & & 0.485 \\
\hline NL0198A & $-0.02(-0.18-0.17)$ & & 0.877 & $0.56(-0.37-1.57)$ & & 0.255 \\
\hline NL0202A & $0.10(-0.06-0.23)$ & & 0.187 & $1.00(-0.10-2.15)$ & + & 0.060 \\
\hline NL0205A & $0.03(-0.15-0.19)$ & & 0.758 & $0.62(-0.29-1.60)$ & & 0.157 \\
\hline NL0207A & $0.01(-0.18-0.19)$ & & 0.953 & $0.27(-0.45-1.12)$ & & 0.528 \\
\hline NL0209A & $0.00(-0.18-0.17)$ & & 0.989 & $-0.04(-1.22-1.22)$ & & 0.942 \\
\hline NL0220A & $-0.04(-0.19-0.15)$ & & 0.683 & $0.18(-0.58-0.99)$ & & 0.647 \\
\hline NL0223A & $0.03(-0.14-0.19)$ & & 0.727 & $0.68(-0.64-1.99)$ & & 0.338 \\
\hline NL0226A & $-0.05(-0.24-0.13)$ & & 0.605 & $0.15(-1.22-1.56)$ & & 0.845 \\
\hline NL0227A & $-0.03(-0.20-0.17)$ & & 0.741 & $0.10(-0.54-0.95)$ & & 0.754 \\
\hline NL0228A & $0.03(-0.17-0.22)$ & & 0.765 & $0.57(-0.33-1.49)$ & & 0.214 \\
\hline NL0229A & $0.00(-0.18-0.17)$ & & 0.989 & $-0.04(-1.22-1.22)$ & & 0.942 \\
\hline NL0231A & $0.02(-0.17-0.20)$ & & 0.782 & $0.49(-0.35-1.39)$ & & 0.242 \\
\hline NL0232A & $0.00(-0.16-0.16)$ & & 0.960 & $0.43(-0.69-1.75)$ & & 0.441 \\
\hline NL0250A & $-0.04(-0.22-0.15)$ & & 0.720 & $0.24(-0.49-1.05)$ & & 0.528 \\
\hline NO01 & $-0.05(-0.15-0.05)$ & & 0.449 & $-0.05(-0.31-0.24)$ & & 0.720 \\
\hline PL03 & $0.19(0.07-0.30)$ & $* *$ & 0.001 & $1.08(0.59-1.62)$ & $* * *$ & $<0.001$ \\
\hline PT0004R & $-0.14(-0.32-0.06)$ & + & 0.100 & $-0.32(-0.72-0.22)$ & & 0.143 \\
\hline PUY & $-0.07(-0.16-0.06)$ & & 0.318 & $-0.15(-0.45-0.18)$ & & 0.401 \\
\hline SI0008R & $-0.08(-0.23-0.06)$ & & 0.231 & $-0.23(-0.68-0.23)$ & & 0.325 \\
\hline ZUG & $0.02(-0.11-0.13)$ & & 0.779 & $0.05(-0.33-0.38)$ & & 0.754 \\
\hline $\begin{array}{c}\text { European } \\
\text { average }^{\ddagger}\end{array}$ & $0.05 \pm 0.01 \mathrm{ppbv} / \mathrm{yr}$ & & & $0.41 \pm 0.05 \% / \mathrm{yr}$ & & \\
\hline Range $^{\diamond}$ & -0.14 to $0.32 \mathrm{ppbv} / \mathrm{yr}$ & & & -4.11 to $6.05 \% / y r$ & & \\
\hline
\end{tabular}


Table B2: Quantification of annual CHIMERE model trends (ppbv/yr and \%/yr) in $\mathrm{O}_{3}$ $5^{\text {th }}$ percentiles $1996-2005.95 \%$ confidence interval given in brackets. Sig. $=$ significance *** significant at $99.9 \%$ level, ** significant at $99.0 \%$ level, ${ }^{*}$ significant at $95.0 \%$ level, + significant at $90.0 \%$ level. ${ }^{\ddagger} 2 \sigma$ error. ${ }^{\diamond}$ significant trends only

\begin{tabular}{|c|c|c|c|c|c|c|}
\hline ID & Trend (ppbv/yr) & Sig. & P-value & Trend (\%/yr) & Sig. & $\mathrm{P}$-value \\
\hline AT0002R & $0.17(0.04-0.29)$ & $* *$ & 0.007 & $1.05(-0.15-2.18)$ & + & 0.058 \\
\hline AT0004R & $0.10(-0.04-0.22)$ & & 0.125 & $0.53(-0.22-1.15)$ & & 0.129 \\
\hline AT0005R & $0.13(0.00-0.27)$ & $*$ & 0.048 & $0.59(-0.04-1.21)$ & + & 0.068 \\
\hline AT0034R & $0.07(-0.05-0.20)$ & & 0.268 & $0.48(-0.07-1.10)$ & + & 0.093 \\
\hline AT0044A & $0.13(0.01-0.26)$ & $*$ & 0.032 & $1.23(0.29-2.26)$ & $*$ & 0.013 \\
\hline AT0052A & $-0.03(-0.11-0.08)$ & & 0.568 & $-0.11(-0.56-0.44)$ & & 0.693 \\
\hline AT0054A & $0.11(-0.04-0.25)$ & & 0.102 & $0.95(-0.04-2.15)$ & * & 0.033 \\
\hline AT0058A & $0.11(-0.03-0.24)$ & & 0.125 & $0.48(-0.03-1.03)$ & + & 0.065 \\
\hline AT0064A & $0.07(-0.06-0.18)$ & & 0.314 & $0.20(-0.30-0.82)$ & & 0.454 \\
\hline AT0069A & $0.14(0.02-0.26)$ & $*$ & 0.018 & $1.05(0.10-2.04)$ & $*$ & 0.024 \\
\hline AT0073A & $0.11(-0.04-0.23)$ & + & 0.082 & $0.87(-0.10-1.82)$ & + & 0.058 \\
\hline AT0079A & $0.07(-0.05-0.20)$ & & 0.268 & $0.48(-0.07-1.10)$ & + & 0.093 \\
\hline AT0080A & $0.03(-0.09-0.15)$ & & 0.534 & $0.45(-0.39-1.21)$ & & 0.226 \\
\hline AT0086A & $0.07(-0.04-0.17)$ & & 0.156 & $0.66(0.01-1.21)$ & $*$ & 0.028 \\
\hline AT0089A & $0.10(-0.03-0.22)$ & & 0.169 & $0.23(-0.32-0.84)$ & & 0.465 \\
\hline AT0094A & $-0.03(-0.11-0.08)$ & & 0.568 & $-0.11(-0.56-0.44)$ & & 0.693 \\
\hline AT0095A & $0.13(0.00-0.26)$ & + & 0.057 & $1.51(0.32-2.96)$ & * & 0.017 \\
\hline AT0096A & $0.03(-0.11-0.16)$ & & 0.624 & $0.22(-0.62-1.29)$ & & 0.596 \\
\hline AT0101A & $0.29(0.14-0.41)$ & $* * *$ & $<0.001$ & $1.70(0.90-2.58)$ & $* * *$ & $<0.001$ \\
\hline AT0102A & $0.13(0.00-0.26)$ & + & 0.057 & $1.51(0.32-2.96)$ & $*$ & 0.017 \\
\hline AT0103A & $0.13(0.00-0.23)$ & $*$ & 0.032 & $1.01(-0.10-1.98)$ & + & 0.060 \\
\hline AT0105A & $0.14(0.01-0.27)$ & $*$ & 0.023 & $1.08(0.15-2.01)$ & $*$ & 0.015 \\
\hline AT0108A & $0.04(-0.06-0.15)$ & & 0.417 & $0.33(-0.33-1.12)$ & & 0.352 \\
\hline AT0111A & $0.14(0.01-0.27)$ & $*$ & 0.023 & $1.08(0.15-2.01)$ & $*$ & 0.015 \\
\hline AT0115A & $0.07(-0.05-0.21)$ & & 0.255 & $0.18(-0.37-0.68)$ & & 0.514 \\
\hline AT0121A & $0.08(-0.04-0.20)$ & & 0.196 & $0.19(-0.37-0.75)$ & & 0.546 \\
\hline AT0122A & $0.17(0.04-0.29)$ & $*$ & 0.011 & $0.66(0.06-1.32)$ & $*$ & 0.031 \\
\hline AT0124A & $-0.01(-0.12-0.10)$ & & 0.892 & $-0.05(-0.56-0.42)$ & & 0.817 \\
\hline AT0128A & $0.05(-0.05-0.13)$ & & 0.288 & $0.40(-0.12-0.90)$ & & 0.150 \\
\hline AT0134A & $0.06(-0.07-0.18)$ & & 0.286 & $0.47(-0.26-1.22)$ & & 0.198 \\
\hline AT0141A & $0.07(-0.05-0.20)$ & & 0.268 & $0.48(-0.07-1.10)$ & + & 0.093 \\
\hline AT0143A & $0.05(-0.05-0.13)$ & & 0.288 & $0.40(-0.12-0.90)$ & & 0.150 \\
\hline AT0146A & $0.04(-0.07-0.17)$ & & 0.508 & $0.21(-0.34-0.85)$ & & 0.511 \\
\hline AT0149A & $0.13(0.01-0.26)$ & $*$ & 0.032 & $1.23(0.29-2.26)$ & $*$ & 0.013 \\
\hline AT0153A & $0.12(-0.02-0.22)$ & $*$ & 0.043 & $0.93(0.07-1.77)$ & $*$ & 0.030 \\
\hline AT0154A & $0.10(-0.01-0.21)$ & + & 0.094 & $0.64(-0.11-1.53)$ & + & 0.098 \\
\hline AT0162A & $0.07(-0.05-0.21)$ & & 0.255 & $0.18(-0.37-0.68)$ & & 0.514 \\
\hline AT0164A & $0.04(-0.06-0.15)$ & & 0.417 & $0.33(-0.33-1.12)$ & & 0.352 \\
\hline AT0166A & $0.10(-0.04-0.22)$ & & 0.125 & $0.53(-0.22-1.15)$ & & 0.129 \\
\hline AT0167A & $0.04(-0.06-0.15)$ & & 0.417 & $0.33(-0.33-1.12)$ & & 0.352 \\
\hline AT0175A & $0.07(-0.05-0.18)$ & & 0.236 & $0.52(-0.44-1.44)$ & & 0.222 \\
\hline AT0176A & $0.03(-0.09-0.15)$ & & 0.534 & $0.45(-0.39-1.21)$ & & 0.226 \\
\hline
\end{tabular}


Table B2: continued. Quantification of annual CHIMERE model trends (ppbv/yr and $\% / \mathrm{yr}$ ) in $\mathrm{O}_{3} 5^{\text {th }}$ percentiles 1996-2005. 95\% confidence interval given in brackets. Sig. $=$ significance ${ }^{* * *}$ significant at $99.9 \%$ level, ${ }^{* *}$ significant at $99.0 \%$ level, ${ }^{*}$ significant at $95.0 \%$ level, + significant at $90.0 \%$ level. ${ }^{\ddagger} 2 \sigma$ error. ${ }^{\diamond}$ significant trends only

\begin{tabular}{|c|c|c|c|c|c|c|}
\hline ID & Trend (ppbv/yr) & Sig. & $\mathrm{P}$-value & Trend (\%/yr) & Sig. & $\mathrm{P}$-value \\
\hline AT0180A & $0.07(-0.05-0.18)$ & & 0.295 & $0.39(-0.22-1.02)$ & & 0.209 \\
\hline BE0032R & $0.09(-0.09-0.24)$ & & 0.329 & $0.49(-1.00-2.08)$ & & 0.534 \\
\hline BE0033R & $0.06(-0.14-0.25)$ & & 0.522 & $0.31(-1.63-2.89)$ & & 0.727 \\
\hline BE0035R & $0.14(-0.01-0.30)$ & + & 0.059 & $1.31(-0.45-3.54)$ & & 0.148 \\
\hline BE0211A & $0.07(-0.02-0.19)$ & & 0.150 & $2.12(-1.08-7.09)$ & & 0.196 \\
\hline BE0238A & $0.09(-0.10-0.26)$ & & 0.251 & $0.61(-0.43-1.62)$ & & 0.194 \\
\hline BE0294A & $0.14(-0.01-0.30)$ & + & 0.059 & $1.31(-0.45-3.54)$ & & 0.148 \\
\hline BE0298A & $0.06(-0.01-0.17)$ & & 0.147 & $1.65(-2.73-6.41)$ & & 0.371 \\
\hline BE0302A & $0.14(-0.01-0.28)$ & + & 0.064 & $1.03(0.08-2.20)$ & $*$ & 0.028 \\
\hline BE0304A & $0.12(-0.07-0.29)$ & & 0.158 & $0.96(-0.29-2.42)$ & & 0.145 \\
\hline BE0311A & $0.10(-0.06-0.27)$ & & 0.190 & $0.83(-0.14-1.81)$ & & 0.106 \\
\hline BE0345A & $0.06(-0.01-0.17)$ & & 0.147 & $1.65(-2.73-6.41)$ & & 0.371 \\
\hline CH0001R & $0.03(-0.13-0.19)$ & & 0.734 & $0.02(-0.59-0.72)$ & & 0.921 \\
\hline $\mathrm{CH} 0002 \mathrm{R}$ & $0.03(-0.11-0.15)$ & & 0.723 & $0.10(-0.62-0.85)$ & & 0.751 \\
\hline CH0003R & $0.13(0.00-0.24)$ & $*$ & 0.039 & $0.87(0.04-1.80)$ & $*$ & 0.029 \\
\hline CH0004R & $0.03(-0.11-0.15)$ & & 0.723 & $0.10(-0.62-0.85)$ & & 0.751 \\
\hline CH0005R & $0.18(0.05-0.30)$ & $* *$ & 0.005 & $0.96(0.31-1.72)$ & $* *$ & 0.003 \\
\hline CH0019A & $0.10(-0.02-0.21)$ & + & 0.081 & $0.60(-0.10-1.25)$ & + & 0.083 \\
\hline CH0024A & $-0.09(-0.20-0.05)$ & & 0.198 & $-0.27(-0.76-0.32)$ & & 0.293 \\
\hline CH0033A & $0.24(0.11-0.36)$ & $* * *$ & $<0.001$ & $2.43(0.99-4.71)$ & $* * *$ & $<0.001$ \\
\hline $\mathrm{CMN}$ & $0.11(-0.05-0.30)$ & & 0.165 & $0.56(-0.24-1.46)$ & & 0.142 \\
\hline CZ0001R & $0.37(0.19-0.53)$ & $* * *$ & $<0.001$ & $2.53(1.26-3.70)$ & $* * *$ & $<0.001$ \\
\hline CZ0003R & $0.36(0.24-0.48)$ & $* * *$ & $<0.001$ & $2.35(1.50-3.17)$ & $* * *$ & $<0.001$ \\
\hline CZ0017A & $0.27(0.16-0.39)$ & $* * *$ & $<0.001$ & $1.83(1.15-2.69)$ & $* * *$ & $<0.001$ \\
\hline CZ0030A & $0.23(0.08-0.37)$ & $* *$ & 0.003 & $2.04(0.99-3.33)$ & $* * *$ & $<0.001$ \\
\hline CZ0041A & $0.56(0.40-0.72)$ & $* * *$ & $<0.001$ & $4.81(3.38-6.47)$ & $* * *$ & $<0.001$ \\
\hline CZ0045A & $0.31(0.17-0.43)$ & $* * *$ & $<0.001$ & $1.97(1.07-2.89)$ & $* * *$ & $<0.001$ \\
\hline CZ0049A & $0.20(0.07-0.32)$ & $* *$ & 0.002 & $1.27(0.42-2.36)$ & $* *$ & 0.002 \\
\hline CZ0051A & $0.35(0.18-0.53)$ & $* * *$ & $<0.001$ & $2.72(1.45-3.96)$ & $* * *$ & $<0.001$ \\
\hline CZ0055A & $0.34(0.21-0.46)$ & $* * *$ & $<0.001$ & $2.40(1.49-3.30)$ & $* * *$ & $<0.001$ \\
\hline CZ0057A & $0.11(-0.04-0.23)$ & + & 0.082 & $0.87(-0.10-1.82)$ & + & 0.058 \\
\hline CZ0062A & $0.32(0.15-0.46)$ & $* * *$ & $<0.001$ & $2.95(1.92-4.09)$ & $* * *$ & $<0.001$ \\
\hline DE0003R & $0.15(0.03-0.27)$ & $*$ & 0.016 & $1.71(0.57-3.34)$ & $* *$ & 0.007 \\
\hline DE0007R & $0.09(-0.06-0.27)$ & & 0.253 & $0.92(-0.58-2.47)$ & & 0.242 \\
\hline DE0008R & $0.05(-0.08-0.17)$ & & 0.496 & $0.32(-0.48-1.34)$ & & 0.449 \\
\hline DE0009R & $0.09(-0.12-0.30)$ & & 0.460 & $0.63(-0.59-1.63)$ & & 0.251 \\
\hline DE0035R & $0.21(0.04-0.36)$ & $* *$ & 0.010 & $1.79(0.56-3.33)$ & $* *$ & 0.003 \\
\hline DE0422A & $0.28(0.15-0.44)$ & $* * *$ & $<0.001$ & $3.40(1.01-6.66)$ & $* *$ & 0.006 \\
\hline DE0510A & $0.10(-0.05-0.26)$ & & 0.162 & $1.04(-0.53-3.29)$ & & 0.172 \\
\hline DE0514A & $0.14(0.02-0.26)$ & $*$ & 0.018 & $1.05(0.10-2.04)$ & $*$ & 0.024 \\
\hline DE0556A & $0.32(0.15-0.46)$ & $* * *$ & $<0.001$ & $2.95(1.92-4.09)$ & $* * *$ & $<0.001$ \\
\hline DE0649A & $0.18(0.05-0.33)$ & $*$ & 0.014 & $1.20(0.28-2.36)$ & $*$ & 0.016 \\
\hline
\end{tabular}


Table B2: continued. Quantification of annual CHIMERE model trends (ppbv/yr and $\% / \mathrm{yr}$ ) in $\mathrm{O}_{3} 5^{\text {th }}$ percentiles 1996-2005. 95\% confidence interval given in brackets. Sig. $=$ significance ${ }^{* * *}$ significant at $99.9 \%$ level, ${ }^{* *}$ significant at $99.0 \%$ level, ${ }^{*}$ significant at $95.0 \%$ level, + significant at $90.0 \%$ level. ${ }^{\ddagger} 2 \sigma$ error. ${ }^{\diamond}$ significant trends only

\begin{tabular}{|c|c|c|c|c|c|c|}
\hline ID & Trend (ppbv/yr) & Sig. & P-value & Trend (\%/yr) & Sig. & $\mathrm{P}$-value \\
\hline$\overline{\mathrm{DE}} 0651 \mathrm{~A}$ & $0.20(0.08-0.34)$ & $* *$ & 0.008 & $1.77(0.77-3.07)$ & $* *$ & 0.002 \\
\hline DE0674A & $0.10(-0.06-0.27)$ & & 0.184 & $0.97(-0.39-2.73)$ & & 0.139 \\
\hline DE0679A & $0.17(0.04-0.29)$ & $*$ & 0.013 & $1.20(0.14-2.40)$ & $*$ & 0.016 \\
\hline DE0680A & $0.21(0.06-0.36)$ & $* *$ & 0.008 & $1.93(0.13-3.71)$ & $*$ & 0.026 \\
\hline DE0684A & $0.15(0.03-0.27)$ & $*$ & 0.016 & $1.71(0.57-3.34)$ & $* *$ & 0.007 \\
\hline DE0685A & $0.16(0.01-0.34)$ & + & 0.061 & $1.46(-0.13-3.36)$ & + & 0.075 \\
\hline DE0686A & $0.12(-0.02-0.27)$ & & 0.122 & $0.89(-0.19-2.21)$ & & 0.108 \\
\hline DE0687A & $0.10(-0.06-0.27)$ & & 0.184 & $0.97(-0.39-2.73)$ & & 0.139 \\
\hline DE0688A & $0.25(0.08-0.42)$ & $* *$ & 0.003 & $2.06(0.63-3.82)$ & $* *$ & 0.005 \\
\hline DE0699A & $0.15(0.04-0.28)$ & $* *$ & 0.007 & $2.80(1.06-5.16)$ & $* *$ & 0.001 \\
\hline DE0719A & $0.20(0.09-0.34)$ & $* *$ & 0.002 & $1.51(0.43-2.94)$ & $* *$ & 0.006 \\
\hline DE0732A & $0.22(0.07-0.38)$ & $* *$ & 0.004 & $1.96(0.49-3.60)$ & $* *$ & 0.008 \\
\hline DE0735A & $0.25(0.08-0.42)$ & $* *$ & 0.003 & $2.06(0.63-3.82)$ & $* *$ & 0.005 \\
\hline DE0737A & $0.21(0.07-0.37)$ & $* *$ & 0.009 & $3.15(1.02-6.06)$ & $* *$ & 0.004 \\
\hline DE0738A & $0.09(-0.05-0.21)$ & & 0.224 & $0.66(-0.10-1.56)$ & & 0.130 \\
\hline DE0739A & $0.19(0.08-0.31)$ & $* *$ & 0.006 & $2.18(0.64-4.45)$ & $* *$ & 0.008 \\
\hline DE0754A & $0.15(0.00-0.32)$ & + & 0.075 & $2.24(0.51-4.45)$ & $*$ & 0.016 \\
\hline DE0844A & $0.14(-0.06-0.28)$ & & 0.130 & $2.16(-0.10-4.34)$ & + & 0.064 \\
\hline DE0874A & $0.27(0.13-0.43)$ & $* * *$ & $<0.001$ & $3.40(1.00-6.71)$ & $* *$ & 0.006 \\
\hline DE0907A & $0.28(0.14-0.43)$ & $* * *$ & 0.001 & $2.88(1.19-4.79)$ & $* * *$ & $<0.001$ \\
\hline DE0960A & $0.07(-0.08-0.25)$ & & 0.367 & $0.55(-0.58-1.68)$ & & 0.348 \\
\hline DE0996A & $0.18(0.02-0.33)$ & $*$ & 0.036 & $1.83(0.32-3.23)$ & $*$ & 0.013 \\
\hline EE0011R & $0.17(0.03-0.31)$ & $*$ & 0.011 & $0.56(0.07-1.05)$ & $*$ & 0.029 \\
\hline ES0007R & $-0.04(-0.16-0.10)$ & & 0.485 & $-0.19(-0.65-0.33)$ & & 0.409 \\
\hline ES1222A & $0.10(-0.13-0.32)$ & & 0.316 & $0.41(-0.45-1.26)$ & & 0.336 \\
\hline ES1400A & $0.11(-0.01-0.20)$ & + & 0.059 & $0.42(-0.07-0.80)$ & + & 0.077 \\
\hline ES1435A & $0.07(-0.04-0.18)$ & & 0.194 & $0.24(-0.17-0.60)$ & & 0.242 \\
\hline ES1437A & $0.03(-0.11-0.15)$ & & 0.680 & $0.15(-0.42-0.66)$ & & 0.531 \\
\hline ES1441A & $0.03(-0.11-0.15)$ & & 0.680 & $0.15(-0.42-0.66)$ & & 0.531 \\
\hline FR08 & $0.12(-0.04-0.28)$ & & 0.171 & $0.67(-0.45-2.09)$ & & 0.284 \\
\hline GB0002R & $0.07(-0.11-0.25)$ & & 0.451 & $0.19(-0.74-1.04)$ & & 0.670 \\
\hline GB0006R & $0.05(-0.11-0.20)$ & & 0.568 & $0.10(-0.40-0.64)$ & & 0.730 \\
\hline GB0013R & $0.15(0.00-0.37)$ & + & 0.067 & $0.80(0.01-1.81)$ & $*$ & 0.048 \\
\hline GB0014R & $0.35(0.16-0.56)$ & $* *$ & 0.002 & $2.04(0.80-3.45)$ & $* *$ & 0.002 \\
\hline GB0015R & $-0.07(-0.22-0.06)$ & & 0.310 & $-0.22(-0.78-0.28)$ & & 0.406 \\
\hline GB0031R & $0.31(0.11-0.54)$ & $* *$ & 0.003 & $1.68(0.71-3.12)$ & $* * *$ & 0.001 \\
\hline GB0033R & $0.18(0.00-0.38)$ & $*$ & 0.033 & $0.90(-0.08-1.84)$ & $*$ & 0.030 \\
\hline GB0036R & $0.50(0.29-0.74)$ & $* * *$ & $<0.001$ & $4.17(1.93-7.07)$ & $* * *$ & $<0.001$ \\
\hline GB0037R & $0.45(0.24-0.65)$ & $* * *$ & $<0.001$ & $5.57(1.71-10.35)$ & $* *$ & 0.003 \\
\hline GB0038R & $0.46(0.27-0.69)$ & $* * *$ & $<0.001$ & $4.20(1.38-7.79)$ & $* *$ & 0.010 \\
\hline GB0039R & $0.08(-0.14-0.32)$ & & 0.568 & $0.20(-1.21-1.80)$ & & 0.814 \\
\hline GB0044R & $0.30(-0.01-0.59)$ & $*$ & 0.046 & $1.69(0.07-3.68)$ & $*$ & 0.036 \\
\hline
\end{tabular}


Table B2: continued. Quantification of annual CHIMERE model trends (ppbv/yr and $\% / \mathrm{yr}$ ) in $\mathrm{O}_{3} 5^{\text {th }}$ percentiles 1996-2005. 95\% confidence interval given in brackets. Sig. $=$ significance $* * *$ significant at $99.9 \%$ level, $* *$ significant at $99.0 \%$ level, ${ }^{*}$ significant at $95.0 \%$ level, + significant at $90.0 \%$ level. ${ }^{\ddagger} 2 \sigma$ error. ${ }^{\diamond}$ significant trends only

\begin{tabular}{|c|c|c|c|c|c|c|}
\hline ID & Trend (ppbv/yr) & Sig. & P-value & Trend (\%/yr) & Sig. & P-value \\
\hline GB0045R & $0.38(0.17-0.62)$ & $* * *$ & 0.001 & $4.32(1.28-8.63)$ & ** & 0.003 \\
\hline GB0617A & $0.33(0.18-0.51)$ & $* * *$ & $<0.001$ & $5.77(2.30-10.38)$ & $* *$ & 0.001 \\
\hline HPB & $0.20(0.06-0.33)$ & $* *$ & 0.004 & $1.75(0.62-3.18)$ & $* *$ & 0.002 \\
\hline HU0002R & $-0.09(-0.21-0.02)$ & + & 0.070 & $-0.47(-1.42-0.46)$ & & 0.204 \\
\hline IE31 & $0.02(-0.09-0.15)$ & & 0.690 & $0.11(-0.31-0.51)$ & & 0.583 \\
\hline IT04 & $0.11(-0.03-0.26)$ & & 0.143 & $0.98(-0.01-2.24)$ & + & 0.050 \\
\hline LT0015R & $0.13(-0.05-0.31)$ & & 0.160 & $0.66(-0.13-1.42)$ & + & 0.086 \\
\hline LV0010R & $0.15(-0.05-0.33)$ & & 0.105 & $0.71(-0.20-1.52)$ & + & 0.087 \\
\hline NL0007R & $0.13(0.02-0.26)$ & $*$ & 0.019 & $1.64(-1.08-6.23)$ & & 0.217 \\
\hline NL0009R & $0.19(-0.08-0.42)$ & & 0.152 & $1.33(-0.12-3.07)$ & + & 0.092 \\
\hline NL0010R & $0.09(0.00-0.20)$ & + & 0.056 & $2.50(-1.39-8.10)$ & & 0.153 \\
\hline NL0196A & $0.06(-0.04-0.18)$ & & 0.236 & $1.23(-2.12-5.62)$ & & 0.462 \\
\hline NL0198A & $0.14(-0.01-0.32)$ & & 0.108 & $0.98(-1.78-4.22)$ & & 0.381 \\
\hline NL0202A & $0.09(-0.02-0.21)$ & + & 0.067 & $1.90(-0.72-5.47)$ & & 0.150 \\
\hline NL0205A & $0.16(0.00-0.33)$ & $*$ & 0.044 & $1.74(-0.54-4.72)$ & & 0.132 \\
\hline NL0207A & $0.07(-0.16-0.31)$ & & 0.534 & $1.00(-0.50-2.90)$ & & 0.207 \\
\hline NL0209A & $0.05(-0.03-0.15)$ & & 0.202 & $1.70(-3.87-6.42)$ & & 0.482 \\
\hline NL0220A & $0.06(-0.14-0.25)$ & & 0.522 & $0.31(-1.63-2.89)$ & & 0.727 \\
\hline NL0223A & $0.06(-0.03-0.19)$ & & 0.162 & $1.73(-2.84-7.38)$ & & 0.422 \\
\hline NL0226A & $0.01(-0.09-0.13)$ & & 0.650 & $1.36(-3.92-6.76)$ & & 0.574 \\
\hline NL0227A & $0.01(-0.23-0.29)$ & & 0.939 & $0.71(-0.80-2.62)$ & & 0.374 \\
\hline NL0228A & $0.10(-0.09-0.31)$ & & 0.246 & $0.98(-0.96-3.75)$ & & 0.320 \\
\hline NL0229A & $0.05(-0.03-0.15)$ & & 0.202 & $1.70(-3.87-6.42)$ & & 0.482 \\
\hline NL0231A & $0.14(-0.02-0.32)$ & + & 0.078 & $1.71(-0.36-4.39)$ & & 0.123 \\
\hline NL0232A & $0.06(-0.02-0.16)$ & & 0.165 & $1.04(-3.57-5.72)$ & & 0.580 \\
\hline NL0250A & $-0.05(-0.27-0.17)$ & & 0.690 & $-1.16(-3.37-1.22)$ & & 0.293 \\
\hline NO01 & $0.11(-0.06-0.32)$ & & 0.251 & $0.37(-0.26-1.19)$ & & 0.282 \\
\hline PL03 & $0.33(0.17-0.46)$ & $* * *$ & $<0.001$ & $2.66(1.61-3.82)$ & $* * *$ & $<0.001$ \\
\hline PT0004R & $-0.13(-0.29-0.04)$ & & 0.118 & $-0.37(-0.90-0.17)$ & & 0.140 \\
\hline PUY & $0.11(-0.03-0.22)$ & & 0.117 & $0.42(-0.15-0.96)$ & & 0.136 \\
\hline SI0008R & $-0.08(-0.21-0.06)$ & & 0.206 & $-0.27(-0.93-0.35)$ & & 0.303 \\
\hline ZUG & $0.10(-0.03-0.22)$ & & 0.169 & $0.23(-0.32-0.84)$ & & 0.465 \\
\hline $\begin{array}{c}\text { European } \\
\text { average }^{\ddagger}\end{array}$ & $0.14 \pm 0.01 \mathrm{ppbv} / \mathrm{yr}$ & & & $1.19 \pm 0.15 \% / \mathrm{yr}$ & & \\
\hline Range $^{\diamond}$ & -0.09 to $0.56 \mathrm{ppbv} / \mathrm{yr}$ & & & -6.32 to $20.02 \% / y r$ & & \\
\hline
\end{tabular}


Table B3: Quantification of annual CHIMERE model trends (ppbv/yr and \%/yr) in $\mathrm{O}_{3} 95^{\text {th }}$ percentiles 1996-2005. 95\% confidence interval given in brackets. Sig. = significance ${ }^{* * *}$ significant at $99.9 \%$ level, ${ }^{* *}$ significant at $99.0 \%$ level, ${ }^{*}$ significant at $95.0 \%$ level, + significant at $90.0 \%$ level. ${ }^{\ddagger} 2 \sigma$ error. ${ }^{\diamond}$ significant trends only

\begin{tabular}{|c|c|c|c|c|c|c|}
\hline ID & Trend (ppbv/yr) & Sig. & P-value & Trend $(\% / y r)$ & Sig. & $\mathrm{P}$-value \\
\hline AT0002R & $-0.06(-0.25-0.17)$ & & 0.574 & $-0.13(-0.57-0.32)$ & & 0.558 \\
\hline AT0004R & $-0.05(-0.24-0.13)$ & & 0.583 & $-0.06(-0.44-0.27)$ & & 0.758 \\
\hline AT0005R & $-0.14(-0.35-0.11)$ & & 0.209 & $-0.17(-0.60-0.30)$ & & 0.396 \\
\hline AT0034R & $-0.10(-0.27-0.07)$ & & 0.261 & $-0.18(-0.52-0.18)$ & & 0.282 \\
\hline AT0044A & $-0.02(-0.24-0.22)$ & & 0.867 & $-0.04(-0.49-0.45)$ & & 0.856 \\
\hline AT0052A & $-0.02(-0.21-0.18)$ & & 0.772 & $-0.01(-0.45-0.39)$ & & 0.967 \\
\hline AT0054A & $0.01(-0.18-0.20)$ & & 0.942 & $0.06(-0.30-0.52)$ & & 0.775 \\
\hline AT0058A & $-0.15(-0.33-0.00)$ & $*$ & 0.049 & $-0.25(-0.58-0.07)$ & & 0.120 \\
\hline AT0064A & $-0.15(-0.32-0.05)$ & & 0.113 & $-0.24(-0.61-0.18)$ & & 0.209 \\
\hline AT0069A & $-0.01(-0.23-0.21)$ & & 0.921 & $0.03(-0.45-0.52)$ & & 0.877 \\
\hline AT0073A & $-0.04(-0.20-0.13)$ & & 0.615 & $-0.06(-0.40-0.34)$ & & 0.775 \\
\hline AT0079A & $-0.10(-0.27-0.07)$ & & 0.261 & $-0.18(-0.52-0.18)$ & & 0.282 \\
\hline AT0080A & $0.01(-0.20-0.24)$ & & 0.935 & $0.04(-0.38-0.52)$ & & 0.828 \\
\hline AT0086A & $-0.04(-0.26-0.18)$ & & 0.683 & $-0.02(-0.46-0.49)$ & & 0.939 \\
\hline AT0089A & $-0.16(-0.33-0.03)$ & + & 0.068 & $-0.24(-0.59-0.16)$ & & 0.199 \\
\hline AT0094A & $-0.02(-0.21-0.18)$ & & 0.772 & $-0.01(-0.45-0.39)$ & & 0.967 \\
\hline AT0095A & $-0.04(-0.27-0.17)$ & & 0.666 & $-0.10(-0.56-0.37)$ & & 0.676 \\
\hline AT0096A & $-0.05(-0.27-0.20)$ & & 0.673 & $-0.01(-0.52-0.45)$ & & 0.978 \\
\hline AT0101A & $-0.03(-0.22-0.17)$ & & 0.737 & $-0.07(-0.45-0.35)$ & & 0.741 \\
\hline AT0102A & $-0.04(-0.27-0.17)$ & & 0.666 & $-0.10(-0.56-0.37)$ & & 0.676 \\
\hline AT0103A & $-0.04(-0.26-0.15)$ & & 0.686 & $-0.08(-0.52-0.35)$ & & 0.803 \\
\hline AT0105A & $-0.05(-0.28-0.19)$ & & 0.676 & $-0.04(-0.51-0.44)$ & & 0.831 \\
\hline AT0108A & $-0.01(-0.21-0.20)$ & & 0.949 & $0.00(-0.41-0.45)$ & & 0.989 \\
\hline AT0111A & $-0.05(-0.28-0.19)$ & & 0.676 & $-0.04(-0.51-0.44)$ & & 0.831 \\
\hline AT0115A & $-0.04(-0.20-0.15)$ & & 0.700 & $-0.03(-0.39-0.35)$ & & 0.852 \\
\hline AT0121A & $-0.18(-0.36-0.01)$ & $*$ & 0.045 & $-0.29(-0.68-0.12)$ & & 0.123 \\
\hline AT0122A & $-0.08(-0.26-0.11)$ & & 0.367 & $-0.15(-0.52-0.22)$ & & 0.371 \\
\hline AT0124A & $-0.14(-0.31-0.06)$ & & 0.132 & $-0.25(-0.60-0.13)$ & & 0.174 \\
\hline AT0128A & $-0.07(-0.27-0.13)$ & & 0.443 & $-0.14(-0.57-0.24)$ & & 0.528 \\
\hline AT0134A & $-0.04(-0.28-0.21)$ & & 0.765 & $-0.04(-0.50-0.51)$ & & 0.917 \\
\hline AT0141A & $-0.10(-0.27-0.07)$ & & 0.261 & $-0.18(-0.52-0.18)$ & & 0.282 \\
\hline AT0143A & $-0.07(-0.27-0.13)$ & & 0.443 & $-0.14(-0.57-0.24)$ & & 0.528 \\
\hline AT0146A & $-0.03(-0.18-0.13)$ & & 0.683 & $-0.05(-0.37-0.32)$ & & 0.782 \\
\hline AT0149A & $-0.02(-0.24-0.22)$ & & 0.867 & $-0.04(-0.49-0.45)$ & & 0.856 \\
\hline AT0153A & $-0.09(-0.29-0.12)$ & & 0.367 & $-0.17(-0.53-0.27)$ & & 0.401 \\
\hline AT0154A & $-0.05(-0.24-0.14)$ & & 0.640 & $-0.07(-0.48-0.36)$ & & 0.761 \\
\hline AT0162A & $-0.04(-0.20-0.15)$ & & 0.700 & $-0.03(-0.39-0.35)$ & & 0.852 \\
\hline AT0164A & $-0.01(-0.21-0.20)$ & & 0.949 & $0.00(-0.41-0.45)$ & & 0.989 \\
\hline AT0166A & $-0.05(-0.24-0.13)$ & & 0.583 & $-0.06(-0.44-0.27)$ & & 0.758 \\
\hline AT0167A & $-0.01(-0.21-0.20)$ & & 0.949 & $0.00(-0.41-0.45)$ & & 0.989 \\
\hline AT0175A & $-0.08(-0.30-0.17)$ & & 0.522 & $-0.19(-0.64-0.30)$ & & 0.449 \\
\hline AT0176A & $0.01(-0.20-0.24)$ & & 0.935 & $0.04(-0.38-0.52)$ & & 0.828 \\
\hline
\end{tabular}


Table B3: Quantification of annual CHIMERE model trends (ppbv/yr and \%/yr) in $\mathrm{O}_{3} 95^{\text {th }}$ percentiles 1996-2005. 95\% confidence interval given in brackets. Sig. = significance ${ }^{* * *}$ significant at $99.9 \%$ level, ${ }^{* *}$ significant at $99.0 \%$ level, ${ }^{*}$ significant at $95.0 \%$ level, + significant at $90.0 \%$ level. ${ }^{\ddagger} 2 \sigma$ error. ${ }^{\diamond}$ significant trends only

\begin{tabular}{|c|c|c|c|c|c|c|}
\hline ID & Trend (ppbv/yr) & Sig. & P-value & Trend (\%/yr) & Sig. & P-value \\
\hline$\overline{\text { AT0180A }}$ & $-0.02(-0.21-0.15)$ & & 0.799 & $-0.06(-0.42-0.30)$ & & 0.727 \\
\hline BE0032R & $0.03(-0.16-0.28)$ & & 0.785 & $0.07(-0.37-0.58)$ & & 0.772 \\
\hline BE0033R & $-0.03(-0.20-0.12)$ & & 0.586 & $-0.08(-0.44-0.29)$ & & 0.602 \\
\hline BE0035R & $0.05(-0.12-0.26)$ & & 0.627 & $0.11(-0.27-0.60)$ & & 0.596 \\
\hline BE0211A & $0.03(-0.17-0.24)$ & & 0.720 & $0.14(-0.32-0.66)$ & & 0.543 \\
\hline BE0238A & $0.03(-0.14-0.21)$ & & 0.710 & $0.06(-0.31-0.44)$ & & 0.730 \\
\hline BE0294A & $0.05(-0.12-0.26)$ & & 0.627 & $0.11(-0.27-0.60)$ & & 0.596 \\
\hline BE0298A & $0.10(-0.12-0.32)$ & & 0.371 & $0.18(-0.37-0.76)$ & & 0.537 \\
\hline BE0302A & $0.00(-0.19-0.25)$ & & 0.971 & $0.02(-0.42-0.51)$ & & 0.942 \\
\hline BE0304A & $0.06(-0.09-0.22)$ & & 0.449 & $0.15(-0.21-0.56)$ & & 0.488 \\
\hline BE0311A & $0.04(-0.16-0.23)$ & & 0.663 & $0.10(-0.36-0.50)$ & & 0.624 \\
\hline BE0345A & $0.10(-0.12-0.32)$ & & 0.371 & $0.18(-0.37-0.76)$ & & 0.537 \\
\hline CH0001R & $-0.07(-0.29-0.17)$ & & 0.534 & $-0.13(-0.51-0.32)$ & & 0.499 \\
\hline CH0002R & $0.01(-0.23-0.29)$ & & 0.953 & $-0.03(-0.46-0.50)$ & & 0.903 \\
\hline CH0003R & $-0.22(-0.45-0.06)$ & + & 0.091 & $-0.38(-0.84-0.23)$ & & 0.188 \\
\hline CH0004R & $0.01(-0.23-0.29)$ & & 0.953 & $-0.03(-0.46-0.50)$ & & 0.903 \\
\hline CH0005R & $-0.15(-0.36-0.13)$ & & 0.233 & $-0.20(-0.61-0.30)$ & & 0.357 \\
\hline CH0019A & $-0.24(-0.45-0.02)$ & + & 0.060 & $-0.35(-0.79-0.13)$ & & 0.122 \\
\hline $\mathrm{CH} 0024 \mathrm{~A}$ & $-0.06(-0.24-0.13)$ & & 0.468 & $-0.12(-0.44-0.24)$ & & 0.508 \\
\hline CH0033A & $-0.29(-0.61-0.06)$ & + & 0.064 & $-0.33(-0.86-0.26)$ & & 0.222 \\
\hline CMN & $-0.46(-0.72--0.23)$ & $* * *$ & $<0.001$ & $-0.73(-1.13--0.30)$ & $* *$ & 0.002 \\
\hline CZ0001R & $-0.03(-0.25-0.18)$ & & 0.792 & $-0.10(-0.57-0.38)$ & & 0.737 \\
\hline CZ0003R & $-0.05(-0.24-0.18)$ & & 0.640 & $-0.12(-0.58-0.38)$ & & 0.586 \\
\hline CZ0017A & $-0.03(-0.24-0.17)$ & & 0.785 & $-0.17(-0.65-0.31)$ & & 0.514 \\
\hline CZ0030A & $-0.02(-0.24-0.17)$ & & 0.838 & $-0.08(-0.53-0.35)$ & & 0.707 \\
\hline CZ0041A & $-0.06(-0.29-0.17)$ & & 0.596 & $-0.18(-0.68-0.35)$ & & 0.474 \\
\hline CZ0045A & $-0.02(-0.24-0.21)$ & & 0.852 & $-0.02(-0.47-0.42)$ & & 0.917 \\
\hline CZ0049A & $-0.05(-0.24-0.16)$ & & 0.634 & $-0.06(-0.44-0.41)$ & & 0.782 \\
\hline CZ0051A & $-0.03(-0.25-0.17)$ & & 0.696 & $-0.09(-0.57-0.35)$ & & 0.680 \\
\hline CZ0055A & $-0.08(-0.28-0.12)$ & & 0.465 & $-0.15(-0.62-0.30)$ & & 0.496 \\
\hline CZ0057A & $-0.04(-0.20-0.13)$ & & 0.615 & $-0.06(-0.40-0.34)$ & & 0.775 \\
\hline CZ0062A & $0.03(-0.20-0.28)$ & & 0.789 & $0.04(-0.48-0.57)$ & & 0.856 \\
\hline DE0003R & $0.03(-0.24-0.35)$ & & 0.775 & $-0.06(-0.59-0.47)$ & & 0.772 \\
\hline DE0007R & $-0.03(-0.24-0.17)$ & & 0.789 & $-0.09(-0.55-0.39)$ & & 0.734 \\
\hline DE0008R & $0.02(-0.21-0.25)$ & & 0.881 & $-0.05(-0.57-0.51)$ & & 0.845 \\
\hline DE0009R & $-0.09(-0.26-0.04)$ & & 0.219 & $-0.17(-0.51-0.13)$ & & 0.338 \\
\hline DE0035R & $-0.03(-0.24-0.17)$ & & 0.754 & $-0.10(-0.60-0.39)$ & & 0.683 \\
\hline DE0422A & $0.12(-0.13-0.39)$ & & 0.381 & $0.18(-0.36-0.76)$ & & 0.479 \\
\hline DE0510A & $-0.06(-0.30-0.18)$ & & 0.657 & $-0.09(-0.57-0.45)$ & & 0.690 \\
\hline DE0514A & $-0.01(-0.23-0.21)$ & & 0.921 & $0.03(-0.45-0.52)$ & & 0.877 \\
\hline DE0556A & $0.03(-0.20-0.28)$ & & 0.789 & $0.04(-0.48-0.57)$ & & 0.856 \\
\hline DE0649A & $0.12(-0.12-0.34)$ & & 0.293 & $0.15(-0.35-0.70)$ & & 0.540 \\
\hline
\end{tabular}


Table B3: Quantification of annual CHIMERE model trends (ppbv/yr and \%/yr) in $\mathrm{O}_{3} 95^{\text {th }}$ percentiles 1996-2005. 95\% confidence interval given in brackets. Sig. = significance ${ }^{* * *}$ significant at $99.9 \%$ level, ${ }^{* *}$ significant at $99.0 \%$ level, ${ }^{*}$ significant at $95.0 \%$ level, + significant at $90.0 \%$ level. ${ }^{\ddagger} 2 \sigma$ error. ${ }^{\diamond}$ significant trends only

\begin{tabular}{|c|c|c|c|c|c|c|}
\hline ID & Trend (ppbv/yr) & Sig. & P-value & Trend $(\% / y r)$ & Sig. & P-value \\
\hline$\overline{\mathrm{DE} 0651 \mathrm{~A}}$ & $0.11(-0.12-0.32)$ & & 0.338 & $0.18(-0.28-0.67)$ & & 0.485 \\
\hline DE0674A & $0.05(-0.15-0.31)$ & & 0.615 & $0.12(-0.33-0.68)$ & & 0.650 \\
\hline DE0679A & $-0.02(-0.21-0.19)$ & & 0.789 & $-0.04(-0.43-0.37)$ & & 0.877 \\
\hline DE0680A & $0.11(-0.08-0.34)$ & & 0.235 & $0.30(-0.13-0.78)$ & & 0.190 \\
\hline DE0684A & $0.03(-0.24-0.35)$ & & 0.775 & $-0.06(-0.59-0.47)$ & & 0.772 \\
\hline DE0685A & $0.09(-0.16-0.37)$ & & 0.474 & $0.08(-0.40-0.64)$ & & 0.779 \\
\hline DE0686A & $0.05(-0.17-0.31)$ & & 0.650 & $0.12(-0.37-0.62)$ & & 0.720 \\
\hline DE0687A & $0.05(-0.15-0.31)$ & & 0.615 & $0.12(-0.33-0.68)$ & & 0.650 \\
\hline DE0688A & $0.12(-0.08-0.36)$ & & 0.236 & $0.21(-0.26-0.72)$ & & 0.379 \\
\hline DE0699A & $0.02(-0.26-0.25)$ & & 0.913 & $-0.09(-0.60-0.51)$ & & 0.653 \\
\hline DE0719A & $0.02(-0.20-0.25)$ & & 0.860 & $-0.07(-0.58-0.47)$ & & 0.789 \\
\hline DE0732A & $0.13(-0.08-0.34)$ & & 0.231 & $0.25(-0.18-0.74)$ & & 0.295 \\
\hline DE0735A & $0.12(-0.08-0.36)$ & & 0.236 & $0.21(-0.26-0.72)$ & & 0.379 \\
\hline DE0737A & $0.11(-0.17-0.37)$ & & 0.465 & $0.11(-0.41-0.65)$ & & 0.713 \\
\hline DE0738A & $0.02(-0.19-0.26)$ & & 0.849 & $-0.05(-0.55-0.47)$ & & 0.845 \\
\hline DE0739A & $0.02(-0.24-0.26)$ & & 0.835 & $-0.04(-0.55-0.50)$ & & 0.852 \\
\hline DE0754A & $-0.01(-0.26-0.23)$ & & 0.949 & $-0.02(-0.62-0.51)$ & & 0.928 \\
\hline DE0844A & $-0.16(-0.33-0.02)$ & + & 0.095 & $-0.31(-0.73-0.24)$ & & 0.255 \\
\hline DE0874A & $0.13(-0.05-0.34)$ & & 0.165 & $0.31(-0.14-0.80)$ & & 0.184 \\
\hline DE0907A & $0.14(-0.10-0.36)$ & & 0.222 & $0.19(-0.32-0.75)$ & & 0.479 \\
\hline DE0960A & $-0.08(-0.26-0.10)$ & & 0.350 & $-0.17(-0.65-0.34)$ & & 0.460 \\
\hline DE0996A & $0.08(-0.15-0.30)$ & & 0.490 & $0.17(-0.31-0.67)$ & & 0.479 \\
\hline EE0011R & $-0.15(-0.29-0.02)$ & $*$ & 0.038 & $-0.33(-0.66-0.08)$ & + & 0.065 \\
\hline ES0007R & $0.17(0.00-0.34)$ & + & 0.062 & $0.27(-0.01-0.55)$ & + & 0.067 \\
\hline $\mathrm{ES} 1222 \mathrm{~A}$ & $-0.16(-0.38-0.05)$ & & 0.169 & $-0.35(-0.68-0.05)$ & & 0.102 \\
\hline ES1400A & $0.09(-0.08-0.25)$ & & 0.343 & $0.17(-0.11-0.48)$ & & 0.257 \\
\hline ES1435A & $0.02(-0.11-0.15)$ & & 0.768 & $0.04(-0.20-0.28)$ & & 0.761 \\
\hline ES1437A & $-0.01(-0.13-0.13)$ & & 0.910 & $-0.04(-0.27-0.21)$ & & 0.775 \\
\hline ES1441A & $-0.01(-0.13-0.13)$ & & 0.910 & $-0.04(-0.27-0.21)$ & & 0.775 \\
\hline FR08 & $0.01(-0.25-0.30)$ & & 0.913 & $0.00(-0.51-0.56)$ & & 0.996 \\
\hline GB0002R & $-0.16(-0.36-0.00)$ & $*$ & 0.043 & $-0.36(-0.80-0.02)$ & $*$ & 0.046 \\
\hline GB0006R & $-0.21(-0.35--0.09)$ & $* * *$ & 0.001 & $-0.45(-0.77--0.21)$ & $* * *$ & 0.001 \\
\hline GB0013R & $-0.11(-0.25-0.00)$ & + & 0.051 & $-0.24(-0.57-0.01)$ & + & 0.056 \\
\hline GB0014R & $-0.09(-0.23-0.03)$ & & 0.154 & $-0.32(-0.63--0.04)$ & $*$ & 0.038 \\
\hline GB0015R & $-0.16(-0.33--0.03)$ & $*$ & 0.017 & $-0.38(-0.80--0.04)$ & $*$ & 0.024 \\
\hline GB0031R & $-0.14(-0.31-0.01)$ & $*$ & 0.036 & $-0.30(-0.69-0.08)$ & + & 0.060 \\
\hline GB0033R & $-0.15(-0.37-0.05)$ & & 0.119 & $-0.27(-0.73-0.12)$ & & 0.168 \\
\hline GB0036R & $0.03(-0.14-0.19)$ & & 0.693 & $0.08(-0.29-0.51)$ & & 0.605 \\
\hline GB0037R & $-0.03(-0.20-0.10)$ & & 0.713 & $-0.08(-0.48-0.24)$ & & 0.631 \\
\hline GB0038R & $0.02(-0.13-0.18)$ & & 0.828 & $0.04(-0.28-0.39)$ & & 0.838 \\
\hline GB0039R & $-0.06(-0.18-0.06)$ & & 0.352 & $-0.11(-0.42-0.13)$ & & 0.362 \\
\hline GB0044R & $-0.05(-0.22-0.10)$ & & 0.471 & $-0.14(-0.52-0.22)$ & & 0.394 \\
\hline
\end{tabular}


Table B3: Quantification of annual CHIMERE model trends (ppbv/yr and \%/yr) in $\mathrm{O}_{3} 95^{\text {th }}$ percentiles 1996-2005. 95\% confidence interval given in brackets. Sig. = significance ${ }^{* * *}$ significant at $99.9 \%$ level, ${ }^{* *}$ significant at $99.0 \%$ level, ${ }^{*}$ significant at $95.0 \%$ level, + significant at $90.0 \%$ level. ${ }^{\ddagger} 2 \sigma$ error. ${ }^{\diamond}$ significant trends only

\begin{tabular}{|c|c|c|c|c|c|c|}
\hline ID & Trend (ppbv/yr) & Sig. & P-value & Trend (\%/yr) & Sig. & $\mathrm{P}$-value \\
\hline GB0045R & $0.01(-0.18-0.15)$ & & 0.935 & $-0.02(-0.44-0.32)$ & & 0.910 \\
\hline GB0617A & $0.00(-0.19-0.15)$ & & 0.975 & $-0.02(-0.46-0.38)$ & & 0.899 \\
\hline HPB & $-0.07(-0.28-0.17)$ & & 0.537 & $-0.12(-0.57-0.37)$ & & 0.602 \\
\hline HU0002R & $-0.01(-0.19-0.15)$ & & 0.885 & $-0.05(-0.45-0.33)$ & & 0.806 \\
\hline IE31 & $-0.27(-0.44--0.14)$ & $* * *$ & $<0.001$ & $-0.61(-1.01--0.29)$ & $* * *$ & $<0.001$ \\
\hline IT04 & $-0.27(-0.58-0.07)$ & + & 0.070 & $-0.34(-0.84-0.32)$ & & 0.191 \\
\hline LT0015R & $-0.13(-0.28-0.00)$ & + & 0.054 & $-0.30(-0.62-0.03)$ & + & 0.070 \\
\hline LV0010R & $-0.14(-0.28--0.01)$ & $*$ & 0.035 & $-0.31(-0.61--0.01)$ & $*$ & 0.035 \\
\hline NL0007R & $0.13(-0.09-0.34)$ & & 0.280 & $0.30(-0.23-0.88)$ & & 0.303 \\
\hline NL0009R & $-0.12(-0.29-0.03)$ & & 0.114 & $-0.25(-0.58-0.10)$ & & 0.139 \\
\hline NL0010R & $0.07(-0.14-0.32)$ & & 0.485 & $0.27(-0.19-0.85)$ & & 0.221 \\
\hline NL0196A & $0.10(-0.13-0.36)$ & & 0.386 & $0.26(-0.23-0.87)$ & & 0.305 \\
\hline NL0198A & $0.01(-0.17-0.22)$ & & 0.870 & $0.07(-0.42-0.56)$ & & 0.754 \\
\hline NL0202A & $0.07(-0.14-0.31)$ & & 0.485 & $0.20(-0.29-0.75)$ & & 0.443 \\
\hline NL0205A & $0.05(-0.15-0.28)$ & & 0.653 & $0.11(-0.36-0.63)$ & & 0.663 \\
\hline NL0207A & $-0.03(-0.15-0.09)$ & & 0.611 & $-0.07(-0.35-0.21)$ & & 0.680 \\
\hline NL0209A & $0.03(-0.21-0.26)$ & & 0.814 & $0.03(-0.52-0.67)$ & & 0.899 \\
\hline NL0220A & $-0.03(-0.20-0.12)$ & & 0.586 & $-0.08(-0.44-0.29)$ & & 0.602 \\
\hline NL0223A & $0.10(-0.16-0.36)$ & & 0.414 & $0.26(-0.34-0.87)$ & & 0.364 \\
\hline NL0226A & $-0.04(-0.24-0.17)$ & & 0.683 & $-0.11(-0.57-0.38)$ & & 0.653 \\
\hline NL0227A & $-0.09(-0.24-0.04)$ & & 0.228 & $-0.20(-0.53-0.10)$ & & 0.233 \\
\hline NL0228A & $0.05(-0.15-0.24)$ & & 0.653 & $0.08(-0.30-0.52)$ & & 0.686 \\
\hline NL0229A & $0.03(-0.21-0.26)$ & & 0.814 & $0.03(-0.52-0.67)$ & & 0.899 \\
\hline NL0231A & $0.07(-0.13-0.29)$ & & 0.552 & $0.12(-0.34-0.65)$ & & 0.624 \\
\hline NL0232A & $0.07(-0.18-0.32)$ & & 0.565 & $0.14(-0.44-0.74)$ & & 0.589 \\
\hline NL0250A & $-0.07(-0.22-0.09)$ & & 0.345 & $-0.16(-0.51-0.19)$ & & 0.404 \\
\hline NO01 & $-0.20(-0.36--0.05)$ & $*$ & 0.018 & $-0.46(-0.83--0.09)$ & $*$ & 0.023 \\
\hline PL03 & $-0.08(-0.29-0.14)$ & & 0.457 & $-0.14(-0.62-0.34)$ & & 0.540 \\
\hline PT0004R & $-0.13(-0.37-0.12)$ & & 0.229 & $-0.23(-0.62-0.17)$ & & 0.246 \\
\hline PUY & $-0.03(-0.25-0.16)$ & & 0.772 & $-0.11(-0.50-0.27)$ & & 0.624 \\
\hline SI0008R & $-0.13(-0.33-0.07)$ & & 0.194 & $-0.29(-0.66-0.07)$ & & 0.126 \\
\hline ZUG & $-0.16(-0.33-0.03)$ & + & 0.068 & $-0.24(-0.59-0.16)$ & & 0.199 \\
\hline $\begin{array}{c}\text { European } \\
\text { average }^{\ddagger}\end{array}$ & $-0.03 \pm 0.02 \mathrm{ppbv} / \mathrm{yr}$ & & & $-0.06 \pm 0.04 \% / \mathrm{yr}$ & & \\
\hline Range $^{\diamond}$ & -0.46 to $0.17 \mathrm{ppbv} / \mathrm{yr}$ & & & -3.12 to $2.16 \% / \mathrm{yr}$ & & \\
\hline
\end{tabular}




\section{Sites Used in This Study}

A list of the sites selected for inclusion in this study including their GEOmon recategorisation based on a representiveness study. 
Table C1: Background sites selected from the GEOmon harmonised data set including their categorisation as per Henne et al., 2010

\begin{tabular}{|c|c|c|c|c|c|}
\hline ID & Station & $\begin{array}{l}\text { Longitude } \\
\qquad\left(^{\circ}\right)\end{array}$ & $\begin{array}{l}\text { Latitude } \\
\qquad\left(^{\circ}\right)\end{array}$ & $\begin{array}{l}\text { Altitude } \\
\text { (m a.s.l) }\end{array}$ & $\begin{array}{l}\text { GEOmon } \\
\text { Category }\end{array}$ \\
\hline AT0002R & Illmitz & 16.77 & 47.77 & 117 & rural \\
\hline AT0004R & St. Koloman Kleinhorn & 13.23 & 47.65 & 1005 & rural \\
\hline AT0005R & Vorhegg bei Kötschach-Mauthen & 12.97 & 46.68 & 1020 & valley/basin \\
\hline AT0034R & Sonnblick & 12.97 & 47.05 & 3106 & elevated \\
\hline AT0044A & Streithofen & 15.94 & 48.28 & 220 & rural \\
\hline AT0052A & Piber & 15.08 & 47.08 & 585 & valley/basin \\
\hline AT0054A & Schöneben & 13.95 & 48.71 & 920 & elevated \\
\hline AT0058A & Zillertaler Alpen & 11.87 & 47.14 & 1970 & elevated \\
\hline AT0064A & Innsbruck Nordkette & 11.38 & 47.31 & 1910 & elevated \\
\hline AT0069A & Haunsberg & 13.02 & 47.97 & 730 & elevated \\
\hline AT0073A & Grünbach bei Freistadt & 14.57 & 48.53 & 918 & elevated \\
\hline AT0079A & Sonnblick & 12.96 & 47.05 & 3106 & elevated \\
\hline AT0080A & St. Valentin Stein & 14.56 & 48.23 & 242 & rural \\
\hline AT0086A & Kollmitzberg & 14.87 & 48.18 & 465 & rural \\
\hline AT0089A & Karwendel West & 11.23 & 47.34 & 1730 & elevated \\
\hline AT0094A & Hochgössnitz & 15.02 & 47.06 & 900 & rural \\
\hline AT0095A & Mistelbach & 16.58 & 48.58 & 250 & rural \\
\hline AT0096A & Forsthof am Schöpfl & 15.92 & 48.11 & 581 & rural/polluted \\
\hline AT0101A & Heidenreichstein Thaures & 15.05 & 48.88 & 560 & rural \\
\hline AT0102A & Wolkersdorf & 16.52 & 48.39 & 190 & rural/polluted \\
\hline AT0103A & Stixneusiedl & 16.68 & 48.05 & 210 & rural/polluted \\
\hline AT0105A & Irnfritz & 15.5 & 48.72 & 556 & rural \\
\hline AT0108A & Masenberg & 15.88 & 47.35 & 1137 & elevated \\
\hline AT0111A & Dunkelsteinerwald & 15.55 & 48.37 & 305 & rural/agricultural \\
\hline AT0115A & Grundlsee & 13.8 & 47.62 & 980 & rural \\
\hline AT0121A & Höfen Lächbichl & 10.68 & 47.47 & 880 & valley/basin \\
\hline AT0122A & Kramsach Angerberg & 11.91 & 47.46 & 600 & valley/basin \\
\hline AT0124A & Gerlitzen Steinturm & 13.90 & 46.68 & 1895 & elevated \\
\hline AT0128A & $\begin{array}{c}\text { St. Georgen im Lavanttal - } \\
\text { Herzogberg }\end{array}$ & 14.89 & 46.71 & 540 & rural \\
\hline AT0134A & Annaberg - Joachimsberg & 15.32 & 47.86 & 891 & rural \\
\hline AT0141A & Obervellach Schulzentrum & 13.2 & 46.94 & 686 & valley/basin \\
\hline AT0143A & Bleiburg Koschatstrasse & 14.8 & 46.59 & 480 & rural \\
\hline AT0146A & Stolzalpe bei Murau & 14.2 & 47.13 & 1302 & rural \\
\hline AT0149A & Pillersdorf bei Retz & 15.94 & 48.72 & 315 & rural \\
\hline AT0153A & Arnfels - Remschnigg & 15.37 & 46.65 & 785 & elevated \\
\hline AT0154A & Wiesmath & 16.29 & 47.61 & 738 & elevated \\
\hline AT0162A & Liezen & 14.24 & 47.57 & 665 & valley/basin \\
\hline AT0164A & Oberwart - Brunnenfeld & 16.19 & 47.3 & 330 & rural \\
\hline AT0166A & Zell am See Krankenhaus & 12.81 & 47.34 & 770 & valley/basin \\
\hline AT0167A & Payerbach & 15.85 & 47.67 & 890 & rural \\
\hline AT0175A & Klöch bei Bad Radkersburg & 15.96 & 46.75 & 300 & rural \\
\hline AT0176A & $\begin{array}{l}\text { Zöbelboden - } \\
\text { Reichraminger Hintergebirge }\end{array}$ & 14.44 & 47.84 & 899 & elevated \\
\hline AT0180A & Hochwurzen & 13.63 & 47.36 & 1850 & elevated \\
\hline BE0032R & Eupen & 6 & 50.63 & 295 & rural/polluted \\
\hline
\end{tabular}


Table C1: continued. Background sites selected from the GEOmon harmonised data set including their categorisation as per Henne et al., 2010

\begin{tabular}{|c|c|c|c|c|c|}
\hline ID & Station & $\begin{array}{l}\text { Longitude } \\
\left(^{(}\right)\end{array}$ & $\begin{array}{l}\text { Latitude } \\
\quad\left({ }^{\circ}\right)\end{array}$ & $\begin{array}{l}\text { Altitude } \\
\text { (m a.s.l) }\end{array}$ & $\begin{array}{l}\text { GEOmon } \\
\text { Category }\end{array}$ \\
\hline BE0033R & Moerkerke & 3.36 & 51.26 & 3 & rural/coastal \\
\hline BE0035R & Vezin & 4.99 & 50.5 & 160 & rural/polluted \\
\hline BE0211A & Gellik & 5.62 & 50.88 & 70 & suburban \\
\hline BE0238A & Offagne & 5.2 & 49.88 & 430 & rural \\
\hline BE0294A & Walshoutem & 5.1 & 50.71 & 135 & rural/polluted \\
\hline BE0298A & Idegem & 3.93 & 50.8 & 15 & rural/polluted \\
\hline BE0302A & Habay-la-n & 5.63 & 49.72 & 375 & rural \\
\hline BE0304A & Dourbes & 4.59 & 50.1 & 225 & rural \\
\hline BE0311A & Saint-ode & 5.59 & 50.03 & 510 & rural \\
\hline BE0345A & St.P.Leeuwg & 4.23 & 50.77 & 20 & suburban \\
\hline CH0001R & Jungfraujoch & 7.98 & 46.55 & 3573 & elevated \\
\hline $\mathrm{CH} 0002 \mathrm{R}$ & Payerne & 6.94 & 46.81 & 489 & rural \\
\hline CH0003R & Tänikon & 8.9 & 47.48 & 539 & rural/polluted \\
\hline CH0004R & Chaumont & 6.98 & 47.05 & 1137 & elevated \\
\hline CH0005R & Rigi-Seebodenalp & 8.46 & 47.07 & 1031 & elevated \\
\hline CH0019A & St. Gallen Stuelegg & 9.39 & 47.41 & 920 & elevated \\
\hline CH0024A & Saxon & 7.15 & 46.14 & 460 & valley/basin \\
\hline CH0033A & Magadino-Cadenazzo & 8.93 & 46.16 & 204 & valley/basin \\
\hline CMN & Monte Cimone & 10.68 & 44.17 & 2165 & elevated \\
\hline CZ0001R & Svratouch & 16.04 & 49.74 & 735 & elevated \\
\hline CZ0003R & Kosetice & 15.08 & 49.57 & 535 & rural \\
\hline CZ0017A & Bily Kriz & 18.54 & 49.5 & 890 & elevated \\
\hline CZ0030A & Sous & 15.32 & 50.79 & 771 & elevated \\
\hline CZ0041A & Ondrejov & 14.78 & 49.92 & 514 & rural/polluted \\
\hline CZ0045A & Kostelni Myslova & 15.44 & 49.16 & 569 & rural \\
\hline CZ0049A & Churanov & 13.61 & 49.07 & 1118 & elevated \\
\hline CZ0051A & Jesenik & 17.19 & 50.24 & 625 & elevated \\
\hline CZ0055A & Krkonose-Rychory & 15.85 & 50.66 & 1001 & elevated \\
\hline CZ0057A & Hojna Voda & 14.72 & 48.72 & 818 & elevated \\
\hline CZ0062A & Rudolice v Horach & 13.42 & 50.58 & 840 & elevated \\
\hline DE0003R & Schauinsland & 7.91 & 47.91 & 1205 & elevated \\
\hline DE0007R & Neuglobsow & 13.03 & 53.14 & 65 & rural \\
\hline DE0008R & Schmücke & 10.77 & 50.65 & 937 & elevated \\
\hline DE0009R & Zingst & 12.72 & 54.44 & 1 & rural/coastal \\
\hline DE0035R & Lückendorf & 14.79 & 50.83 & 490 & rural \\
\hline DE0422A & Riedstadt & 8.52 & 49.83 & 87 & rural/polluted \\
\hline DE0510A & Neustadt a.d. Donau/Eining & 11.78 & 48.85 & 359 & rural \\
\hline DE0514A & Mehring/Sportplatz & 12.78 & 48.18 & 415 & rural \\
\hline DE0556A & Zinnwald & 13.75 & 50.73 & 877 & elevated \\
\hline DE0649A & Grebenau & 9.46 & 50.76 & 373 & rural \\
\hline DE0651A & Witzenhausen/Wald & 9.77 & 51.29 & 610 & elevated \\
\hline DE0674A & Simmerath Eifel & 6.28 & 50.65 & 572 & elevated \\
\hline DE0679A & Tiefenbach/Altenschneeberg & 12.55 & 49.44 & 755 & elevated \\
\hline DE0680A & Horn-Bad Meinberg Egge & 8.95 & 51.83 & 430 & elevated \\
\hline DE0684A & Schwarzwald Süd & 7.76 & 47.81 & 920 & elevated \\
\hline DE0685A & Westpfalz-Waldmohr & 7.29 & 49.42 & 455 & elevated \\
\hline
\end{tabular}


Table C1: continued. Background sites selected from the GEOmon harmonised data set including their categorisation as per Henne et al., 2010

\begin{tabular}{|c|c|c|c|c|c|}
\hline ID & Station & $\begin{array}{c}\text { Longitude } \\
\left(^{\circ}\right)\end{array}$ & $\begin{array}{l}\text { Latitude } \\
\quad\left({ }^{\circ}\right)\end{array}$ & $\begin{array}{l}\text { Altitude } \\
\text { (m a.s.l) }\end{array}$ & $\begin{array}{l}\text { GEOmon } \\
\text { Category }\end{array}$ \\
\hline DE0686A & Hunsrück-Leisel & 7.19 & 49.74 & 650 & elevated \\
\hline DE0687A & Westeifel Wascheid & 6.38 & 50.27 & 680 & elevated \\
\hline DE0688A & Westerwald-Herdorf & 7.97 & 50.77 & 480 & elevated \\
\hline DE0699A & Welzheimer Wald & 9.57 & 48.88 & 500 & rural/polluted \\
\hline DE0719A & Spessart & 9.4 & 50.16 & 502 & rural/polluted \\
\hline DE0732A & Solling & 9.58 & 51.76 & 500 & elevated \\
\hline DE0735A & Netphen Rothaargebirge & 8.19 & 50.93 & 635 & elevated \\
\hline DE0737A & Pfälzerwald-Hortenkopf & 7.83 & 49.27 & 606 & elevated \\
\hline DE0738A & Naila/Selbitzer Berg & 11.72 & 50.32 & 534 & rural \\
\hline DE0739A & Fürth/Odenwald & 8.82 & 49.65 & 484 & rural/polluted \\
\hline DE0754A & B Grunewald (3.5 m) & 13.23 & 52.47 & 50 & suburban \\
\hline DE0844A & Bornhöved & 10.24 & 54.09 & 45 & rural/polluted \\
\hline DE0874A & Soest-Ost & 8.15 & 51.57 & 110 & rural \\
\hline DE0907A & Nidda & 9 & 50.42 & 193 & rural/polluted \\
\hline DE0960A & Ueckermünde & 14.07 & 53.74 & 1 & rural/coastal \\
\hline DE0996A & Wurmberg & 10.61 & 51.76 & 930 & elevated \\
\hline EE0011R & Vilsandi & 21.85 & 58.38 & 6 & remote \\
\hline ES0007R & Viznar & -3.32 & 37.14 & 1265 & elevated \\
\hline ES1222A & Santa Maria de Palautordera & 2.44 & 41.69 & 208 & rural/polluted \\
\hline ES1400A & Bujaraloz & -0.15 & 41.51 & 325 & - \\
\hline ES1435A & Vilafranca & -0.25 & 40.43 & 1125 & rural \\
\hline ES1437A & Coratxar & 0.08 & 40.69 & 1200 & rural \\
\hline ES1441A & Morella & -0.09 & 40.64 & 1150 & rural \\
\hline FR08 & Donon & 7.13 & 48.5 & 775 & elevated \\
\hline GB0002R & Eskdalemuir & -3.21 & 55.32 & 269 & rural \\
\hline GB0006R & Lough Navar & -7.9 & 54.44 & 130 & rural \\
\hline GB0013R & Yarner Wood & -3.72 & 50.6 & 119 & rural \\
\hline GB0014R & High Muffles & -0.81 & 54.33 & 267 & rural \\
\hline GB0015R & Strath Vaich & -4.78 & 57.73 & 270 & remote \\
\hline GB0031R & Aston Hill & -3.33 & 52.5 & 370 & rural \\
\hline GB0033R & Bush Estate & -3.21 & 55.86 & 180 & suburban \\
\hline GB0036R & Harwell & -1.33 & 51.57 & 137 & rural \\
\hline GB0037R & Lady Bower & -1.75 & 53.4 & 420 & rural/polluted \\
\hline GB0038R & Lullington Heath & 0.18 & 50.79 & 125 & rural/coastal \\
\hline GB0039R & Sibton & 1.46 & 52.29 & 46 & rural/coastal \\
\hline GB0044R & Somerton & -2.74 & 51.04 & 55 & rural \\
\hline GB0045R & Wicken Fen & -0.29 & 52.3 & 5 & rural/polluted \\
\hline GB0617A & Rochester Stoke & 0.63 & 51.46 & 14 & $\mathrm{rural} /$ polluted \\
\hline HPB & Hohenpeissenberg & 11.02 & 47.8 & 985 & elevated \\
\hline HU0002R & K-puszta & 19.55 & 46.97 & 125 & rural/agricultural \\
\hline IE31 & Mace Head & -9.9 & 53.33 & 25 & remote \\
\hline IT04 & Ispra & 8.63 & 45.8 & 209 & valley/basin \\
\hline LT0015R & Preila & 21.03 & 55.38 & 5 & rural/coastal \\
\hline LV0010R & Rucava & 21.17 & 56.16 & 18 & rural \\
\hline NL0007R & Eibergen-Lintveldseweg & 6.61 & 52.09 & 19 & rural \\
\hline NL0009R & Kollumerwaard-Hooge Zuidwal & 6.28 & 53.33 & 1 & rural/coastal \\
\hline
\end{tabular}


Table C1: continued. Background sites selected from the GEOmon harmonised data set including their categorisation as per Henne et al., 2010

\begin{tabular}{cccccc} 
ID & Station & $\begin{array}{c}\text { Longitude } \\
\left({ }^{\circ}\right)\end{array}$ & $\begin{array}{c}\text { Latitude } \\
\left({ }^{\circ}\right)\end{array}$ & $\begin{array}{c}\text { Altitude } \\
(\mathrm{m} \text { a.s.l })\end{array}$ & $\begin{array}{c}\text { GEOmon } \\
\text { Category }\end{array}$ \\
\hline NL0010R & Vredepeel-Vredeweg & 5.85 & 51.54 & 28 & rural/polluted \\
NL0196A & Budel-Toom & 5.56 & 51.27 & 32 & rural/polluted \\
NL0198A & Zierikzee-Lange Slikweg & 3.92 & 51.64 & -1 & rural/coastal \\
NL0202A & Posterholt-Vlodropperweg & 6.04 & 51.12 & 32 & rural/polluted \\
NL0205A & Hellendoorn-Luttenbergerweg & 6.4 & 52.39 & 7 & rural \\
NL0207A & Balk-Trophornsterweg & 5.57 & 52.92 & 0 & rural/coastal \\
NL0209A & Cabauw-Zijdeweg & 4.93 & 51.97 & -1 & rural/polluted \\
NL0220A & Philippine-Stelleweg & 3.75 & 51.3 & 5 & rural/coastal \\
NL0223A & Biest Houtakker-Biestsestraat & 5.15 & 51.52 & 15 & suburban \\
NL0226A & Westmaas-Groeneweg & 4.45 & 51.79 & 0 & rural/polluted \\
NL0227A & Wieringerwerf- & 5.05 & 52.8 & -4 & rural/coastal \\
& Medemblikkerweg & & & & \\
NL0228A & Biddinghuizen-Hoekwantweg & 5.62 & 52.45 & -4 & rural/coastal \\
NL0229A & Zegveld-Oude Meije & 4.84 & 52.14 & -2 & rural/polluted \\
NL0231A & Barsbeek-De Veenen & 6.02 & 52.66 & 0 & rural \\
NL0232A & Huijbergen-Vennekenstraat & 4.36 & 51.44 & 18 & suburban \\
NL0250A & De Zilk-Vogelaarsdreef & 4.51 & 52.3 & 4 & rural/polluted \\
NO01 & Birkenes & 8.25 & 58.38 & 190 & rural \\
PL03 & Sniezka & 15.74 & 50.74 & 1603 & elevated \\
PT0004R & Monte Velho & -8.8 & 38.08 & 43 & rural/coastal \\
PUY & Puy de Dome & 3 & 45.75 & 1465 & elevated \\
SI0008R & Iskrba & 14.86 & 45.56 & 540 & rural/agricultural \\
ZUG & Zugspitze & 10.98 & 47.42 & 2950 & elevated \\
\hline
\end{tabular}

